\title{
Gualou Guizhi Granule Suppresses LPS-induced Inflammatory Response of Microglia and Protects Against Microglial-Mediated Neurotoxicity in HT22 via Akt/NF-kB Signaling Pathways
}

\section{Yuqin Zhang}

Fujian University of Traditional Chinese Medicine

Yaojun Liu

Fujian University of Traditional Chinese Medicine

\section{Liming Fan}

Fujian University of Traditional Chinese Medicine Lihong Nan

Fujian University of Traditional Chinese Medicine

Wei Xu

Fujian University of Traditional Chinese Medicine

\section{Kedan Chu}

Fujian University of Traditional Chinese Medicine

\section{Guohong Yan}

Fujian University of Traditional Chinese Medicine

Yu Lin ( $\nabla$ lyfjlab@163.com)

Fujian University of Traditional Chinese Medicine https://orcid.org/0000-0001-5033-3733

\section{Research}

Keywords: Gualou Guizhi Granule, microglia, LPS, neuroinflammation, Akt, NF-kB

Posted Date: November 18th, 2020

DOl: https://doi.org/10.21203/rs.3.rs-104142/v1

License: (c) (i) This work is licensed under a Creative Commons Attribution 4.0 International License. Read Full License 


\section{Abstract}

Background Neuroinflammation plays a crucial part in the commence and advancement of ischemic stroke. Gualou Guizhi Granule (GLGZG) is known to exhibit well neuroprotective effect, but it is not known whether GLGZG can regulate inflammatory process at the cellular level in BV2 microglia cells and protect against microglial-mediated neurotoxicity in neurons. Herein, we aimed to investigate the antiinflammatory effects of GLGZG on BV2 microglia cells and protection against microglial-mediated neurotoxicity in neurons.

Methods Cell model of neuroinflammation was constructed by lipopolysaccharide (LPS) to observe the effect of GLGZG in the presence or absence of GLGZG. The production of nitric oxide (NO), inflammatory mediators were detected. Moreover, potential mechanisms associated with antiinflammatory effect, such as inhibition of microglial activation, the nuclear factor kappa B (NF-KB) were also investigated. In addition, to prove whether GLGZG protects against microglial-mediated neurotoxicity, neuronal HT22 cells were cultured in conditioned medium. And cell survivability, neuronal apoptosis of HT22 were evaluated.

Results It was found that a main regulator of inflammation, NO, is suppressed by GLGZG in BV2 microglial cells. Moreover, GLGZG dose-dependently decreased the mRNA and protein levels of inducible NO synthase (iNOS) in LPS-stimulated BV2 cells. Additionally, GLGZG inhibited the expression and secretion of proinflammatory cytokines in BV2 microglial cells. And, GLGZG inhibited LPS-activated nuclear factor kappa-light-chain-enhancer of activated B cells (NF-kB) in BV2 microglial cells at the intracellular level. GLGZG negatively affected Akt phosphorylation: phosphorylated forms of Akt decreased. To check whether GLGZG protects against microglial-mediated neurotoxicity, neuronal HT22 cells were incubated in the conditioned medium. GLGZG showed a neuroprotective effect by promoting cell survivability, suppressing neuronal apoptosis.

Conclusions GLGZG exerted its potential effects on suppressing inflammatory responses in LPS-induced BV2 cells by attenuating NF-KB and Akt pathways. In addition, GLGZG could protect against microglialmediated neurotoxicity in $\mathrm{HT} 22$.

\section{Background}

Stroke, especially ischemic stroke, is a life-threatening disease which is considered the most common cause of disability and mortality around the world [1,2]. Although ischemic stroke involve the interaction of numerous pathophysiological processes, accumulating evidence proves that neuroinflammation plays an important role in the pathological process involved in ischemic stroke [3-6]. Recent studies proved have proved that microglia is critically involved in regulating neuroinflammation [7].

Microglia as the main immune cells in the brain, act as the first major safeguard of the central nervous system (CNS). In physiological state, microglia are at the resting state, they survey the CNS microenvironment via continuously extending and retracting ramified processes; while they were 
activated in a number of neurodegenerative diseases and different types of brain injury [8-10]. Ischemic stroke is a powerful stimulus triggers microglial activation. Once activated, microglia develop macrophage-like capabilities, and polarize into M1 or M2 phenotype. They are considered as a doubleedged sword [11, 12]. M2 microglia are regarded as "healing cells", they clear debris, release antiinflammatory mediators and function to help repair injury. On the other hand, M1 microglia are considered as proinflammatory, releasing pro-inflammatory mediators, and function to exacerbate ischemic injury, at the same time, peripheral leukocytes infiltrate into the brain and the normally immuneprivileged brain environment is exposed to systemic responses that further exacerbate inflammation and brain damage, resulting in a vicious cycle. Thus, regulating microglial phenotype might be a potential treatment for neuroinflammation of ischemic stroke.

It is proved that the activated microglia-mediated neuroinflammation is mainly regulated by transcription factor nuclear factor kappa B (NF-KB), a crucial modulator of various kinds of inflammation [13]. As we know, NF-kB is bound to the inhibitor of kappaB (IкB) proteins in the cytosol which is inactive. And when the IKB kinase (IKK) firstly phosphorylates and degrades IKB, NF-KB begin to be activated, NF-KB is dissociated from complex and transfers to the nucleus. Then, triggering the generation of proinflammatory molecules. What's more, the phosphatidylinositol 3-kinase/Akt (PI3K/Akt) signal pathway is also involved in NF-KB activation and pro-inflammatory responses [14].

Chinese classical prescription are important resources to develop safe and effective candidates for neuroinflammation of ischemic stroke. Gualou Guizhi granule (GLGZG, Min drug system approval No.S20130001), is a standard hospital prescription at Fujian University of TCM Affiliated Second People's Hospital (Fuzhou, China), which is first reported by Zhang Zhongjing in Jin Kui Yao Lue [15]. It consists of 6 kinds of Chinese herbs: Trichosanthes kirilowii Maxim., Cinnamomum cassia Presl., Paeoniae lactiflora Pall., Zingiber officinale Rosc., Ziziphus jujuba Mill. and Glycyrrhiza uralensis Fisch. It has long been used in clinic to treat muscular spasticity and dyskinesia following stroke, epilepsy or spinal cord injury in China with a weight ratio of 10:3:3:3:2:3 [16-21].

Moreover, in recent years, studies have also documented that GLGZG exerts well effects of antiinflammation, anti cerebral ischemia reperfusion injury and neuroprotection [22-25]. Our group have shown that GLGZG exhibited significant anti-inflammatory effects to reduce inflammatory cytokines, chemokines and mediators, such as tumor necrosis factor (TNFa), interleukin (IL)1 $\beta$, IL6, monocyte chemotactic protein 1 (MCP1) and nitric oxide (NO) in vivo[26]. Although preliminary researches suggested a potential relationship between GLGZG and neuroinflammation, whether GLGZG can regulate neuroinflammation, and the underlying mechanisms, have not been well investigated. In the current study, we aimed to investigate the anti-neuroinflammatory effects of GLGZG in LPS-stimulated BV-2 microglial cells. Furthermore, the roles of GLGZG in Akt/NF-KB related signaling pathway were investigated.

\section{Methods}

\section{Reagents and Chemicals}


GLGZG was presented by Pharmaceutical Department of Fujian University of Traditional Chinese Medicine Affiliated Second People's Hospital (Fuzhou, China). It was certified and standardized on the basis of labeled compounds (The Food and Drug Administration in Fujian Province 2013). Our phytochemical studies also illustrated that 104 compounds in GLGZG were identified or tentatively characterized and several bioactive components, such as citrulline, luteolin, puerarin, liquiritin, taxifolin, naringin, formononetin, isoliquiritigenin, 6-gingerol, curcumin, caffeic acid, ferulic acid, jujuboside $A$, protocatechuic acid, cinnamic acid, catechin, paeoniflorin [22,29-31]. Lipopolysaccharide (LPS) was purchased from Sigma (St. Louis, USA). RPMI Medium 1640 basic and fetal bovine serum (FBS) were purchased from Thermo Fisher Scientific (Carlsbad, CA, USA). CellTiter 96® AQ ueous one solution cell proliferation assay was bought from Promega (Madison, USA). IL-1 $\beta$, IL-6, IL-10 and TNF-a mouse ELISA kits were obtained from (ABclonal (Wuhan) biotechnology co. LTD, Wuhan, China). Rabbit antibodies against Akt, p-Akt(Ser473), PI3K(p85), PI3K(p110a), IKK 3 , p-ІKBa, IKBa, NF-KBp65 and $\beta$-actin were purchased from Cell Signaling Technology (Boston, MA, USA). The secondary antibodies conjugated with horseradish peroxidase (HRP) were all bought from Xiamen LuLong Biotech Co., Ltd. (Xiamen, China).

\section{Cell Culture And Treatment}

BV-2 microglial cells and neuronal HT22 were obtained from Beijing Beina Chuanglian Biotechnology Research Institute (Beijing, China). In brief, cells were cultured in RPMI 1640 medium with $10 \%$ FBS and $1 \%$ penicillin-streptomycin mixed solution (Gibco Invitrogen Corporation, Carlsbad, CA, USA) at $37^{\circ} \mathrm{C}$ in a $5 \% \mathrm{CO}_{2}$ incubator.

In these studies, the cells were seeded on 96-well plates or 6-well plates and were divided into three groups randomly: (1) pure BV2 cell group as control group (BV2 group); (2) LPS group, where BV2 cells were incubated with LPS $(1 \mu \mathrm{g} / \mathrm{mL})$ for $24 \mathrm{~h}$; and (3) LPS plus GLGZG $(50,100 \rrbracket 200 \mu \mathrm{g} / \mathrm{mL})$ group, where cells were co-incubated with LPS $(1 \mu \mathrm{g} / \mathrm{mL})$ and $\operatorname{GLGZG~}(50,100,200 \mu \mathrm{g} / \mathrm{mL})$ for $24 \mathrm{~h}$. Culture supernatants were harvested for ELISA experiments and nitric oxide assay. Additionally, the cells were then harvested for RNA or protein isolation.

To determine protective effect of GLGZG against microglial-mediated neurotoxicity, neuronal HT22 were incubated in conditioned medium from the control, LPS, and LPS plus GLGZG groups of BV2 cells. Conditioned media were then tested for cell viability using CellTiter $96{ }^{\circledR}$ AQueous one solution cell proliferation and LDH assays, and cell lysates were used to test for apoptosis from conditioned mediatreated HT-22 cells.

\section{Cell Viability Assay}

After treatment, the cell viability was assessed by CellTiter $96 \AA$ AQueous one solution cell proliferation assay. CellTiter $96 \AA$ AQueous one solution was added and incubated for another $4 \mathrm{~h}$ at $37^{\circ} \mathrm{C}$. Then the 
absorbance at $570 \mathrm{~nm}$ was taken by a microplate reader (Infinite M200 Pro, TECAN). The cell viability was calculated according to the $O D$ value. Survival rate $(\%)=O D_{\text {experimental group }} / O D_{\text {control group }} \times 100 \%$ ).

\section{Elisa Experiments}

After treatment, culture supernatants were collected and then centrifuged prior to the determination of IL$1 \beta$, IL-6, IL-10 and TNF-a production. Detailed manipulation process was performed by manufacturer protocols of mouse ELISA kits.

\section{Nitric Oxide Assay}

After treatment, the culture supernatants were collected, and NO production was measured by assessing the nitrite level in the culture media. It was executed by mixing the medium with griess reagent. Optical concentration was analyzed at $540 \mathrm{~nm}$ after 10 minutes incubation.

\section{Quantitative Real-time Pcr Analysis}

After treatment, cells were washed with PBS, and the total RNA was extracted by RNeasy® Mini Kit, and the RNA concentration were determined. Then Revert Aid First strand cDNA Synthsis Kit was used to reverse transcription to CDNA. The CDNA product was used as a template for quantitative PCR amplification, and it was then carried out on ABI 7900HT real-time PCR system (Applied Biosystems, Inc., Foster City, CA, USA). The data were analyzed by $2^{-\triangle \triangle C T}$ relative quantification method. The relative transcriptional level of target genes normalized to GAPDH was calculated. The primer sequences for amplification of the target genes are shown in Table 1. The relative transcriptional level of target genes normalized to GAPDH was calculated. 
Table 1

Primers used for quantitative real-time PCR analysis

\begin{tabular}{|lll|}
\hline Gene & Forward primer & Reverse primer \\
\hline CD32 & 5'-AATCCTGCCGTTCCTACTGATC-3' & 5'-GTGTCACCGTGTCTTCCTTGAG-3' \\
\hline CD86 & 5'-GACCGTTGTGTGTGTTCTGG-3' & 5'-GATGAGCAGCATCACAAGGA - 3' \\
\hline CD206 & 5'-CAAGGAAGGTTGGCATTTGT-3' & 5'-CCTTTCAGTCCTTTGCAAGC-3' \\
\hline Arginase1 & 5'-TCACCTGAGCTTTGATGTCG-3' & 5'-CTGAAAGGAGCCCTGTCTTG-3' \\
\hline Ym1 & 5'-CAGGGTAATGAGTGGGTTGG-3' & 5'-CACGGCACCTCCTAAATTGT-3' \\
\hline IL-13 & 5'-ATG ACC TGT TCT TTG AGG CTG AC- & 5'-CGA GAT GCT GCT GTG AGA TTT G-3' \\
\hline IL-6 & 5'-GAC CAA GAC CAT CCA ACT CAT C-3' & 5'-ACA TTC ATA TTG CCA GTT CTT CGT A- \\
\hline IL-10 & 5'-CCAAGCCTTATCGGAAATGA-3' & 5'- TTTTCACAGGGGAGAAATCG-3' \\
\hline TNF-a & 5'-ATG AGC ACG GAA AGC ATG-3' & 5'-TAC GGG CTT GTC ACT CGA GTT-3' \\
\hline iNOS & 5'-CAA GCA CCT TGG AAG AGG AG-3' & 5'-AAG GCC AAA CAC AGC ATA CC-3' \\
\hline GAPDH & 5'-AGC CCA GAA CAT CAT CCC TG-3' & 5'- AGC CCA GAA CAT CAT CCC TG-3' \\
\hline
\end{tabular}

\section{Western Blot Analysis}

Western Blot Analysis

The cells were inoculated into 6-well plate with a density of $3 \times 10^{5} /$ well and cultured for $24 \mathrm{~h}$, then treated with indicated concentrations of GLGZG and LPS $(1 \mu \mathrm{g} / \mathrm{mL})$ for $24 \mathrm{~h}$. Cells were collected and lysed by lysis buffer, then they were centrifuged at $12,000 \mathrm{~g}$ for $15 \mathrm{~min}$. The supernatant was collected and the protein concentration was determined by the BCA method. Then protein mixed with loading buffer and incubated in $100{ }^{\circ} \mathrm{C}$ for $6 \mathrm{~min}$. Ultimately, samples were analyzed for western blot analysis with primary antibodies to iNOS(1:1000), CD206(1:1000), p-AKT(Ser473)(1:2000), AKT(1:1000), PI3K(p85)(1:1000), PI3K(p110a)(1:1000), IKKß(1:1000), p-IKBa(1:1000), IKBa(1:1000), NF-KBp65(1:1000), $\operatorname{Bax}(1: 1000), \mathrm{Bcl}-2(1: 1000), \mathrm{NeuN}(1: 1000)$ and $\beta$-actin(1:1000) overnight at $4{ }^{\circ} \mathrm{C}$ followed by incubating with the horseradish peroxidase-conjugated secondary antibody IgG (HRP Goat Anti-Rabbit IgG secondary antibody (1:5000), Goat Anti-Mous IgG secondary antibody (1:5000), LuLong Biotech Co., Xiamen, China) at room temperature. Finally they were evaluated using the ECL western detection reagents and the relative expression level of target genes normalized to $\beta$-actin was analyzed.

\section{Immunofluorescence Assay}


Immunofluorescence assay was performed to detect the nuclear translocation of NF-kBp65 subunit. In brief, cells were fixed using $4 \%$ paraformaldehyde for $15 \mathrm{~min}$ at room temperature, followed by washing with PBS for 5 min 3 times. The cells were blocked with 5\% BSA prepared in $0.1 \%$ Tween 20 (PBST) and $0.3 \%$ Triton $\mathrm{X}-100$ for $1 \mathrm{~h}$ at room temperature. Afterward, cells were incubated with NF-kBp65 specific primary antibody $\left(1: 100\right.$, Cell Signaling Technology, MA, USA) overnight at $4{ }^{\circ} \mathrm{C}$. After washing, cells were incubated with FITC-labeled IgG secondary antibody (Beijing Zhongshan Jinqiao Biotechnology Co. LTD, Beijing, China, 1:200 dilution in 5\% BSA solution) for $1 \mathrm{~h}$ in dark. After that, cells were stained with $25 \mu \mathrm{g} / \mathrm{mL}$ of 4'-6-diamidino-2-phenylindole (DAPI) in PBST. Finally, samples were documented using Olympus IX73 fluorescence microscope (Tokyo, Japan).

\section{Statistical analysis}

All the experiments were repeated three times. The data were statistically analyzed by SPSS 19.0 software and expressed as mean \pm standard deviation ( $x \pm s$ ). The difference between groups was analyzed by one-way ANOVA followed by the Student-Newman-Keuls q test with post-hoc correction was employed for the multiple comparisons, and $P<0.05$ was considered to indicate statistical significance.

\section{Results}

\section{GLGZG modulate M1/M2 microglial polarization in the LPS- induced activation of microglia}

According to the past research result, 50,100 and $200 \mu \mathrm{g} / \mathrm{mL}$ of GLGZG were employed in these experiments [26]. As illustrated in Fig. 1, GLGZG in the presence and absence of LPS did not have a significant effect on microglial viability.

Morphological examination result is presented in representative photomicrographs in Fig. 2. As shown in Fig. 2, the LPS-activated cells were characterized by round and an absence of thin processes, which was considered as an "active stage" phenotype. While, as compared to LPS-activated cells, the control and GLGZG-treated LPS-activated BV2 cells showed less rounded and more elongated cells with thinner processes, which is considered as "resting stage" phenotype.

To further confirm these findings, expression of M1 and M2 markers of BV2 in culture were determinated by real-time PCR. As shown in Fig. 3A-3C, LPS treatment caused a significantly increase in mRNA levels of three M1 markers: iNOS $(P<0.01), \mathrm{CD} 32(P<0.01)$ and CD86 $(P<0.05)$, and GLGZG treatment obviously attenuated the effect for iNOS $(P<0.05, P<0.01), \operatorname{CD} 32(P<0.05, P<0.01)$ and CD86 $(P<0.05$, $P<0.01)$. Western blot analysis of the M1 marker-iNOS (Fig. 3D-3E), it was indicates that the protein express of iNOS is significantly up-regulated after LPS activation of BV2 microglia cells $(P<0.01$, Fig. 3D$3 \mathrm{E})$, while this effect is obviously attenuated by GLGZG treatment $(P<0.05)$. Likewise, mRNA expression of three M2 markers and the protein express of the M2 marker-CD206 were examinated. As shown in Fig. 4, LPS treatment have no significant effect on CD206, Arginase1 and Ym1, three M2 markers, 
however GLGZG treatment significantly elevated the expression for CD206, Arginase1 and Ym1 $(P<0.05$, $P<0.01)$. Protein result of CD206 revealed that LPS had no significant effect on CD206 protein, while GLGZG treatment significantly up-regulated CD206 protein.

\section{Glgzg Suppressed Lps-induced Inflammatory Response In Lps-activated Bv-2 Microglial}

To further investigate the effect of GLGZG on neuroinflammation in LPS-activated BV-2 microglial, we determined the effect of GLGZG on neuro-inflammatory responses, such as cytokines including IL-1 $\beta$, IL-6, IL-10, MCP-1 and TNF-a, inflammatory mediators including NO. The effect of GLGZG on LPS induced microglia release IL-1 $\beta$, IL-6, IL-10, MCP-1, TNF- $\alpha$ and NO were shown in Fig. 5. Compared with the control group, the content of IL-1 $\beta$, IL-6, MCP-1, TNF- $\alpha$ and NO in the LPS group increased significantly, while compared with the LPS group, IL-1 $\beta$, IL-6, MCP-1, TNF- $\alpha$ and NO were decreased significantly in the cells of the 50,100 and $200 \mu \mathrm{g} / \mathrm{mL}$ dose group of GLGZG. In addition, GLGZG also markedly increased the production of IL-10.

Moreover, the mRNA expression of IL-1 $\beta$, IL-6, TNF- $\alpha$ and iNOS were measured, all of which were inhibited by treatment with GLGZG (Fig. 6). These results suggest that GLGZG not only inhibits LPS induced NO production, iNOS, IL-1 $\beta$, IL-6, IL-10, TNF-a, and also significantly reduces mRNA expression of them.

\subsection{GLGZG inhibites activation of the NF-KB pathway in LPS-activated BV-2 microglial}

As shown in Fig. 7A-E, GLGZG dose-dependently suppressed NF-KBp65 expression in the nuclear compared with the average levels in LPS group $(P<0.01)$. And to confirm the reduced nuclear translocation, regulators of NF-KB in the cytosol, such as IKB, p-ІkB and IkK $\beta$, were investigated. As expected, IKBa expression decreased and increased with GLGZG treatment, while $\mathrm{p}-\mathrm{I} \mathrm{KBa}$ and IkK $\beta$ expression respectively increased and decreased with GLGZG treatment compared to LPS group $(P<$ $0.01)$.

Furthermore, in order to check the decrease in translocation of the nuclear transcription factor, we directly assayed the intracellular translocation of the factor by immunofluorescence, and NF-kB nuclear translocation in LPS-treated cells reduced with GLGZG treatment, as observed by immunofluorescence microscopic analysis, indicating specific suppression of NF-KB nuclear translocation (Fig. 8).

\section{Glgzg Suppresses Akt Phosphorylation In Lps-activated Bv- 2 Microglial}

As shown in Fig. 9, GLGZG could significantly increase the expression of p-AKT(Ser473) and PI3K(p85) compared with LPS group. While, the expression of AKT and PI3K(p110a) had no significant change among groups. 


\section{Glgzg Protect Against Microglial-mediated Neurotoxicity}

As shown in Fig. 10A, the results show that conditioned medium from LPS plus GLGZG-treated BV2 microglia cells significantly enhanced cell viability $(69.56 \%, 72.13 \%, 86.34 \%)$ on HT-22 cells, as compared to the LPS-treated BV2 microglia cell conditioned medium $(P<0.01)$. Furthermore, as shown in Fig. 10B-E, the levels of Bcl-2 notably declined, while cleaved caspase-3 and Bax increased in LPS-treated BV2 microglia cell conditioned medium. While GLGZG exhibited a more pronounced effect on Bcl-2, cleaved caspase-3 and Bax expression.

\section{Discussion}

Neuroinflammatory responses is inevitable and vital pathological processes in ischemic stroke, and microglial activation-mediated inflammatory responses play an important role in brain neuroinflammation and subsequent neuronal injuries. Activated microglia can be characterized by M1 (proinflammatory) and M2 (neuroprotective/anti-inflammatory) phenotype [32]. BV2 cells stimulated by LPS could provide a very reproducible and robust model for induction of M1 phenotype and an inflammatory activation profile of BV2 microglia cells, and it is highly similar to activation of primary microglia [33,34]. Since GLGZG was found to be anti-inflammatory and neuroprotective in several reports, in this study, whether GLGZG could act directly upon microglia cells to regulate microglial polarization, pro- and anti-inflammatory cytokine gene expression, and the neurotoxic ability of activated microglia, were evaluated in vitro.

Indeed, the results revealed that GLGZG decreased production of the M1 markers, iNOS, CD32, TNF- $\mathrm{a}$ and IL-1 $\beta$, while increased production of the M2 marker, CD206, arginase1 and Ym1. Correspondingly, GLGZG also obviously inhibited the production of the pro-inflammatory mediators IL-1 $\beta$, IL-4, IL-6, TNF-a and IFNy and increased the level of IL-10 in LPS-activated BV2 microglia cells. As we know that, pro-inflammatory cytokines, e.g. TNF-a, IL-6, and IL-1 $\beta$, can promote neuronal damage, and also induce more microglia activation as feedback [35]. In addition, some studies indicated that enhanced M2 polarization may be beneficial due, in large part, to a switch of production from M1 "proinflammatory cytokines" to M2 "antiinflammatory cytokines" thus decreasing inflammation and facilitating tissue and cellular repair [36, 37]. The findings suggested that GLGZG exerted anti-neuroinflammatory effects by inhibiting BV2 activation.

Moreover, at the intracellular level, GLGZG was also shown to inhibit LPS-activated nuclear translocation of NF-KB in BV2 cells. To our knowledge, NF-KB signaling pathway is the most common involved pathway in the inflammatory response $[38,39]$. NF-KBp65, one of the NF-KB subunits, is inactivated at normal physiological conditions and is bound to the inhibitor of kappaB (ІкB) proteins in the cytosol. While when it is stimulated by inflammatory stimuli, such as viruses, bacterial toxins LPS, IKK firstly phosphorylates and degrades IKB, NF-KB begin to be activated, NF-KB is dissociated from complex and transfers to the nucleus. In this study, we found that GLGZG markedly inhibited LPS-induced NF-KB activation in BV2 cells by decreasing the phosphorylation level of IKBa, NF-KB p65 nuclear level and IKK induced by LPS stimulation. Furthermore, it is proved that the classical NF-KB pathway mediates not only microglial 
activation, but also neuron death [36]. Our previous experiment found that GLGZG produces its protective effect by inhibiting the apoptosis of neurons. And in this report, our data support that GLGZG suppressed microglial activation via inhibiting NF-KB signal pathways.

$\mathrm{PI3K} /$ Akt pathway, a classical signaling pathway, is involved in NF-KB-mediated neuroinflammation. Activation of Akt take part in regulating pro-inflammatory responses in microglia by modulating NF-KB signal pathways [40]. As expected, herein, we found that GLGZG activates the phosphorylation of Akt induced by LPS in BV-2 microglia. Therefore, these results suggested that GLGZG might specifically suppresse neuroinflammatory responses and the activation of NF-KB signaling pathways in activated BV2 cells via activation of the Akt signal pathways.

Activated microglia release $\mathrm{NO}$ and proinflammatory cytokines, induce neuroinflammation, which are known to damage neurons [41]. Next, to examine whether GLGZG treatment protected against activated microglia-mediated neuroinflammation. As shown in results (Fig. 9A), GLGZG significantly improved cell viability in LPS treatment-induced HT22 cells and reduced neuron apoptosis. Thus, these results suggest that GLGZG inhibition of microglial activation could prevent injury to neurons.

\section{Conclusions}

To conclude, findings from this study illuminated that GLGZG could attenuate the inflammatory response of LPS-activated microglia via NF-KB and Akt signaling pathway, and protect neurons against activated microglia-mediated neuroinflammation. The study provides evidence that GLGZG may be a potential treatment for neuroinflammation of ischemic stroke.

\section{Abbreviations}

GLGZG, Gualou Guizhi Granule; LPS, lipopolysaccharide; iNOS, inducible NO synthase; NF-kB, nuclear factor kappa B; IKB, inhibitor of kappaB; IKK, IKB kinase; TNFa, tumor necrosis factor, IL, interleukin; MCP1, monocyte chemotactic protein 1; NO, nitric oxide.

\section{Declarations}

\section{Ethics approval and consent to participate}

Not applicable

\section{Consent for publication}

Not applicable

\section{Availability of data and materials}


The datasets used and/or analysed during the current study are available from the corresponding author on reasonable request.

\section{Competing interests}

The authors declare that they have no conflict of interest.

\section{Funding Statement}

This study was funded by the National Natural Science Foundation of China (No. 81803768, No.81674046, No. 81873031), Research platform opening project of fujian university of traditional Chinese medicine (No. X2017017-Pingtai), Fujian Provincial Department of Education to Support Provincial University Special Project (No. JK2017023) and Fujian Provincial Health and Family Planning Commission Youth Talent Project (No. 2017-1-17).

\section{Author Contributions}

ZYQ conceived the study, designed the experiment, carried out the real-time PCR, ELISA and Western blotting measurement; and drafted the manuscript. LYJ and NLH mainly cultured cells and performed the Western blot analysis, the ELISA test and analyzed the data. FLM did the measurement of real-time PCR. CKD and XW contributed to the statistical analysis and manuscript editing. YGH and LY contributed to the research design and coordination, secured funding for the project, helped to draft the manuscript, and took responsibility for the work. All authors read, discussed, and approved the final manuscript.

\section{Acknowledgements}

Not applicable

\section{References}

1. Go AS, D Mozaffarian, VL Roger, et al (2014) American Heart Association Statistics Committee and Stroke Statistics Subcommittee, Heart disease and stroke statistics-2014 update: a report from the American Heart Association. Circulation 129: e28-e292.

2. GBD 2013 Mortality and Causes of Death Collaborators (2015) Global, regional, andnational agesex specific all-cause and cause-specific mortality for 240 causes of death, 1990-2013: asystematic analysis for the Global Burden of Disease Study 2013. Lancet 385: 117-171.

3. Zhang F, W Yin, J Chen (2004) Apoptosis in cerebral ischemia: executional and regulatory signaling mechanisms. J Neurological Res 26: 835-845.

4. Broughton BR, DC Reutens, CG Sobey (2009) Apoptotic mechanisms after cerebral ischemia. Stroke 40: e331-e339.

5. Eltzschig HK, T Eckle (2011) Ischemia and reperfusion-from mechanism to translation. Nat Med 17: 1391-1401. 
6. Wang J, P Wang, S Li, et al (2014) Mdivi-1 prevents apoptosis induced by ischemia-reperfusion injury in primary hippocampal cells via inhibition of reactive oxygen species-activated mitochondrial pathway. J Stroke Cerebrovasc Dis 23(6): 1491-1499.

7. Xu L, D He, Y Bai (2016) Microglia-mediated inflammation and neurodegenerative disease. J Mol Neurobiol 53(10): 6709-6715.

8. Neumann H, MR Kotter, RJM Franklin (2009) Debris clearance by microglia: an essential link between degeneration and regeneration. Brain 132: 288-295.

9. Nimmerjahn A, F Kirchhoff, F Helmchen (2005) Neuroscience: resting microglial cells are highly dynamic surveillants of brain parenchyma in vivo. Sci 308: 1314-1318.

10. Eyo UB, ME Dailey (2013) Microglia: key elements in neural development, plasticity, and pathology. J Neuroimmune Pharmacol 8: 494-509.

11. Patel AR, R Ritzel, LD McCullough, et al (2013) Microglia and ischemic stroke: a double-edged sword. Int J Physiol Pathophysiol Pharmacol 5: 73-90.

12. Wood $P$ (1995) Microglia as a unique cellular target in the treatment of stroke: potential neurotoxic mediators produced by activated microglia. Neurol Res 17: 242-248.

13. Lee Y, JK Yun, MH Kim, et al (2016) MAPK cascades in guard cell signal transduction. Front Plant Sci 7: 80.

14. Saponaro C, A Cianciulli, R Calvello, et al (2012) The PI3K/Akt pathway is required for LPS activation of microglial cells. Immunopharmacol Immunotoxicol 34(5): 858-865.

15. Zhang Z In: Jinkui Yaolue, Y Lin, P Yang, X Hou, Y Yang (ed) (2008) Synopsis of Golden Chamber. Macmillan Press, Beijing, pp 203-204.

16. Zhang L, H Ai (2005) Effects of Gua Lou Gui Zhi decoction on c-fos and c-jun in epileptic rats. Sichuan Huaxi Zhong Yi YaoYan Jiu Suo 23: 21-22.

17. Sun $X$ (2010) Research on formula treating paralysis and spasticity from 'treatise on febrile and miscellaneous diseases'. Zhongguo Zhong Yi Ji Chu Yi Xue Za Zhi 8: 644-645.

18. Yang C, L Chen, J Tao (2010) New usage of a classical formula-Gua Lou Gui Zhi decoction. Liaoning Zhong Yi Za Zhi 8: 166-167.

19. Chen Y, L Chen, J Tao (2013) Clinical research on treating limbs spasm from cerebral apoplexy with the Gualou Guizhi decoction. CJCM. 5(4): 7-9.

20. Zhu Y (2015) Clinical effect of analysis of Trichosanthes Kirilowii Cassia Twig Decotion for treatment of lower limb spasm after cerebral apoplexy patients. Chin J Med Guide 13(29): 27-28.

21. Lin L, X Cheng, $Z$ Lin, et al (2018) Therapeutic Observation of Acupoint lontophoresis with Gualou Guizhi Decoction for Spastic Foot Drop After Cerebral Stroke. Shanghai J Acu-mox 37(07): 746-750.

22. 22 Chen X, H Li, M Huang, et al (2014) Effect of Gua Lou Gui Zhi decoction on focal cerebral ischemia-reperfusion injury through regulating the expression of excitatory amino acids and their receptors. Mol Med Rep 10(1): 248-254. 
23. Lin $\mathrm{Y}, \mathrm{W}$ Xu, W Xu, et al (2015) Simultaneous determination of 41 components in Gualou Guizhi granules by UPLC coupled with triple quadrupole mass spectrometry. Anal Methods 7: 8285-8829.

24. Zhang YQ, H Li, M Huang, et al (2015) Neuroprotective effects of Gualou Guizhi Decoction in vivo and in vitro. J Ethnopharmacol 158: 76-84.

25. Zhang YQ, S Zhang, H Li, et al (2015) Ameliorative effects of Gualou Guizhi Decoction on inflammation in focal cerebral ischemic-reperfusion injury. Mol Med Rep 12: 988-999.

26. Hu H, Z Li, X Zhu, et al (2013) Gua Lou Gui Zhi decoction suppresses LPS-induced activation of the TLR4/NF-KB pathway in BV-2 murine microglial cells. Int J Mol Med 31: 1327-1332.

27. Hu H, X Zhu, Z Li, et al (2015) Anti-inflammatory effect of Gua Lou Gui Zhi decoction on activated microglia. Practical Clinical Journal of Integrated Traditional Chinese and Western Medicine. 17 (1): 152-155.

28. Orihuela R, CA McPherson, GL Harry (2016) Microglial M1/M2 polarization and metabolic states. British J Pharmacol 173(4): 649-665.

29. Xu W, MQ Huang, H Li, et al (2015) Chemical profiling and quantification of Gua-Lou-Gui-Zhi decoction by high performance liquid chromatography/quadrupole-time-of-flight mass spectrometry and ultra-performance liquid chromatography/triple quadrupole mass spectrometry. J Chromatogr B 986-987: 69-84.

30. Lin Y, W Xu, YQ Zhang (2015) Anti-apoptosis effects of Gualou Guizhi granule on stroke rats and hippocampal neuron. Rehabilitation Medicine 25: 38-43.

31. Sun CT, W Xu, YQ Zhang et al (2017). Simultaneous Determination of 11 Compounds in Gualou Guizhi Granule and Pharmacokinetics Study by UPLC-MS/MS. Journal of Analytical Methods in Chemistry. https//doi.org:/10.1155/2017/8451383.

32. Henn AS Lund, M Hedtjärn, A Schrattenholz, et al (2009) The suitability of BV2 cells as alternative model system for primary microglia cultures or for animal experiments examining brain inflammation. Altex-alternativen Zu Tierexperimenten 26(2): 83-94.

33. Thakkar R, R Wang, J Wang, et al (2018) $17 \beta$-Estradiol Regulates Microglia Activation and Polarization in the Hippocampus Following Global Cerebral Ischemia. Oxidative Medicine and Cellular Longevity. https//doi.org:/10.1155/2018/4248526.

34. Block ML, L Zecca, JS Hong (2007) Microglia-mediated neurotoxicity: uncovering the molecular mechanisms. Nature Reviews Neuroscience 8: 57-69.

35. Subramaniam SR, HJ Federoff (2017) Targeting microglial activation states as a therapeutic avenue in Parkinson's disease. Frontiers in Aging Neuroscience 9: 176.

36. Du L, Y Zhang, Y Chen, et al (2017) Role of microglia in neurological disorders and their potentials as a therapeutic target. Molecular Neurobiology 54(10): 7567-7584.

37. Hoesel B, JA Schmid (2013) The complexity of NF- $K$ B signaling in inflammation and cancer. Molecular Cancer 12: 86. 
38. Bak MJ, SG Hong, JW Lee, et al (2012) Red ginseng marc oil inhibits iNOS and COX-2 via NF $k B$ and p38 pathways in LPS-stimulated RAW264.7 macrophages. Molecules 17: 13769-13786.

39. Frakes AE, L Ferraiuolo, AM Haidet-Phillips, et al (2013) Microglia induce motor neuron death via the classical NF-kappaB pathway in amyotrophic lateral sclerosis. Neuron 81(5): 1009-1023.

40. Jiang $Y, X$ Fan, $Y$ Wang, et al (2015) Hepato-protective effects of six schisandra lignans on acetaminophen-induced liver injury are partially associated with the inhibition of CYP-mediated bioactivation. Chemico-Biological Intearctions 231: 83-89.

41. Boje KM, PK Arora (1992) Microglial-produced nitric oxide and reactive nitrogen oxides mediate neuronal cell death. Brain Research 587(2): 250-256.

\section{Figures}

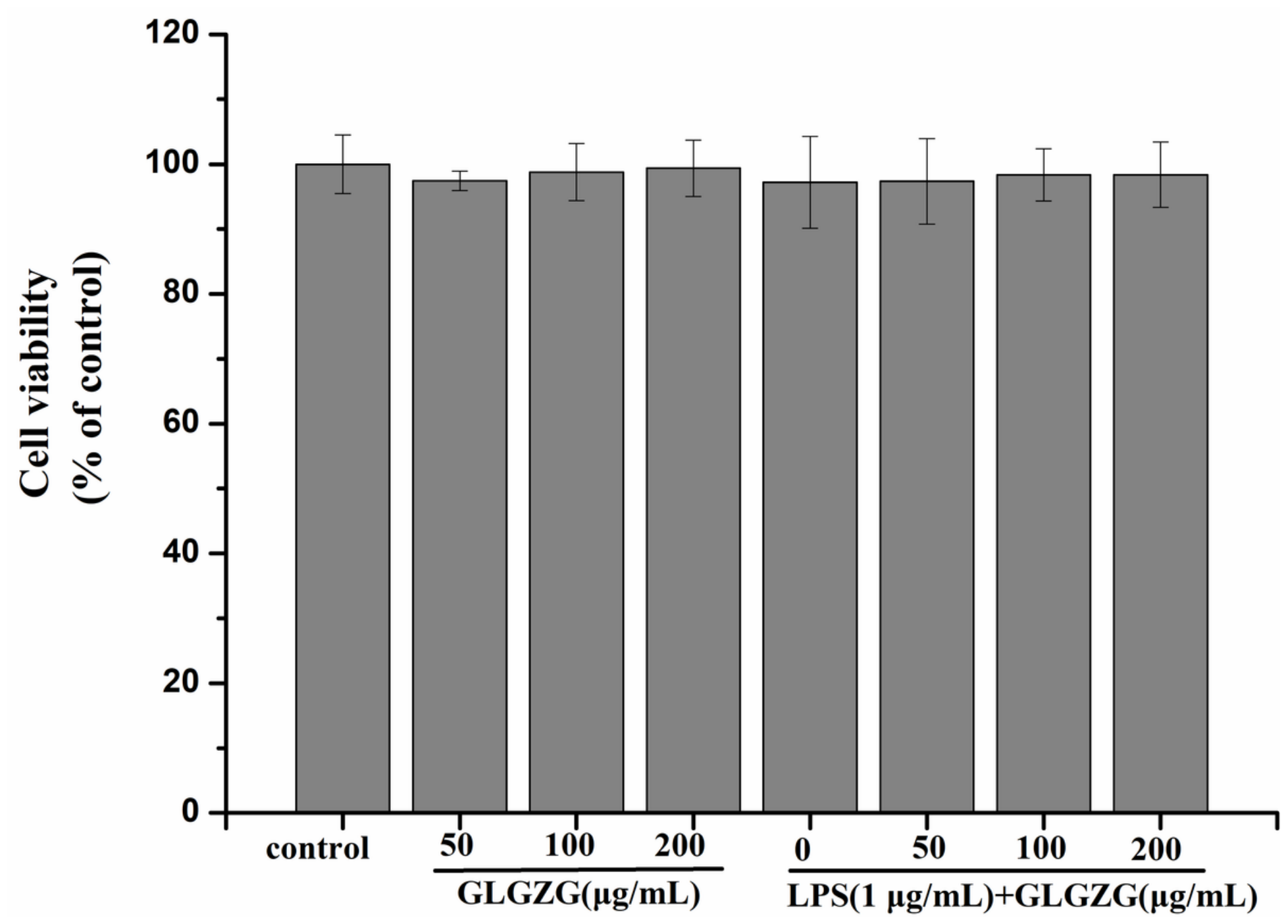

Figure 1

Effects of Gualou Guizhi Granule (GLGZG) on the cell viability of BV-2 microglial cells with or without LPS stimulation. Cells were treated with 50,100 and $200 \mu \mathrm{g} / \mathrm{mL}$ of GLGZG and incubated in the presence and 
absence of $1 \mu \mathrm{g} / \mathrm{mL}$ LPS for $24 \mathrm{~h}$. Cell viability was determined by CellTiter $96 \AA$ AQ ueous one solution. Results were expressed as percentages relative to the control group. Data were shown as means \pm SD of six independent experiments in triplicate.

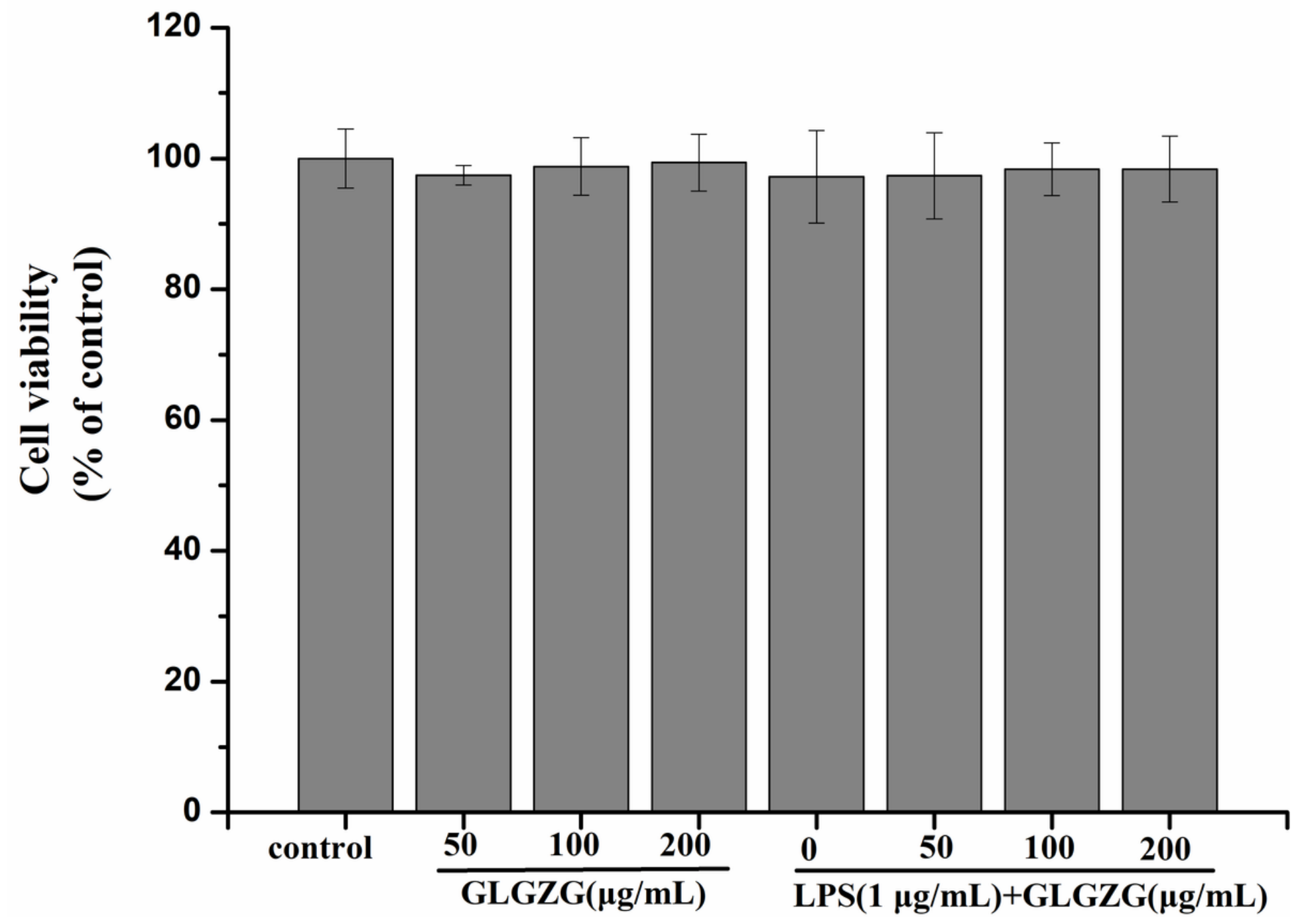

Figure 1

Effects of Gualou Guizhi Granule (GLGZG) on the cell viability of BV-2 microglial cells with or without LPS stimulation. Cells were treated with 50,100 and $200 \mu \mathrm{g} / \mathrm{mL}$ of GLGZG and incubated in the presence and absence of $1 \mu \mathrm{g} / \mathrm{mL}$ LPS for $24 \mathrm{~h}$. Cell viability was determined by CellTiter $96 \AA$ AQ ueous one solution. Results were expressed as percentages relative to the control group. Data were shown as means \pm SD of six independent experiments in triplicate. 


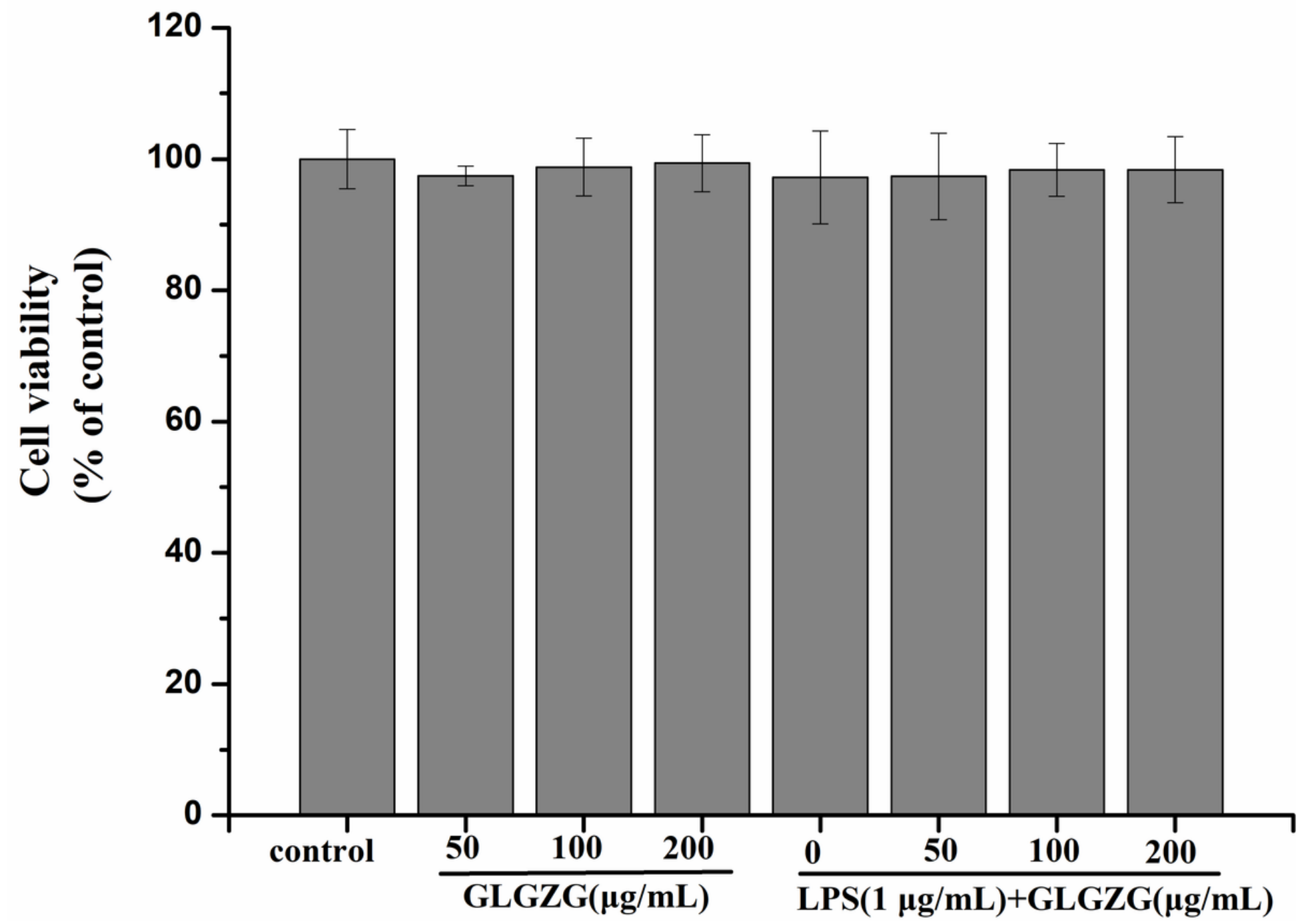

Figure 1

Effects of Gualou Guizhi Granule (GLGZG) on the cell viability of BV-2 microglial cells with or without LPS stimulation. Cells were treated with 50,100 and $200 \mu \mathrm{g} / \mathrm{mL}$ of GLGZG and incubated in the presence and absence of $1 \mu \mathrm{g} / \mathrm{mL}$ LPS for $24 \mathrm{~h}$. Cell viability was determined by CellTiter $96 \AA$ AQ ueous one solution. Results were expressed as percentages relative to the control group. Data were shown as means \pm SD of six independent experiments in triplicate. 


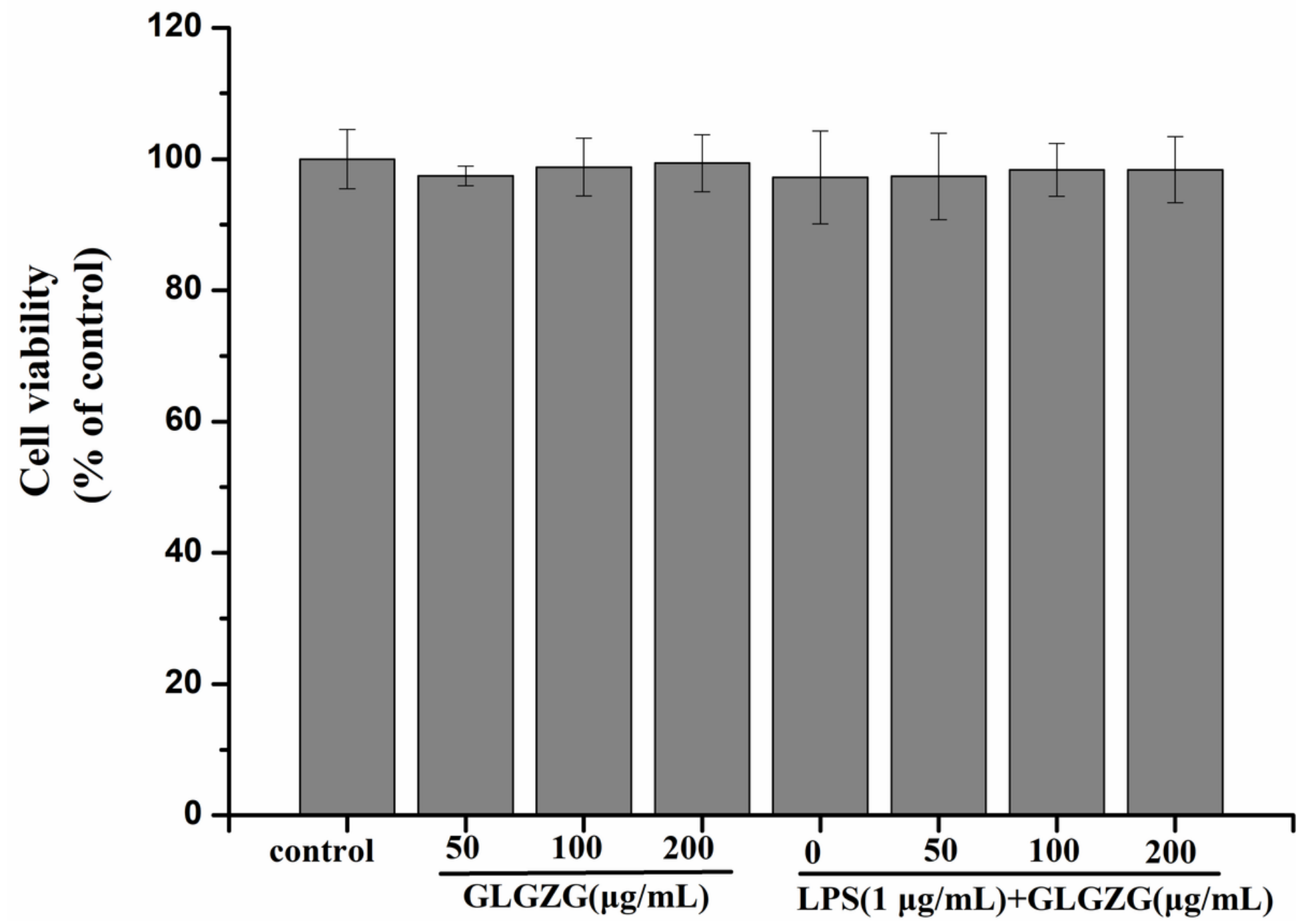

Figure 1

Effects of Gualou Guizhi Granule (GLGZG) on the cell viability of BV-2 microglial cells with or without LPS stimulation. Cells were treated with 50,100 and $200 \mu \mathrm{g} / \mathrm{mL}$ of GLGZG and incubated in the presence and absence of $1 \mu \mathrm{g} / \mathrm{mL}$ LPS for $24 \mathrm{~h}$. Cell viability was determined by CellTiter $96 \AA$ AQ ueous one solution. Results were expressed as percentages relative to the control group. Data were shown as means \pm SD of six independent experiments in triplicate. 


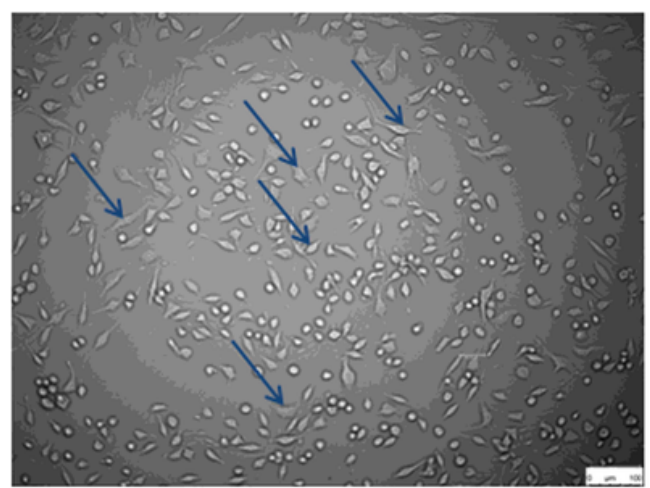

control

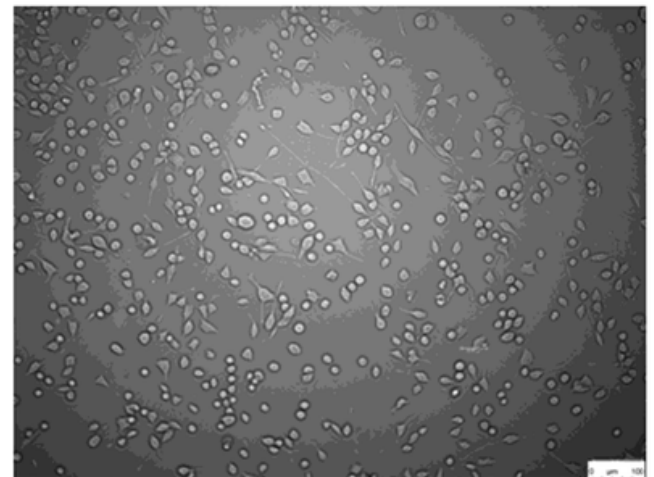

GLGZG $50 \mu g / m L$

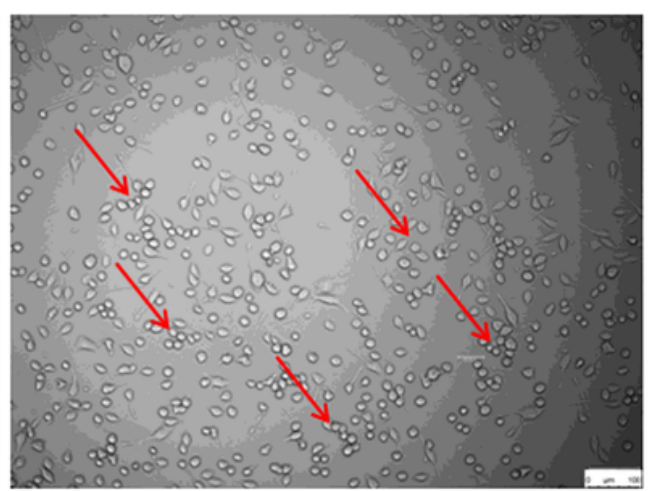

LPS

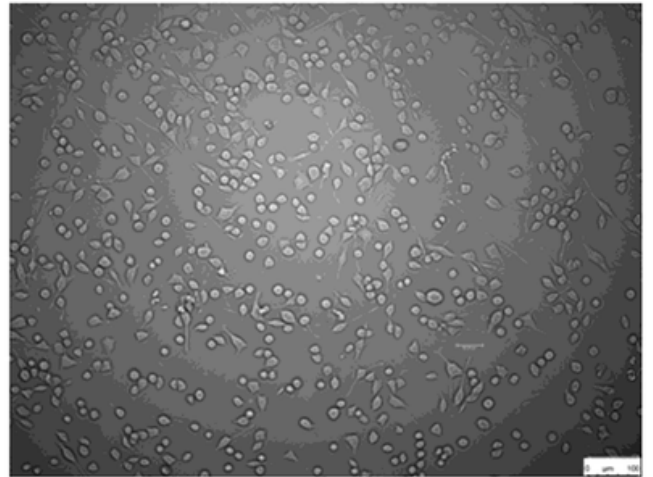

GLGZG $100 \mu \mathrm{g} / \mathrm{mL}$

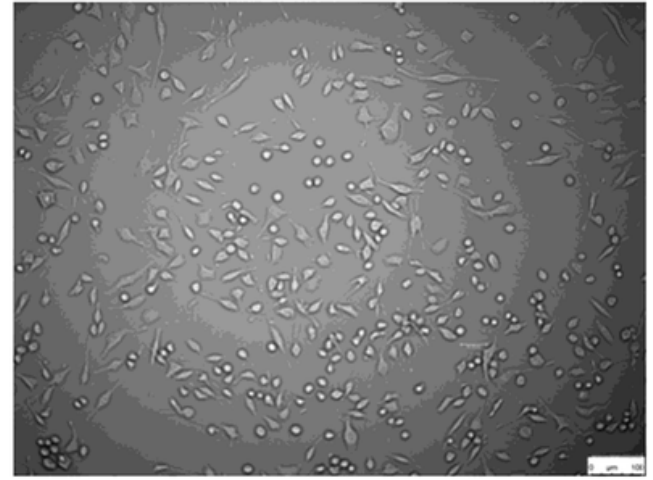

GLGZG $200 \mu \mathrm{g} / \mathrm{mL}$

Figure 2

Effects of Gualou Guizhi Granule (GLGZG) on the morphological characteristics with or without LPS stimulation. Representative bright field microscopy images show the morphological differences among nonactivated control, LPS-activated and activated + GLGZG (50, 100 and $200 \mu \mathrm{g} / \mathrm{mL})$-treated BV2 microglia cells. 


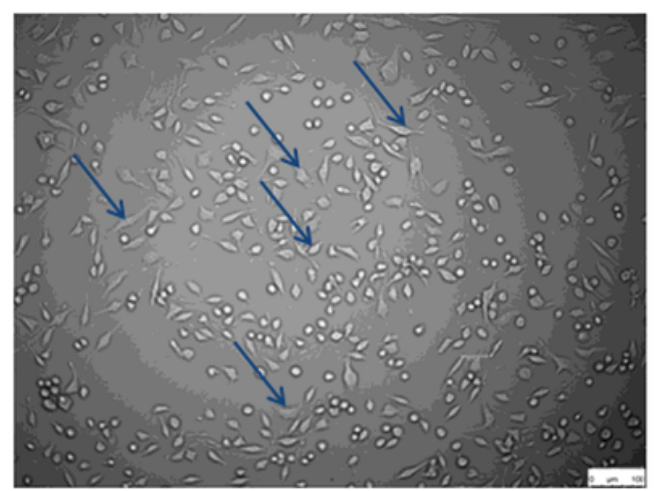

control

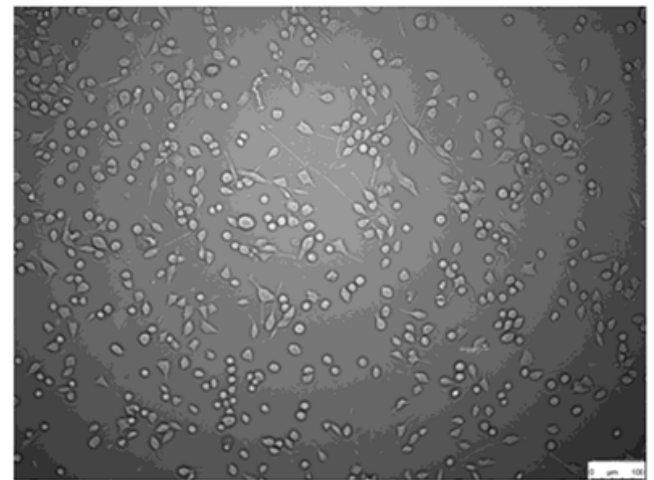

GLGZG $50 \mu \mathrm{g} / \mathrm{mL}$

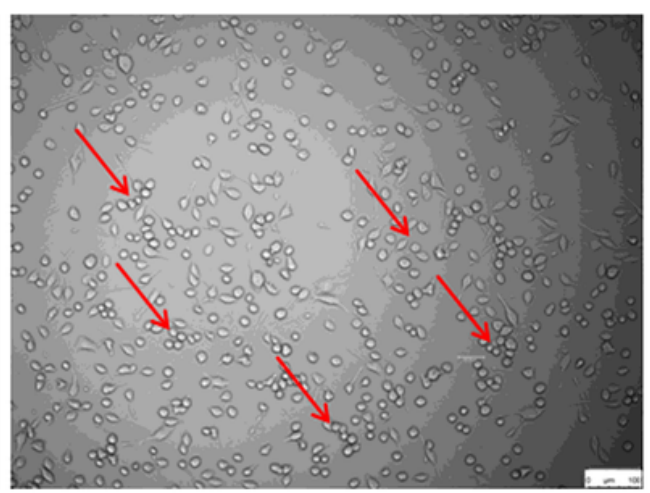

LPS

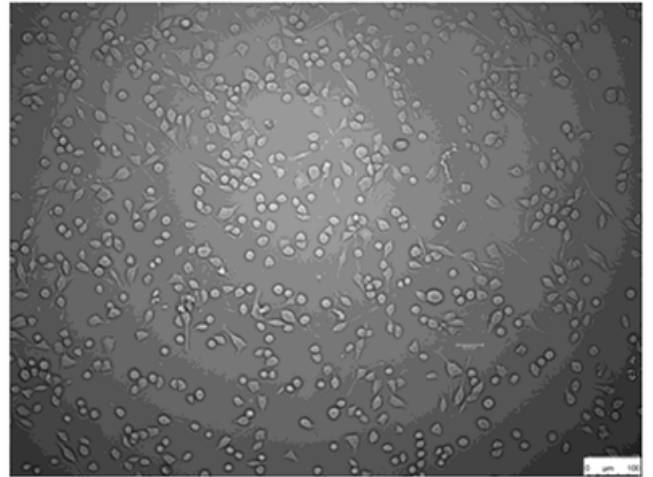

GLGZG $100 \mu \mathrm{g} / \mathrm{mL}$

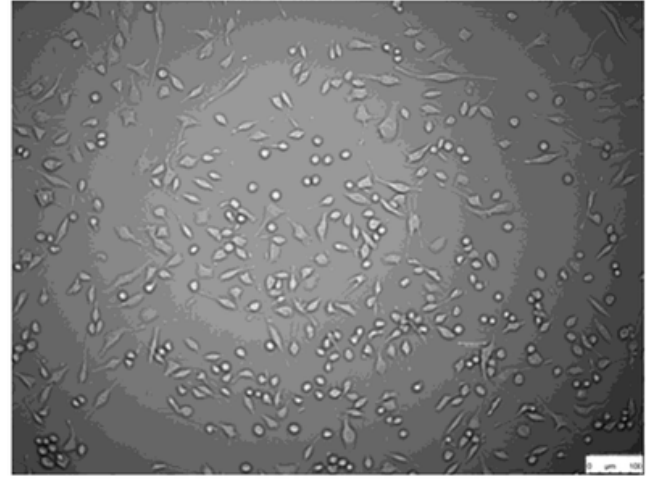

GLGZG $200 \mu \mathrm{g} / \mathrm{mL}$

Figure 2

Effects of Gualou Guizhi Granule (GLGZG) on the morphological characteristics with or without LPS stimulation. Representative bright field microscopy images show the morphological differences among nonactivated control, LPS-activated and activated + GLGZG (50, 100 and $200 \mu \mathrm{g} / \mathrm{mL})$-treated BV2 microglia cells. 


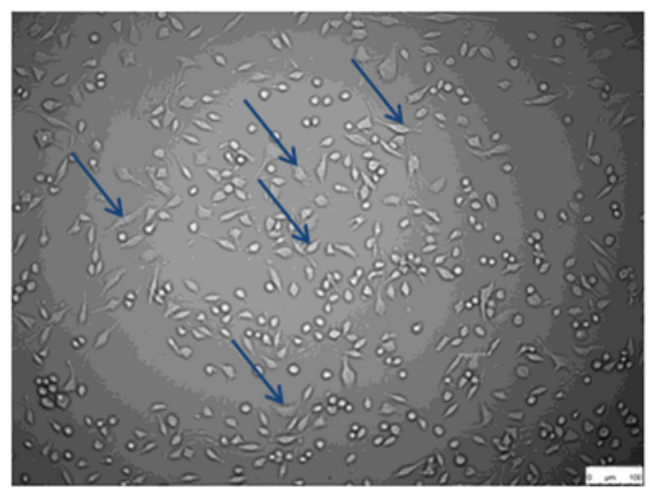

control

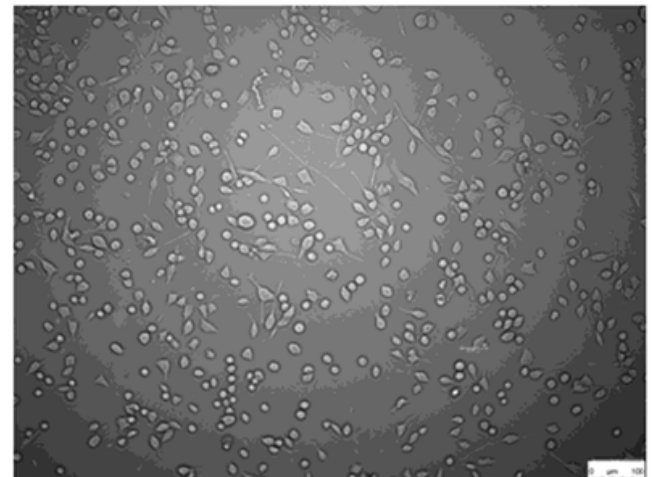

GLGZG $50 \mu g / m L$

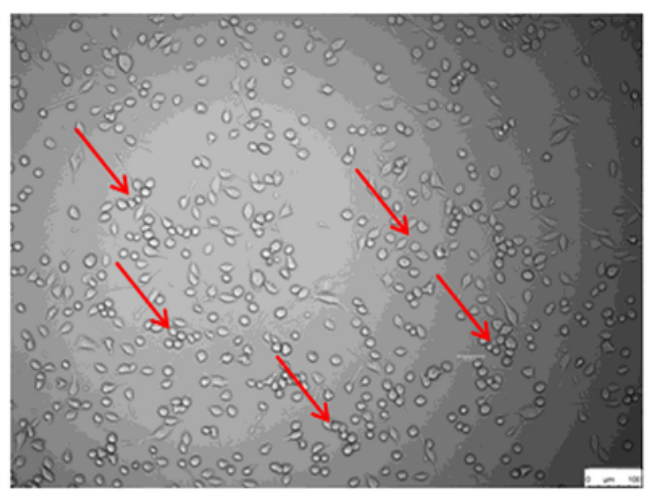

LPS

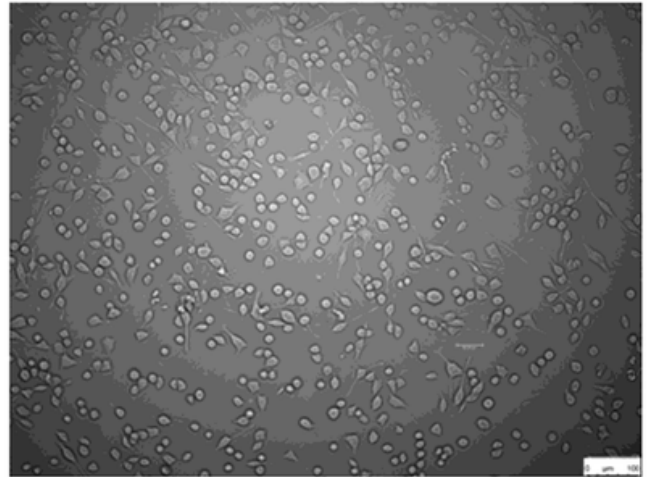

GLGZG $100 \mu \mathrm{g} / \mathrm{mL}$

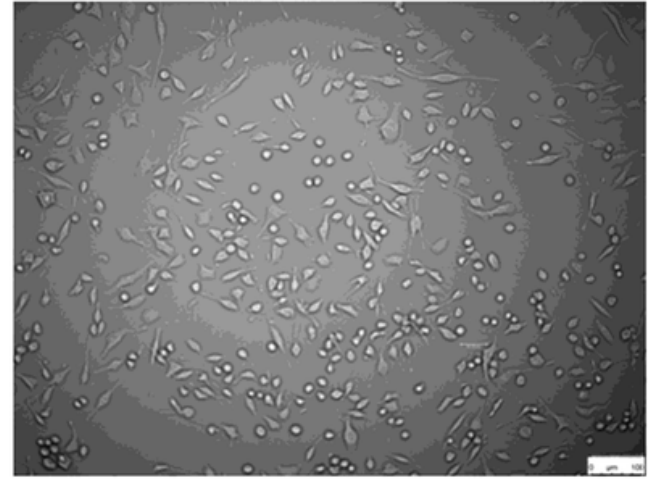

GLGZG $200 \mu \mathrm{g} / \mathrm{mL}$

Figure 2

Effects of Gualou Guizhi Granule (GLGZG) on the morphological characteristics with or without LPS stimulation. Representative bright field microscopy images show the morphological differences among nonactivated control, LPS-activated and activated + GLGZG (50, 100 and $200 \mu \mathrm{g} / \mathrm{mL})$-treated BV2 microglia cells. 


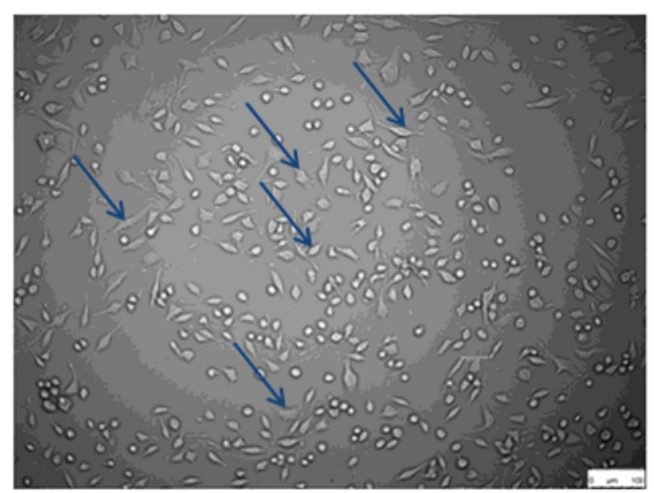

control

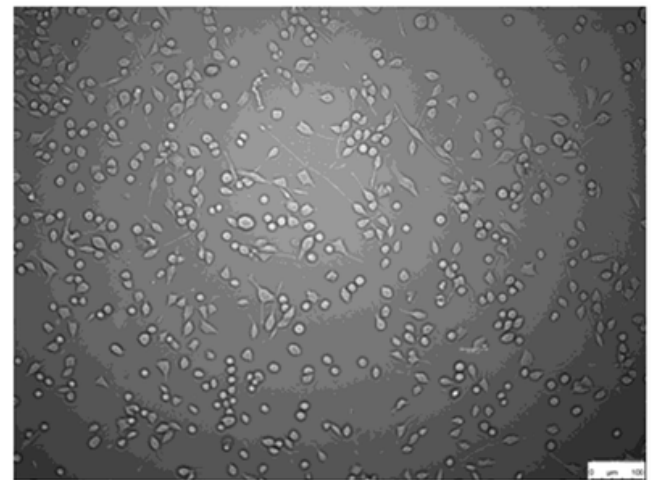

GLGZG $50 \mu \mathrm{g} / \mathrm{mL}$

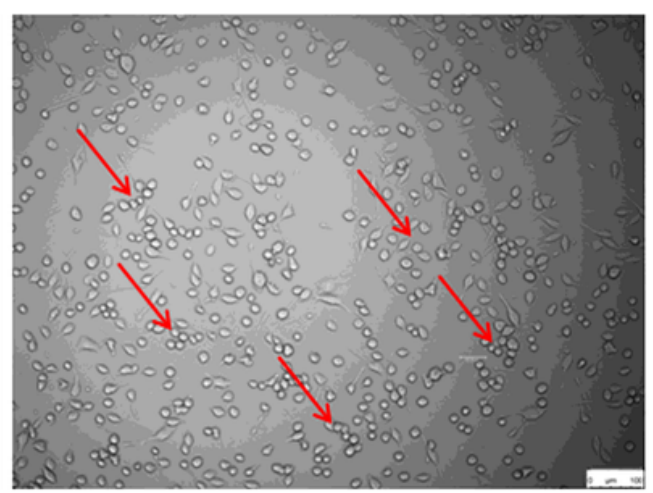

LPS

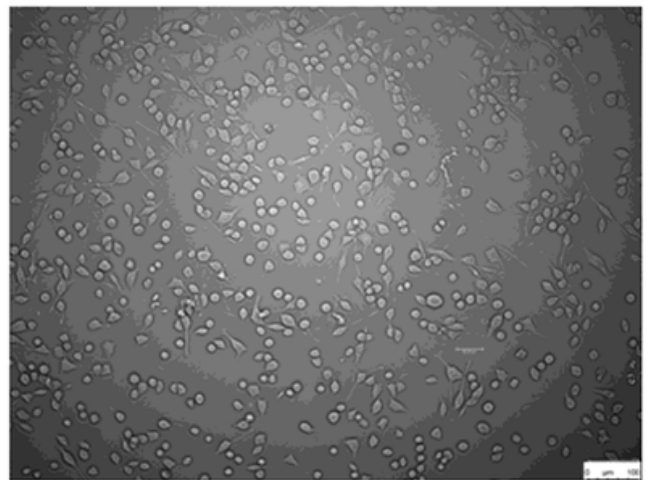

GLGZG $100 \mu \mathrm{g} / \mathrm{mL}$

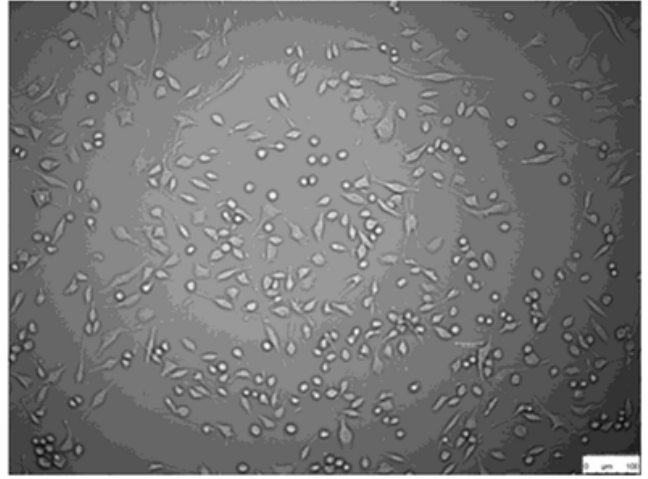

GLGZG $200 \mu \mathrm{g} / \mathrm{mL}$

Figure 2

Effects of Gualou Guizhi Granule (GLGZG) on the morphological characteristics with or without LPS stimulation. Representative bright field microscopy images show the morphological differences among nonactivated control, LPS-activated and activated + GLGZG (50, 100 and $200 \mu \mathrm{g} / \mathrm{mL})$-treated BV2 microglia cells.
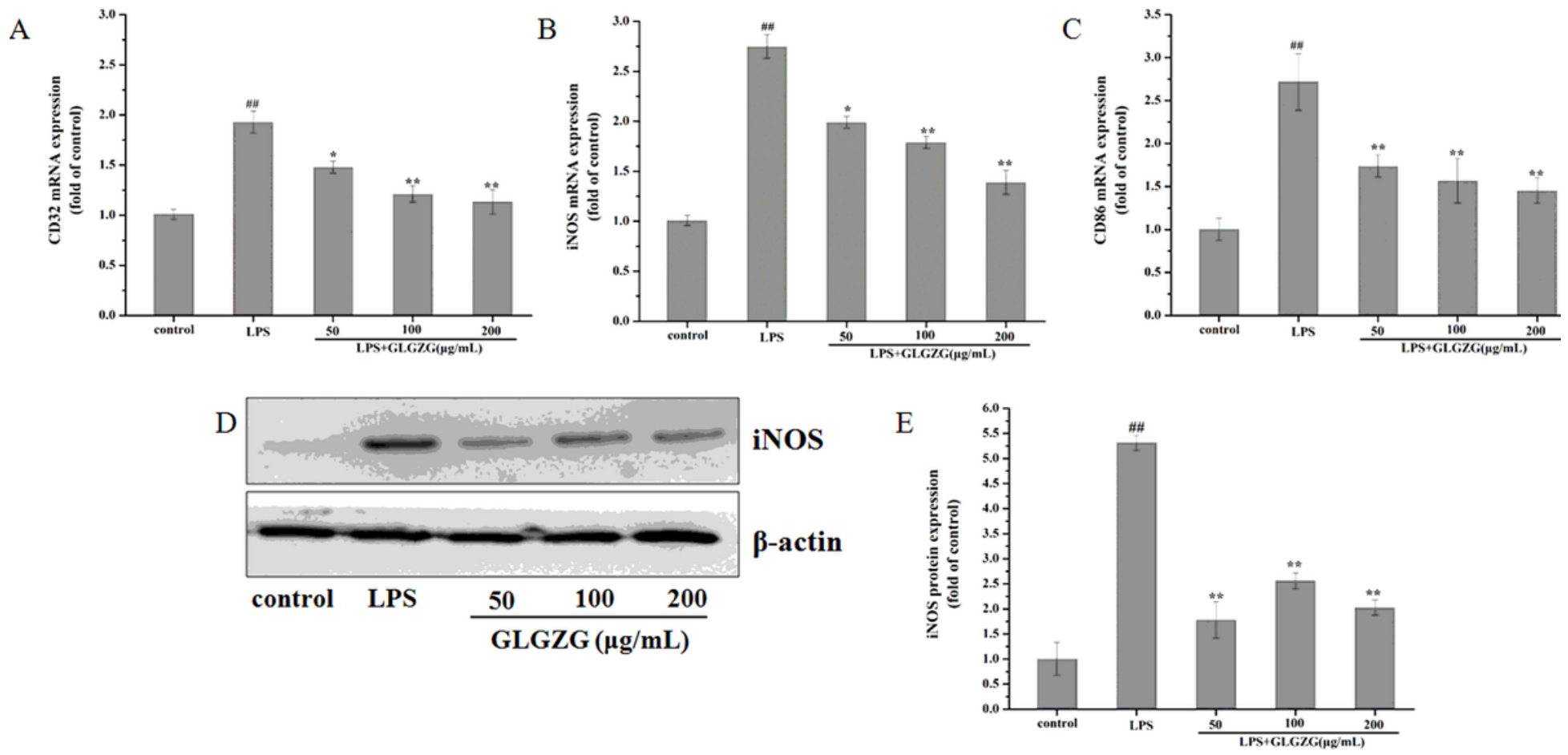


\section{Figure 3}

Suppression of M1 phenotype markers by Gualou Guizhi Granule (GLGZG) in the LPS-activated BV2 microglia cells. (A-C) mRNA was collected from control, LPS-activated and LPS activated + GLGZG (50, 100 and $200 \mu \mathrm{g} / \mathrm{mL}$ )-treated BV2 cells at 24 hours after activation and treatment. (D, E) Western blot analysis and quantification of the M1 marker, iNOS. Data were shown as means $\pm S D$. \#\#P<0.01 versus the control group. ${ }^{*} \mathrm{P}<0.05$ and ${ }^{*} \mathrm{P}<0.01$ versus the LPS-treated group.
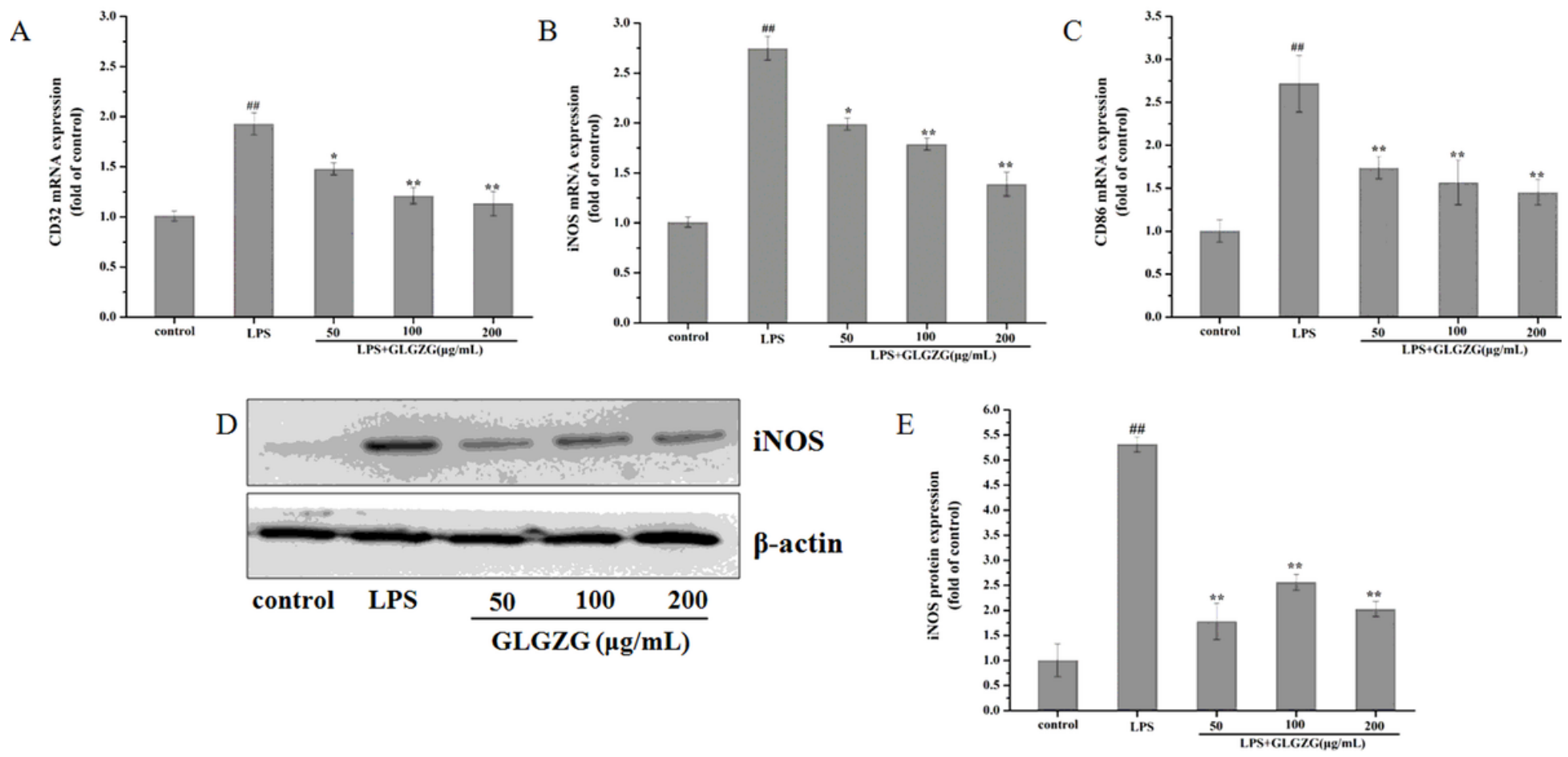

\section{Figure 3}

Suppression of M1 phenotype markers by Gualou Guizhi Granule (GLGZG) in the LPS-activated BV2 microglia cells. (A-C) mRNA was collected from control, LPS-activated and LPS activated + GLGZG (50, 100 and $200 \mu \mathrm{g} / \mathrm{mL}$ )-treated BV2 cells at 24 hours after activation and treatment. (D, E) Western blot analysis and quantification of the M1 marker, iNOS. Data were shown as means $\pm S D$. $\# \# P<0.01$ versus the control group. ${ }^{*} \mathrm{P}<0.05$ and ${ }^{*} \mathrm{P}<0.01$ versus the LPS-treated group. 

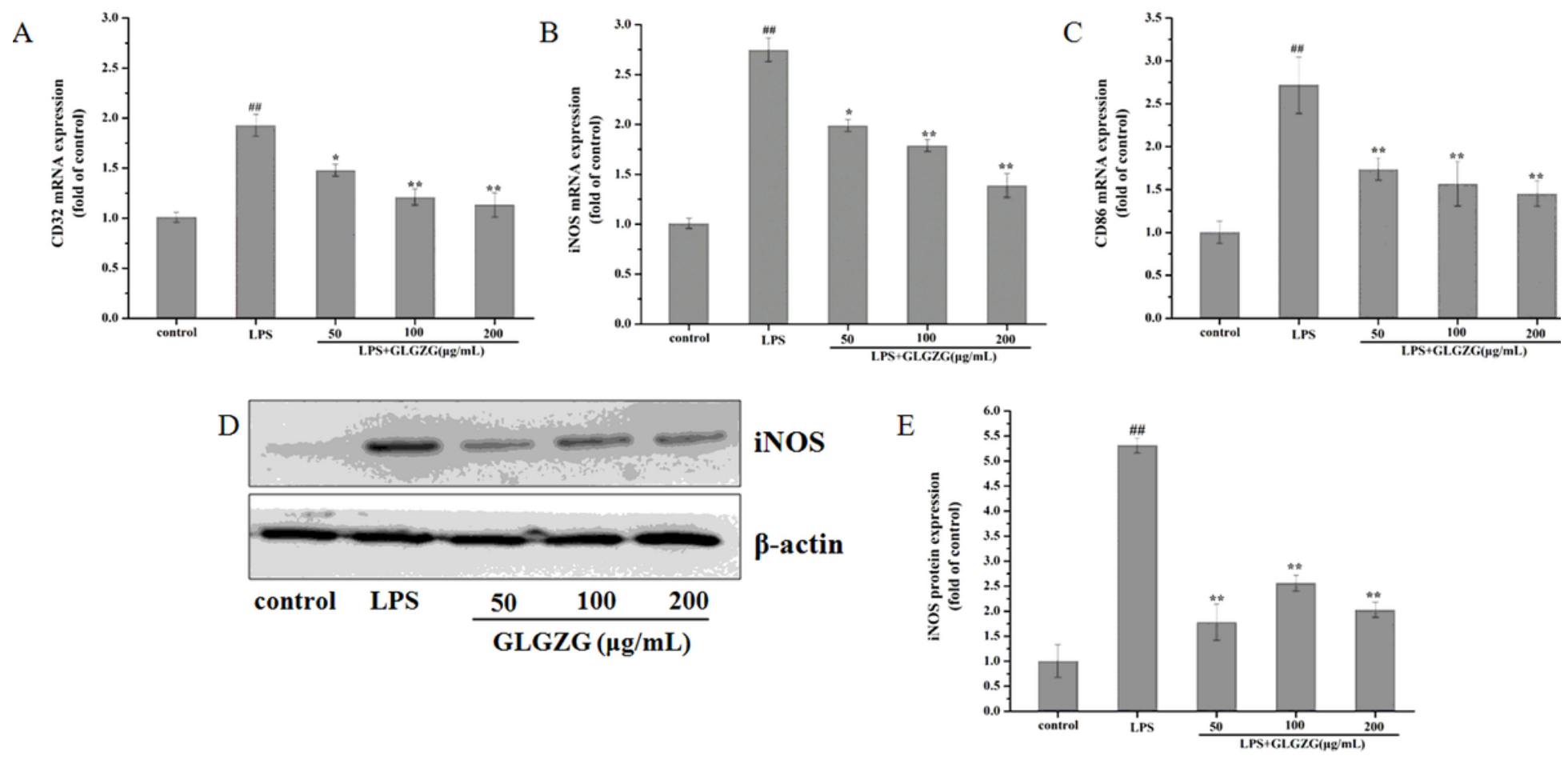

\section{Figure 3}

Suppression of M1 phenotype markers by Gualou Guizhi Granule (GLGZG) in the LPS-activated BV2 microglia cells. (A-C) mRNA was collected from control, LPS-activated and LPS activated + GLGZG (50, 100 and $200 \mu \mathrm{g} / \mathrm{mL}$ )-treated BV2 cells at 24 hours after activation and treatment. (D, E) Western blot analysis and quantification of the M1 marker, iNOS. Data were shown as means $\pm S D$. \#\#P<0.01 versus the control group. ${ }^{*} \mathrm{P}<0.05$ and ${ }^{*} \mathrm{P}<0.01$ versus the LPS-treated group.
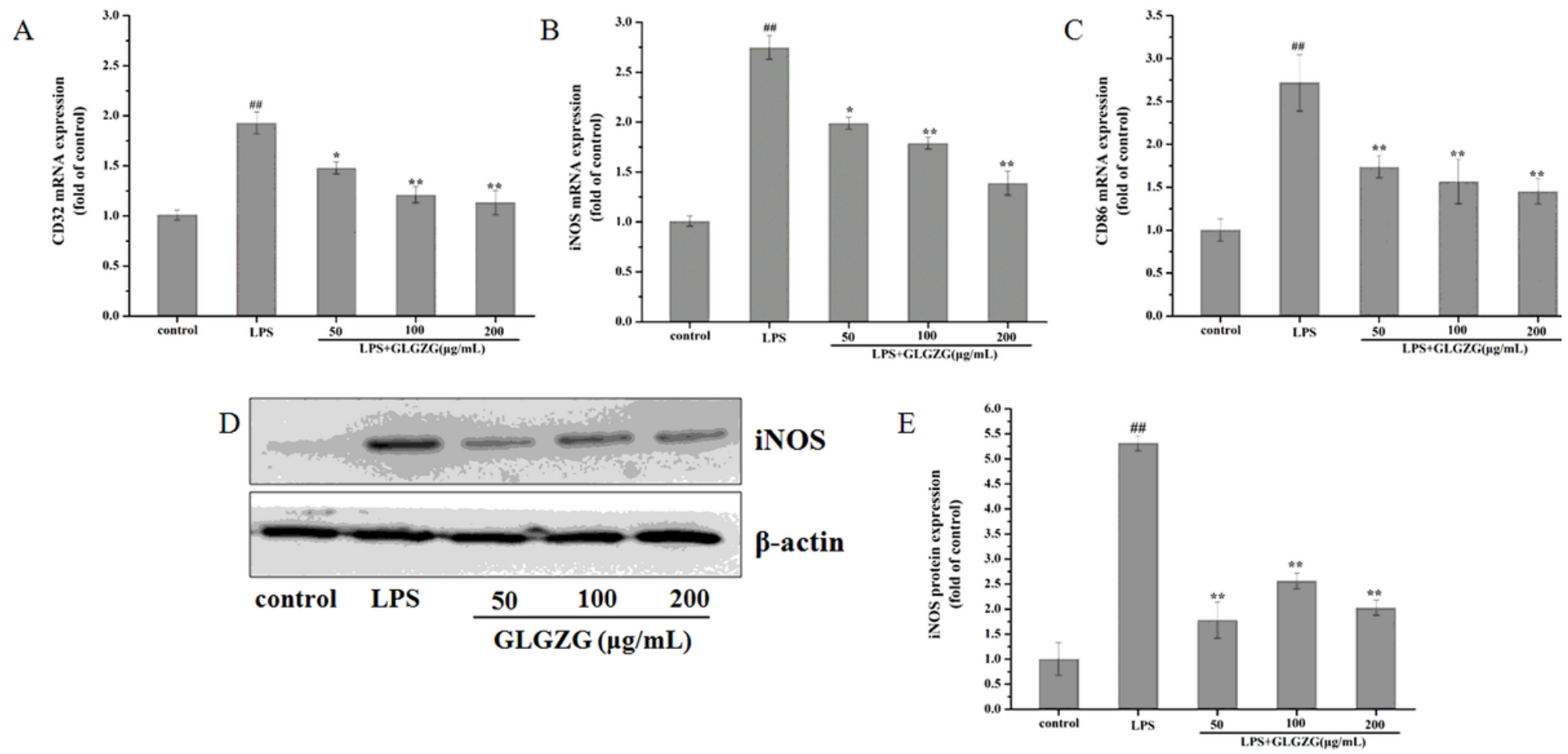

Figure 3 
Suppression of M1 phenotype markers by Gualou Guizhi Granule (GLGZG) in the LPS-activated BV2 microglia cells. (A-C) mRNA was collected from control, LPS-activated and LPS activated + GLGZG (50, 100 and $200 \mu \mathrm{g} / \mathrm{mL}$ )-treated BV2 cells at 24 hours after activation and treatment. (D, E) Western blot analysis and quantification of the M1 marker, iNOS. Data were shown as means $\pm S D$. \#\#P<0.01 versus the control group. ${ }^{*} \mathrm{P}<0.05$ and ${ }^{*} \mathrm{P}<0.01$ versus the LPS-treated group.
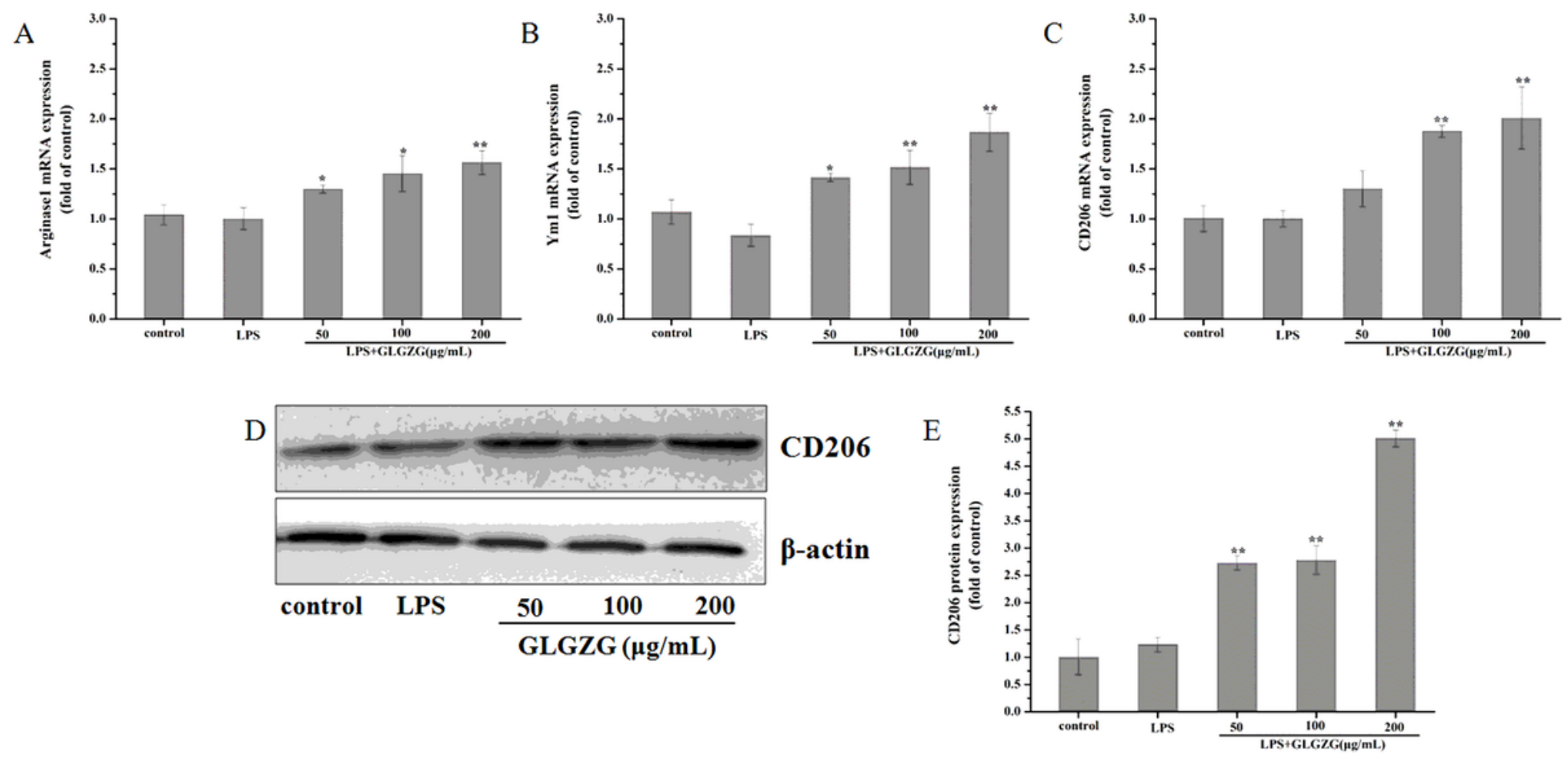

\section{Figure 4}

Upregulation of M2 phenotype markers by Gualou Guizhi Granule (GLGZG) in the LPS-activated BV2 microglia cells. (A-C) mRNA was collected from control, LPS-activated and LPS activated + GLGZG (50, 100 and $200 \mu \mathrm{g} / \mathrm{mL}$ )-treated BV2 cells at 24 hours after activation and treatment. (D, E) Western blot analysis and quantification of the M2 marker, CD206. Data were shown as means $\pm S D$. ${ }^{*}<<0.05$ and $\star \star P<0.01$ versus the LPS-treated group. 

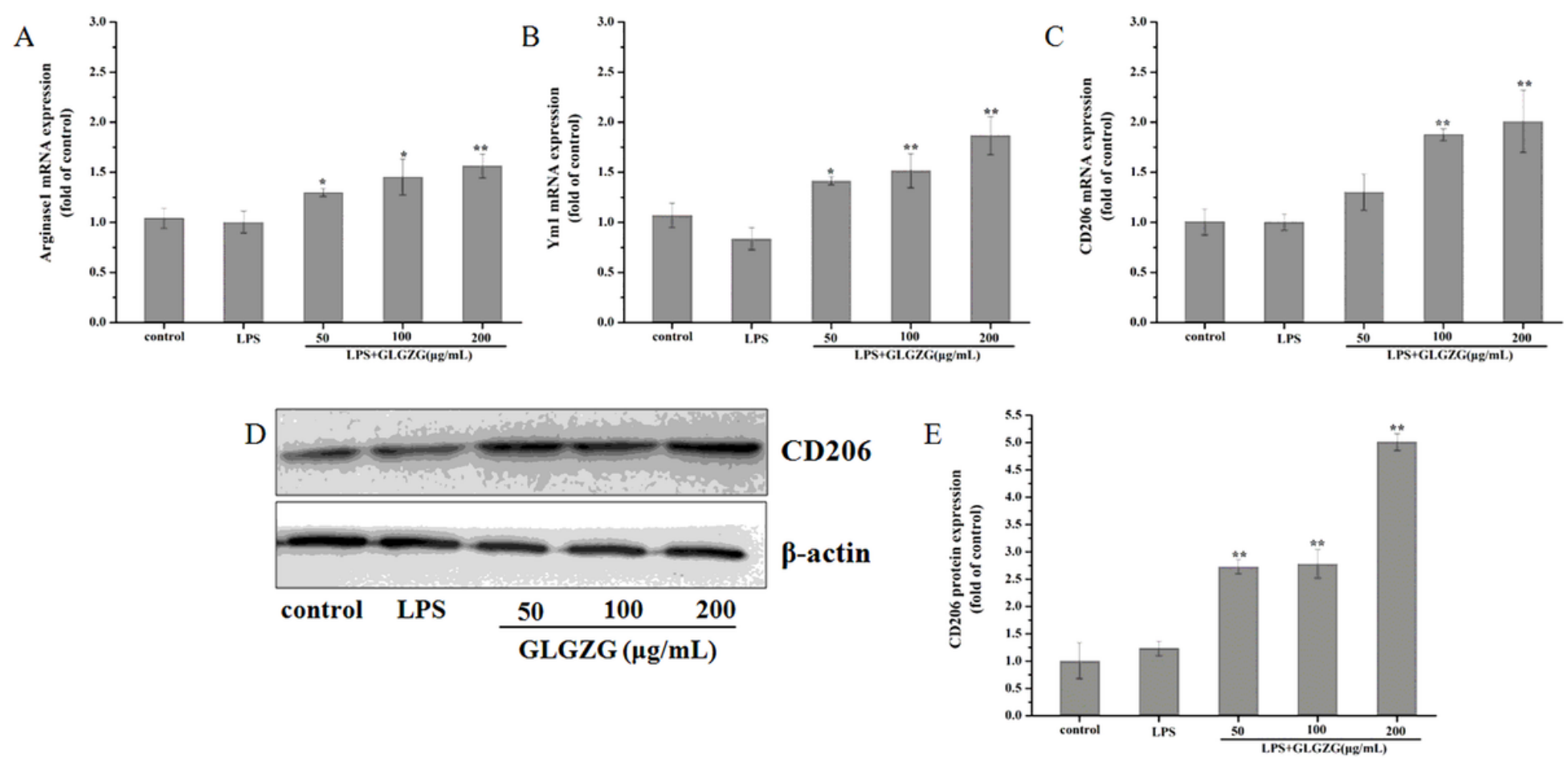

Figure 4

Upregulation of M2 phenotype markers by Gualou Guizhi Granule (GLGZG) in the LPS-activated BV2 microglia cells. (A-C) mRNA was collected from control, LPS-activated and LPS activated + GLGZG (50, 100 and $200 \mu \mathrm{g} / \mathrm{mL}$ )-treated BV2 cells at 24 hours after activation and treatment. (D, E) Western blot analysis and quantification of the M2 marker, CD206. Data were shown as means $\pm S D$. ${ }^{*} P<0.05$ and $* * P<0.01$ versus the LPS-treated group.
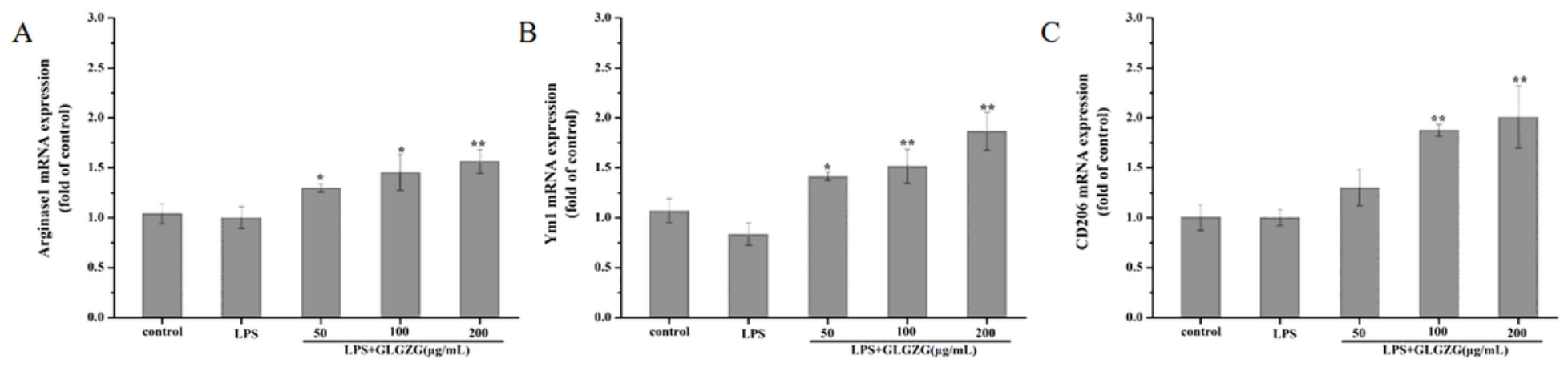

$\mathrm{D}$

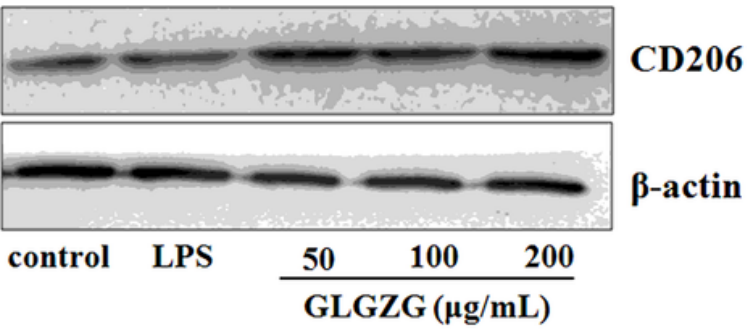

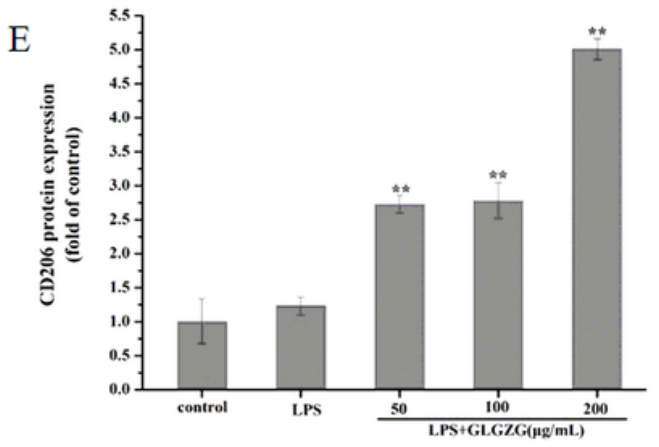

Figure 4 
Upregulation of M2 phenotype markers by Gualou Guizhi Granule (GLGZG) in the LPS-activated BV2 microglia cells. (A-C) mRNA was collected from control, LPS-activated and LPS activated + GLGZG (50, 100 and $200 \mu \mathrm{g} / \mathrm{mL}$ )-treated BV2 cells at 24 hours after activation and treatment. (D, E) Western blot analysis and quantification of the M2 marker, CD206. Data were shown as means $\pm S D$. ${ }^{*} P<0.05$ and $* * P<0.01$ versus the LPS-treated group.
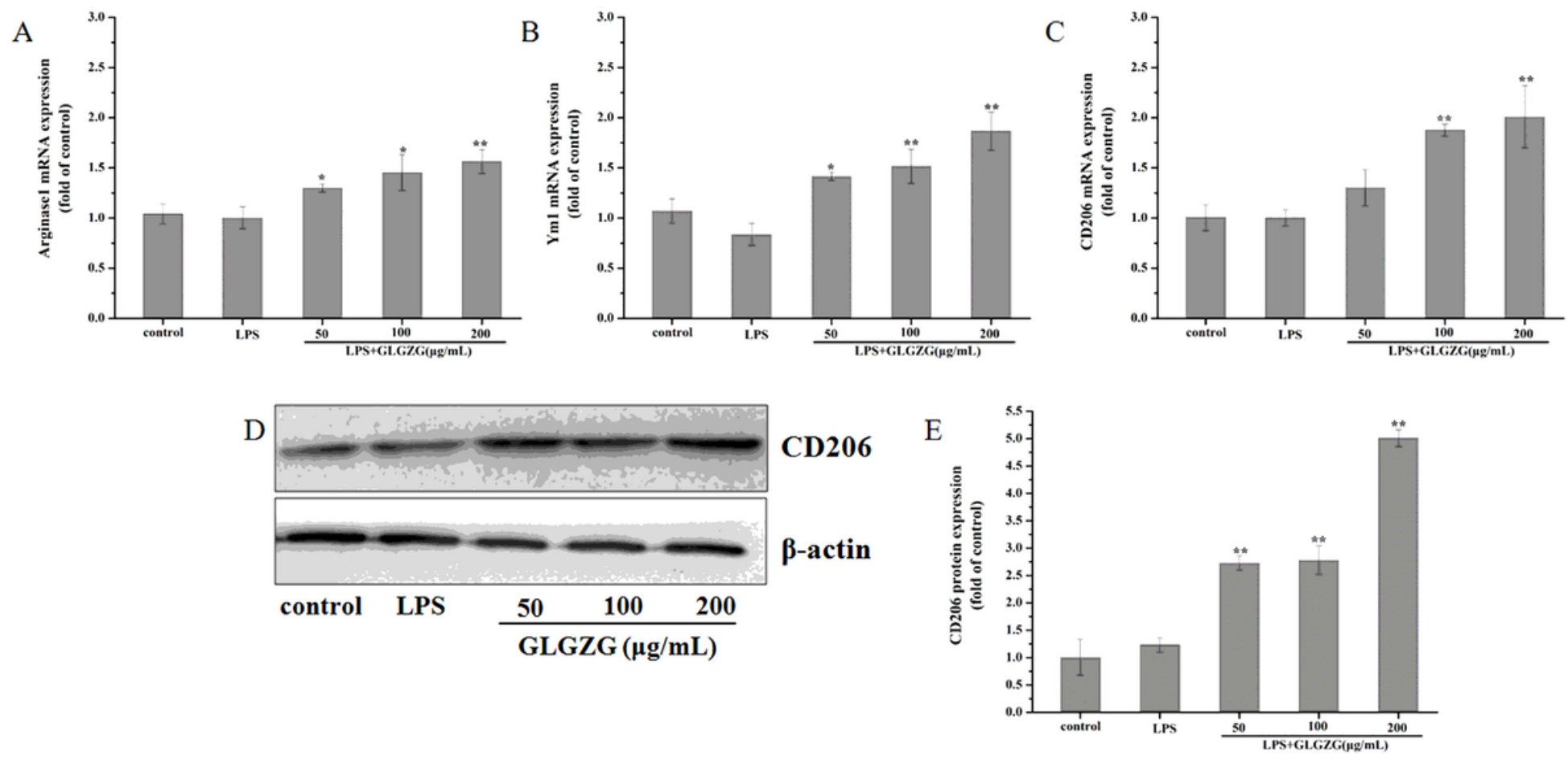

\section{Figure 4}

Upregulation of M2 phenotype markers by Gualou Guizhi Granule (GLGZG) in the LPS-activated BV2 microglia cells. (A-C) mRNA was collected from control, LPS-activated and LPS activated + GLGZG (50, 100 and $200 \mu \mathrm{g} / \mathrm{mL}$ )-treated BV2 cells at 24 hours after activation and treatment. (D, E) Western blot analysis and quantification of the M2 marker, CD206. Data were shown as means $\pm S D$. ${ }^{*} P<0.05$ and $\star \star P<0.01$ versus the LPS-treated group. 
A

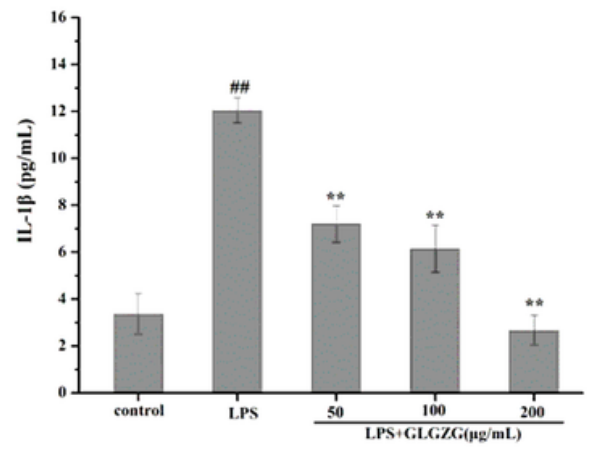

$\mathrm{D}$

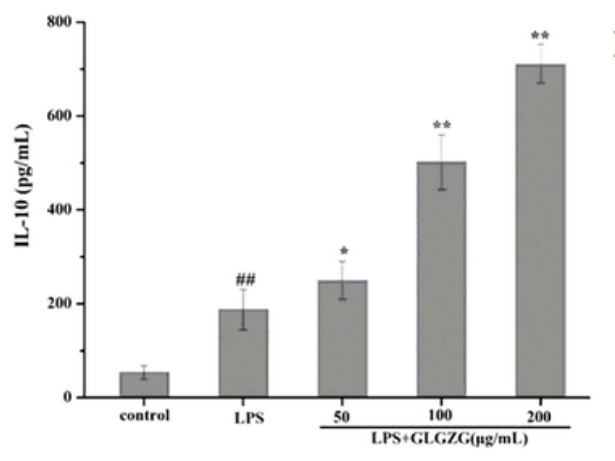

B

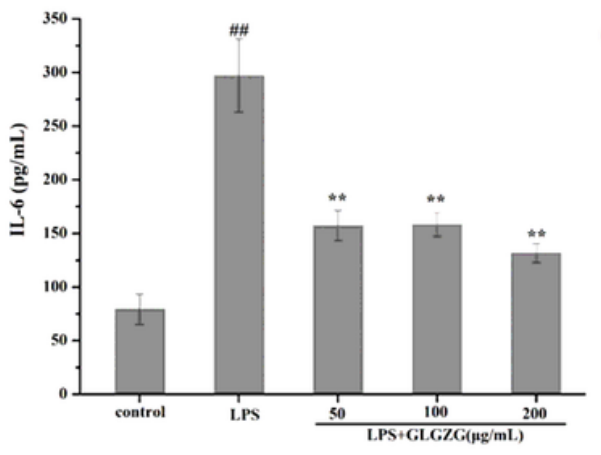

$\mathrm{C}$

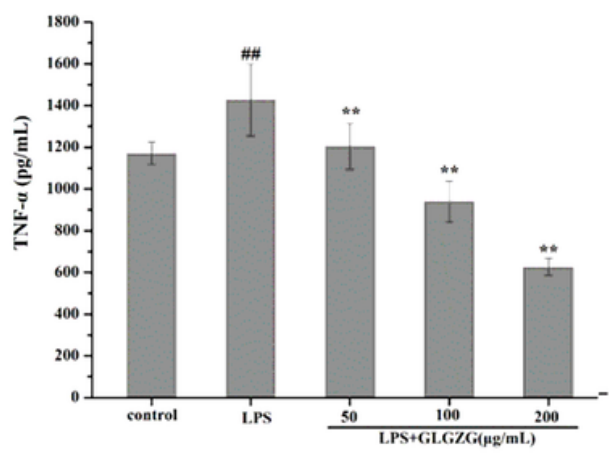

Figure 5
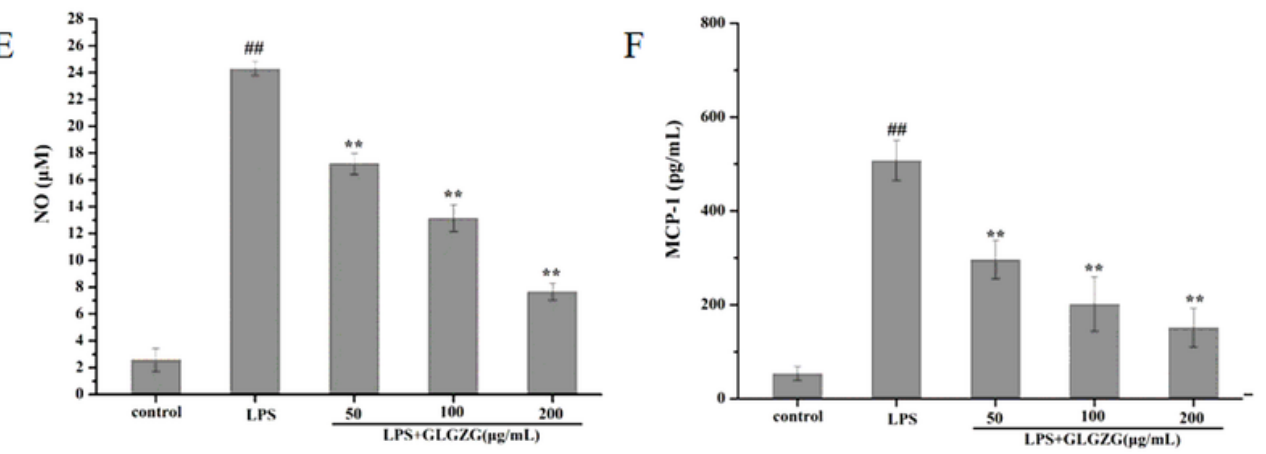

Effect of Gualou Guizhi Granule (GLGZG) on the secretion of IL-1 $\beta$, IL-6, IL-10, TNF-a, NO and MCP-1 in LPS-stimulated BV2. Cells were treated with 50,100 and $200 \mu \mathrm{g} / \mathrm{mL}$ of GLGZG and incubated in the presence of $1 \mu \mathrm{g} / \mathrm{mL}$ LPS for $24 \mathrm{~h}$. Data were shown as means \pm SD. \#\#P<0.01 versus the control group. ${ }^{*} \mathrm{P}<0.05$ and ${ }^{* *} \mathrm{P}<0.01$ versus the LPS-treated group.

A

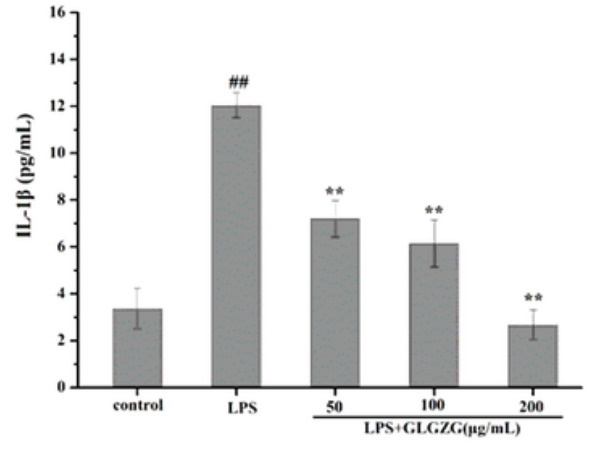

$\mathrm{D}$

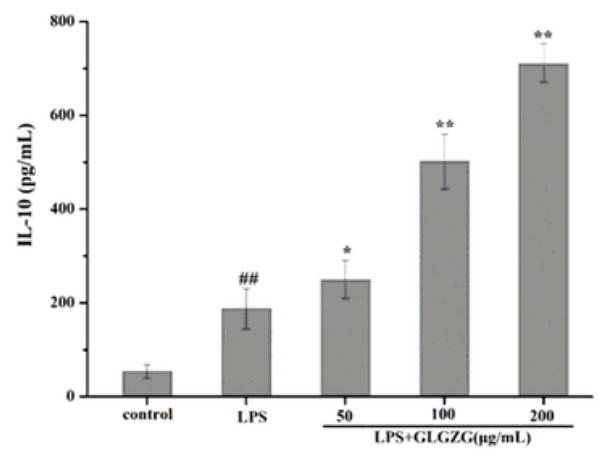

B
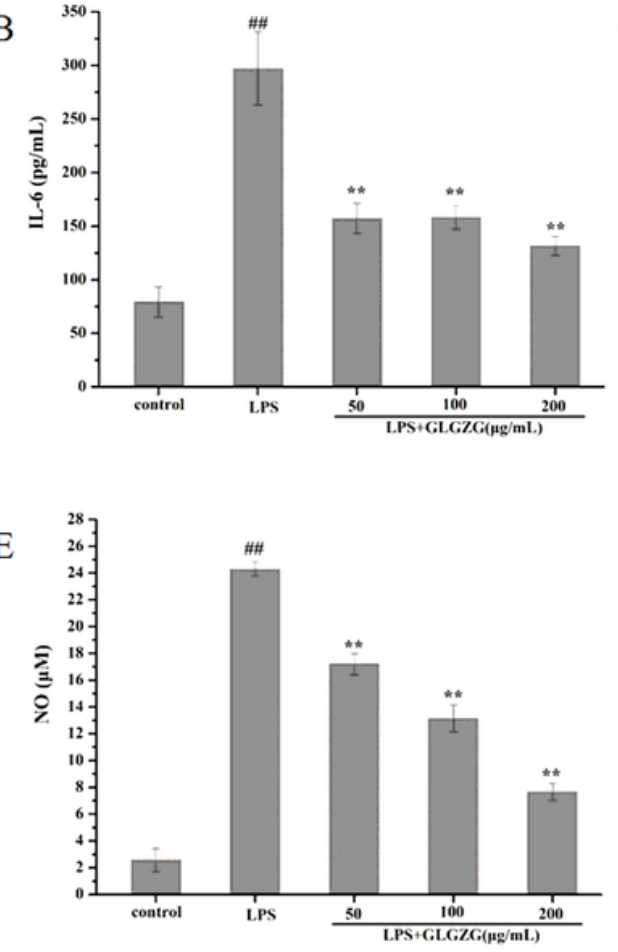

$\mathrm{C}$

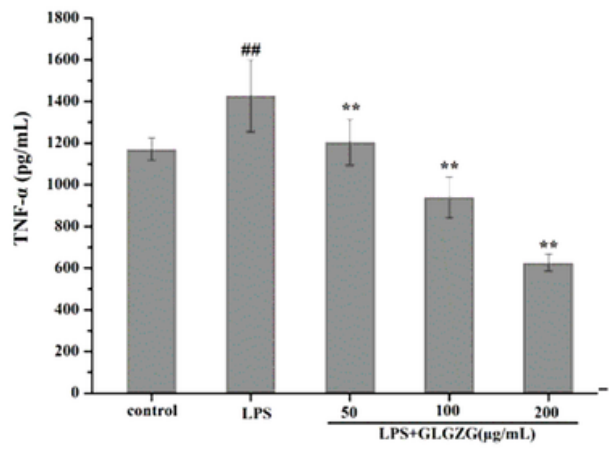

$\mathrm{F}$

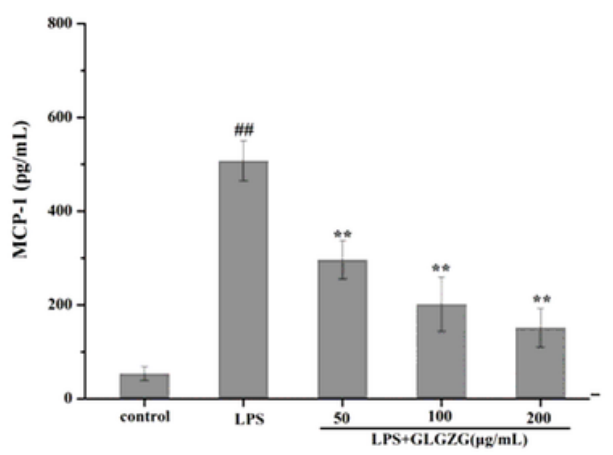

Figure 5 
Effect of Gualou Guizhi Granule (GLGZG) on the secretion of IL-1 $\beta$, IL-6, IL-10, TNF- $\alpha$, NO and MCP-1 in LPS-stimulated BV2. Cells were treated with 50,100 and $200 \mu \mathrm{g} / \mathrm{mL}$ of GLGZG and incubated in the presence of $1 \mu \mathrm{g} / \mathrm{mL}$ LPS for $24 \mathrm{~h}$. Data were shown as means \pm SD. \#\#P<0.01 versus the control group. ${ }^{*} \mathrm{P}<0.05$ and ${ }^{*} \mathrm{P}<0.01$ versus the LPS-treated group.

A

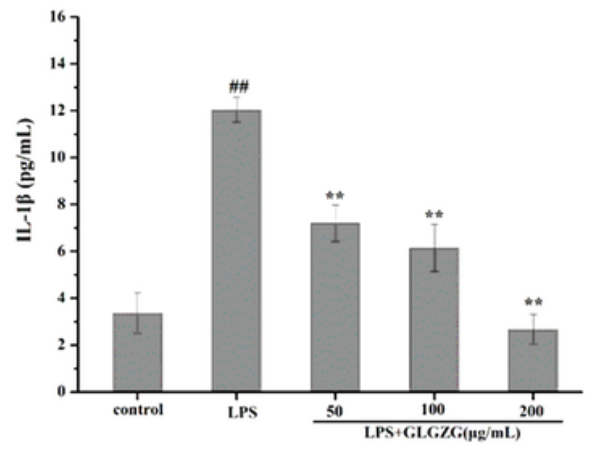

$\mathrm{D}$

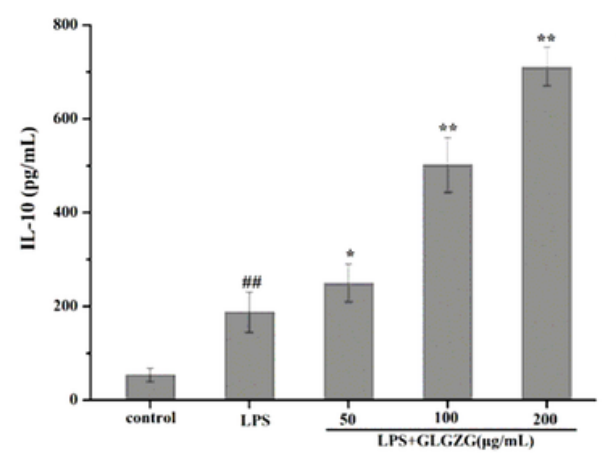

B

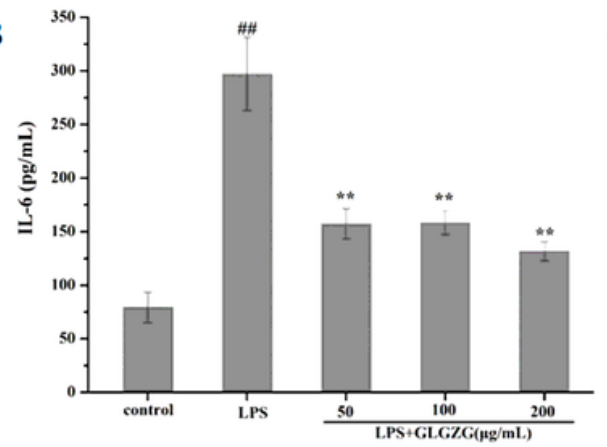

E

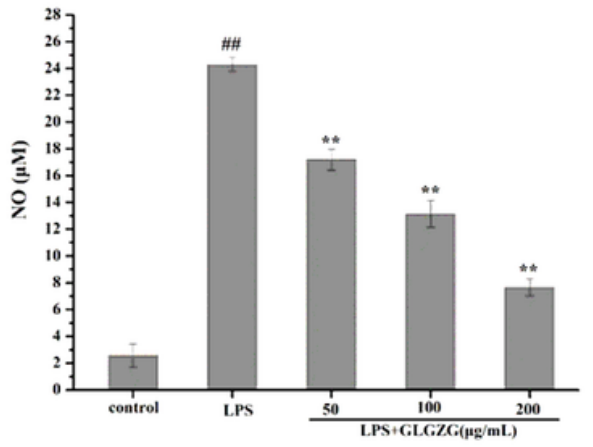

$\mathrm{C}$

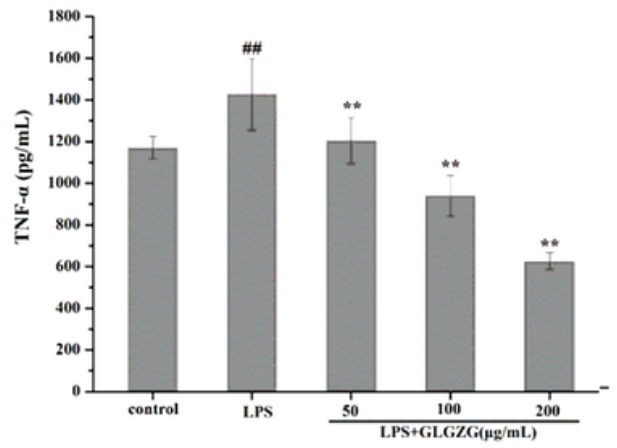

$\mathrm{F}$

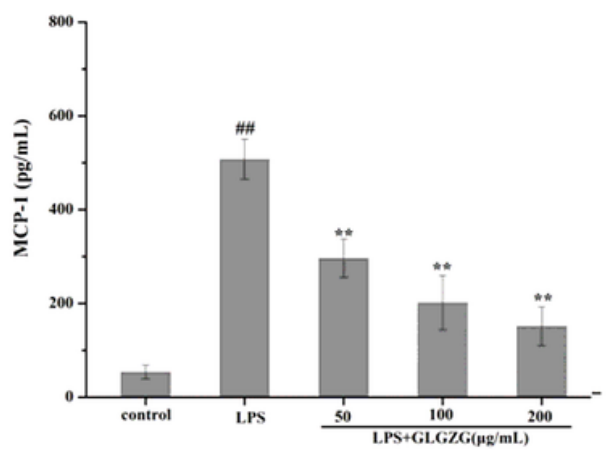

Figure 5

Effect of Gualou Guizhi Granule (GLGZG) on the secretion of IL-1 $\beta$, IL-6, IL-10, TNF- $\alpha$, NO and MCP-1 in LPS-stimulated BV2. Cells were treated with 50,100 and $200 \mu \mathrm{g} / \mathrm{mL}$ of GLGZG and incubated in the presence of $1 \mu \mathrm{g} / \mathrm{mL}$ LPS for $24 \mathrm{~h}$. Data were shown as means $\pm S D$. \#\#P<0.01 versus the control group. ${ }^{*} \mathrm{P}<0.05$ and ${ }^{* \star} \mathrm{P}<0.01$ versus the LPS-treated group. 
A

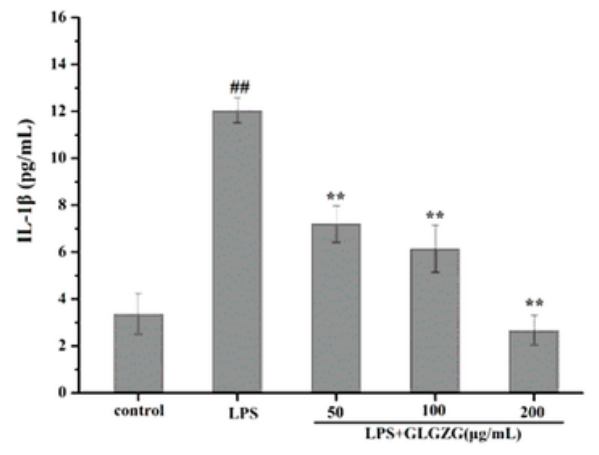

$\mathrm{D}$

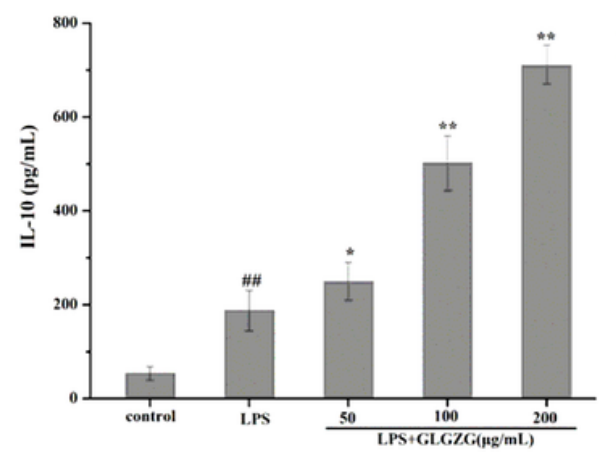

B

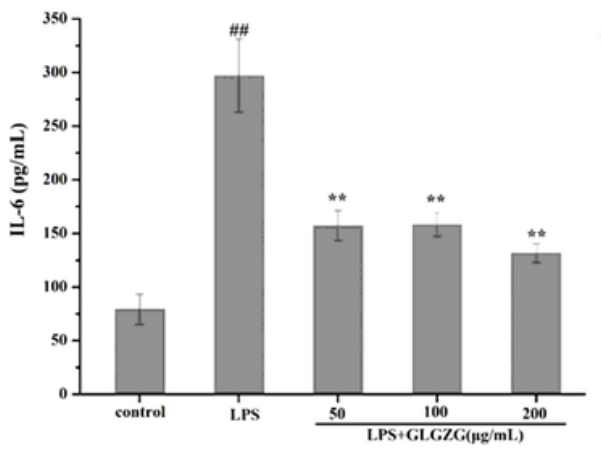

$\mathrm{C}$

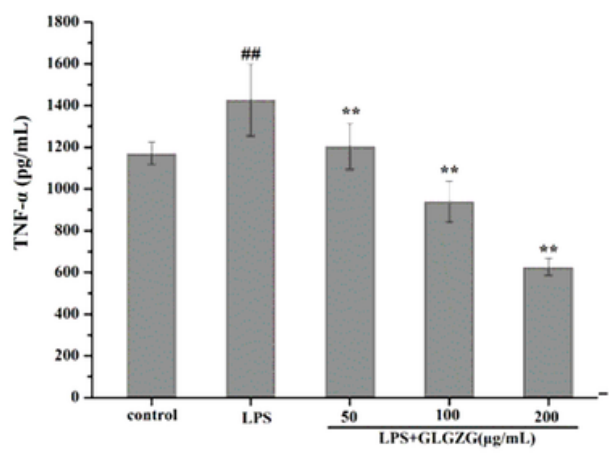

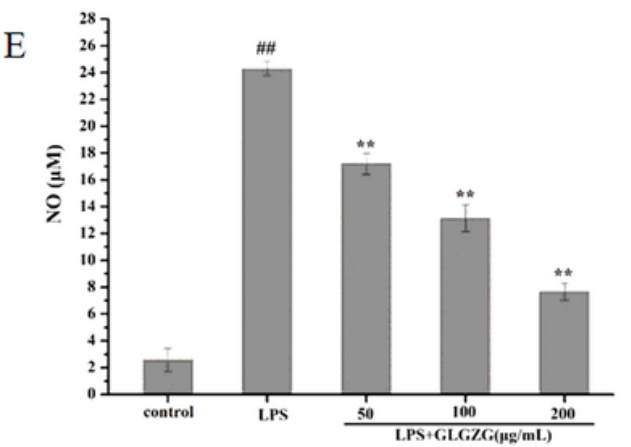

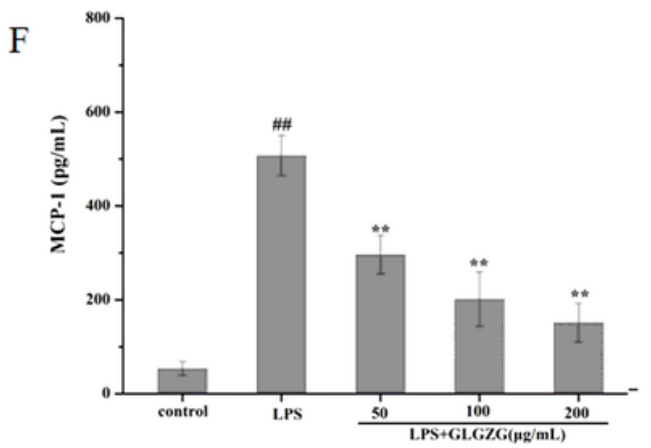

Figure 5

Effect of Gualou Guizhi Granule (GLGZG) on the secretion of IL-1 $\beta$, IL-6, IL-10, TNF-a, NO and MCP-1 in LPS-stimulated BV2. Cells were treated with 50,100 and $200 \mu \mathrm{g} / \mathrm{mL}$ of GLGZG and incubated in the presence of $1 \mu \mathrm{g} / \mathrm{mL}$ LPS for $24 \mathrm{~h}$. Data were shown as means \pm SD. \#\#P<0.01 versus the control group. ${ }^{*} P<0.05$ and ${ }^{* *} P<0.01$ versus the LPS-treated group.
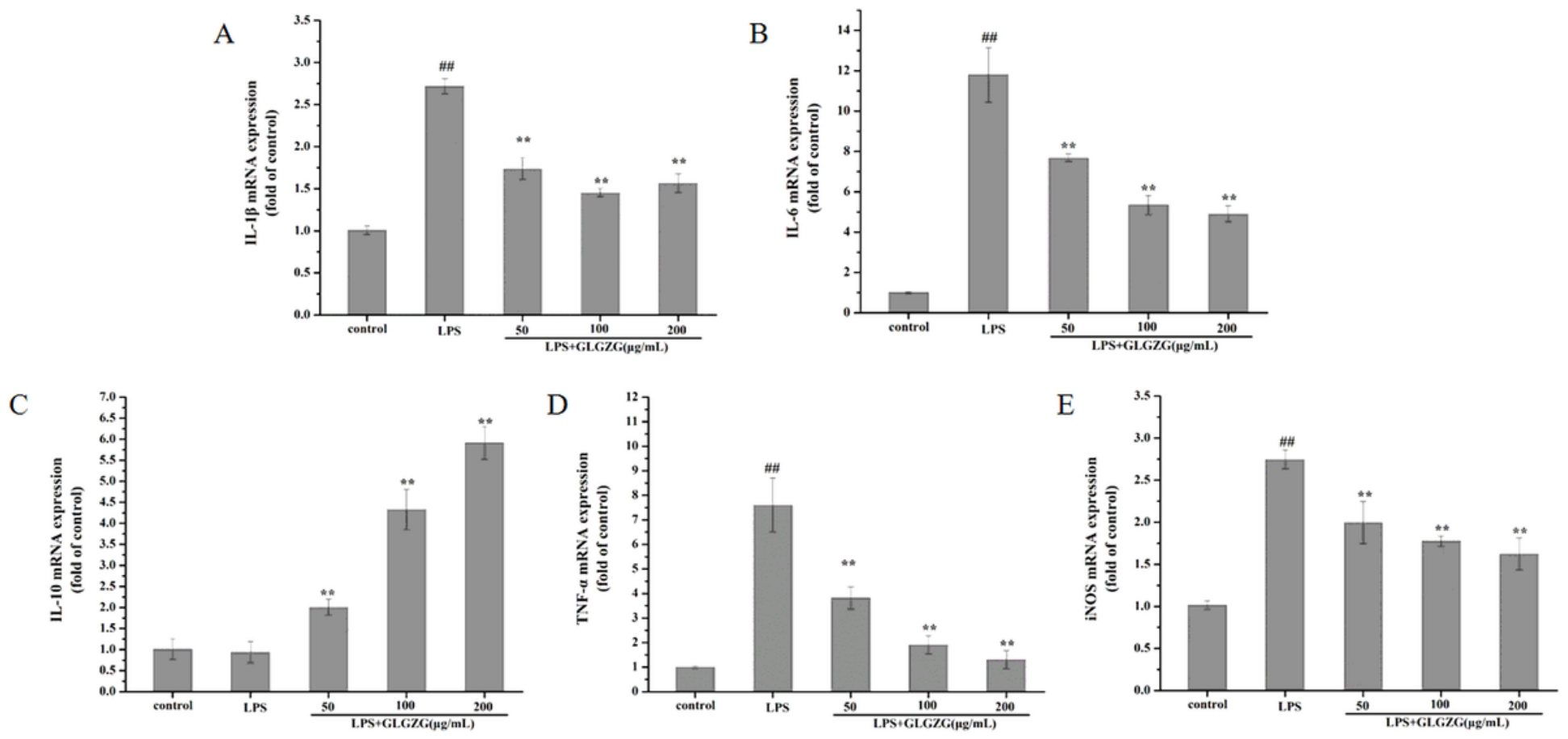

Figure 6 
Effect of Gualou Guizhi Granule (GLGZG) on mRNA expression of IL-1 $\beta$, IL-6, IL-10, TNF- $\alpha$ and iNOS in LPS-stimulated BV2. Cells were treated with 50,100 and $200 \mu \mathrm{g} / \mathrm{mL}$ of GLGZG and incubated in the presence of $1 \mu \mathrm{g} / \mathrm{mL}$ LPS for $24 \mathrm{~h}$. The mRNA levels were expressed as relative fold to the control group. Data were shown as means \pm SD. ${ }^{*} \mathrm{P}<0.01$ versus the LPS-treated group.
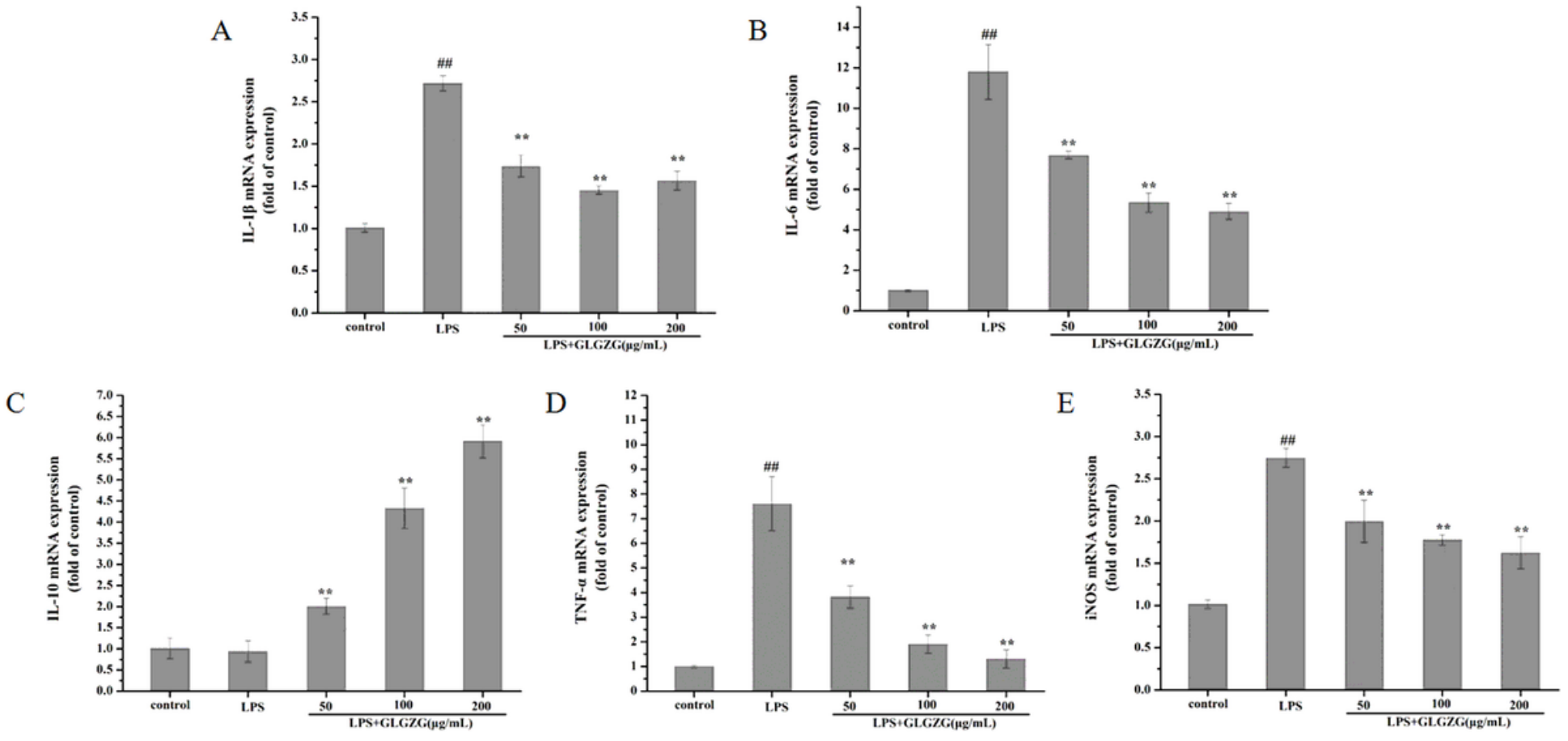

Figure 6

Effect of Gualou Guizhi Granule (GLGZG) on mRNA expression of IL-1 $\beta$, IL-6, IL-10, TNF- $\alpha$ and iNOS in LPS-stimulated BV2. Cells were treated with 50,100 and $200 \mu \mathrm{g} / \mathrm{mL}$ of GLGZG and incubated in the presence of $1 \mu \mathrm{g} / \mathrm{mL}$ LPS for $24 \mathrm{~h}$. The mRNA levels were expressed as relative fold to the control group. Data were shown as means \pm SD. $* * P<0.01$ versus the LPS-treated group.
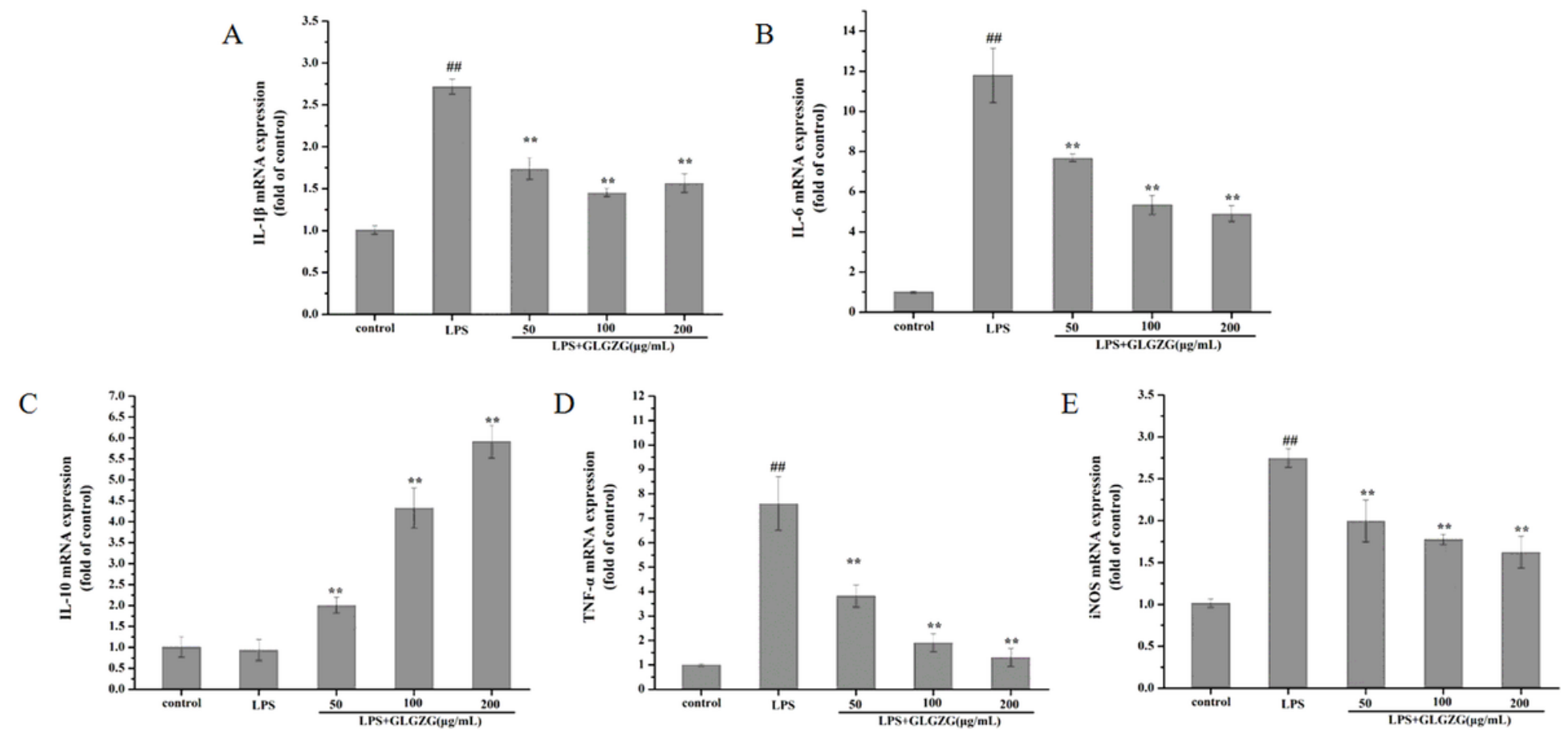
Figure 6

Effect of Gualou Guizhi Granule (GLGZG) on mRNA expression of IL-1 $\beta$, IL-6, IL-10, TNF- $\alpha$ and iNOS in LPS-stimulated BV2. Cells were treated with 50,100 and $200 \mu \mathrm{g} / \mathrm{mL}$ of GLGZG and incubated in the presence of $1 \mu \mathrm{g} / \mathrm{mL}$ LPS for $24 \mathrm{~h}$. The mRNA levels were expressed as relative fold to the control group. Data were shown as means \pm SD. $* * P<0.01$ versus the LPS-treated group.
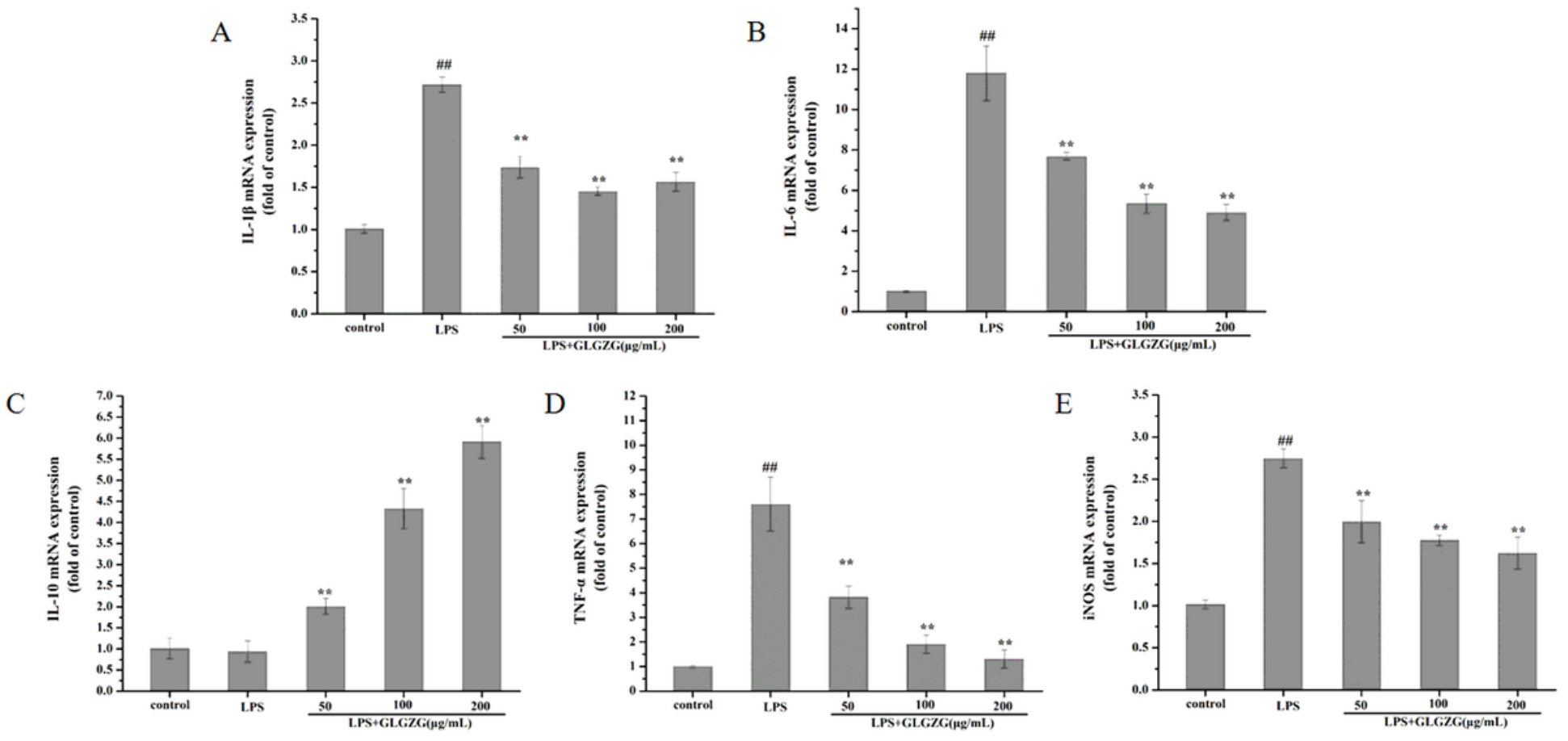

\section{Figure 6}

Effect of Gualou Guizhi Granule (GLGZG) on mRNA expression of IL-1 $\beta$, IL-6, IL-10, TNF- $\alpha$ and iNOS in LPS-stimulated BV2. Cells were treated with 50,100 and $200 \mu \mathrm{g} / \mathrm{mL}$ of GLGZG and incubated in the presence of $1 \mu \mathrm{g} / \mathrm{mL}$ LPS for $24 \mathrm{~h}$. The mRNA levels were expressed as relative fold to the control group. Data were shown as means \pm SD. $* * P<0.01$ versus the LPS-treated group. 

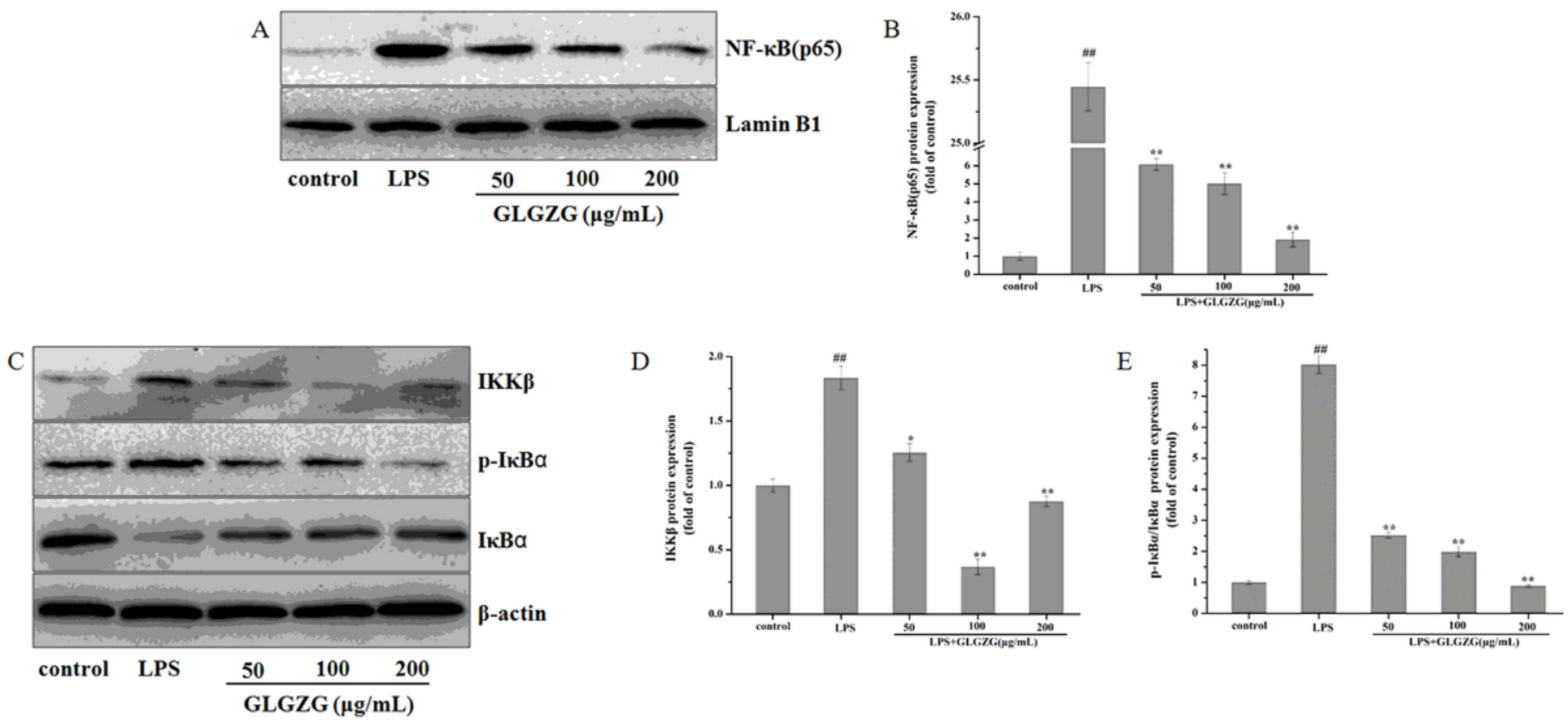

Figure 7

Effect of Gualou Guizhi Granule (GLGZG) on NF-KB signaling in LPS-stimulated BV2. Cells were treated with 50,100 and $200 \mu \mathrm{g} / \mathrm{mL}$ of GLGZG and incubated in the presence of $1 \mu \mathrm{g} / \mathrm{mL}$ LPS for $24 \mathrm{~h}$. After incubation, the protein was extracted from cells, and the levels of NF-KBp65 (A, B) in the nucleus, IkK $(C$, $D), p-I K B$ and $I K B(C, E)$ were measured by Western blot. Data were shown as means $\pm S D$. \#\#P<0.01 versus the control group. ${ }^{*} \mathrm{P}<0.05$ and ${ }^{*}{ }^{*} \mathrm{P}<0.01$ versus the LPS-treated group.
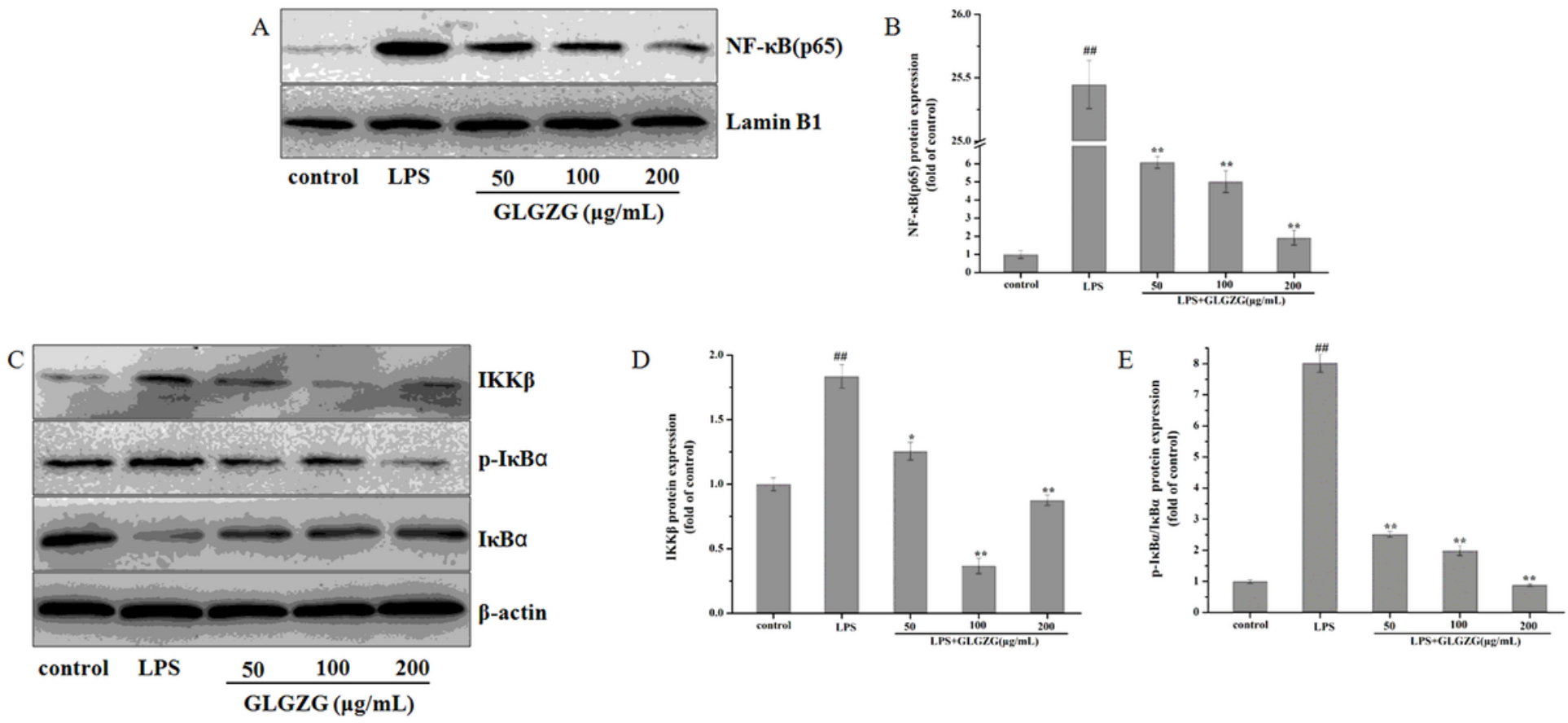

Figure 7 
Effect of Gualou Guizhi Granule (GLGZG) on NF-KB signaling in LPS-stimulated BV2. Cells were treated with 50,100 and $200 \mu \mathrm{g} / \mathrm{mL}$ of GLGZG and incubated in the presence of $1 \mu \mathrm{g} / \mathrm{mL}$ LPS for $24 \mathrm{~h}$. After incubation, the protein was extracted from cells, and the levels of NF-KBp65 (A, B) in the nucleus, IkK $(C$, $D), p-I_{\kappa} B$ and $I K B(C, E)$ were measured by Western blot. Data were shown as means $\pm S D$. \#\#P<0.01 versus the control group. ${ }^{*} \mathrm{P}<0.05$ and ${ }^{*} \mathrm{P}<0.01$ versus the LPS-treated group.
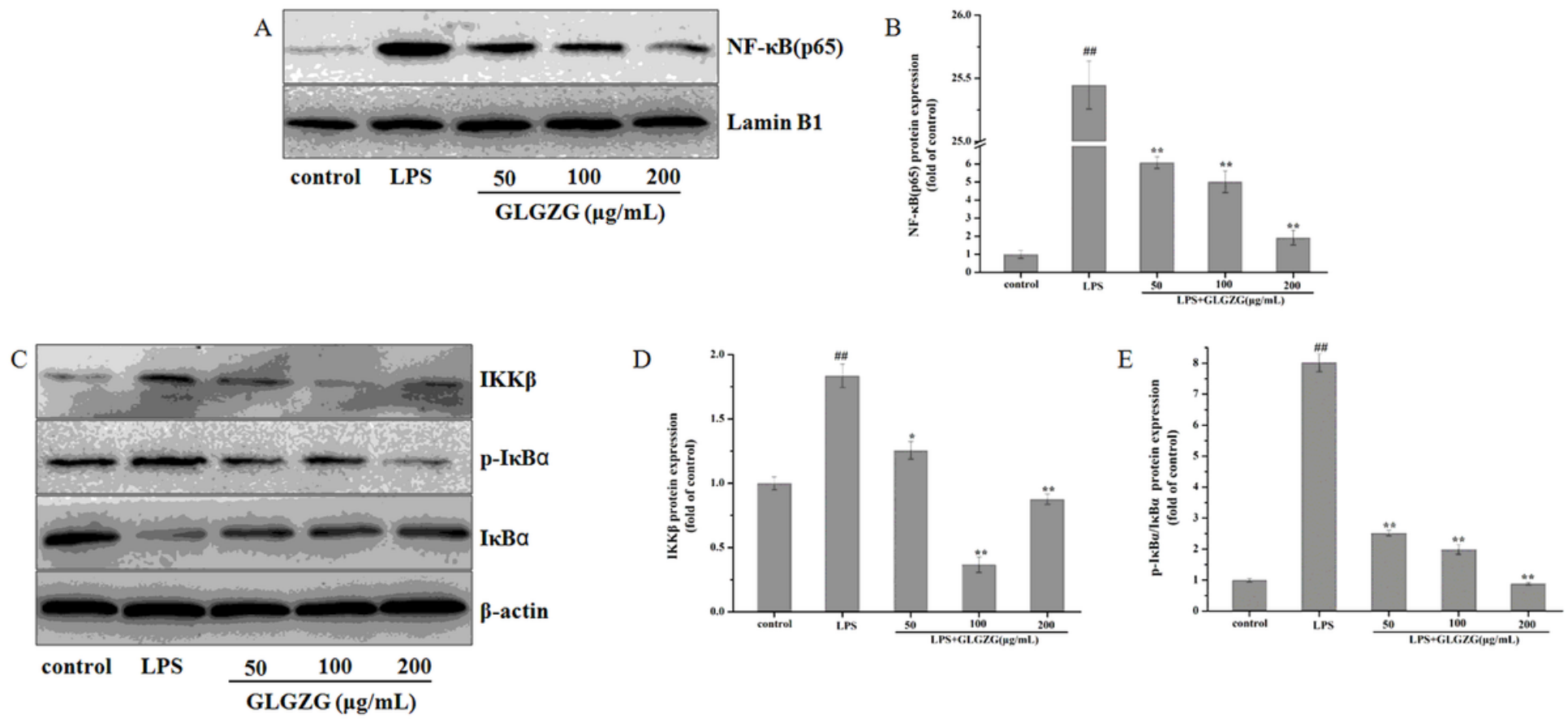

\section{Figure 7}

Effect of Gualou Guizhi Granule (GLGZG) on NF-KB signaling in LPS-stimulated BV2. Cells were treated with 50,100 and $200 \mu \mathrm{g} / \mathrm{mL}$ of GLGZG and incubated in the presence of $1 \mu \mathrm{g} / \mathrm{mL}$ LPS for $24 \mathrm{~h}$. After incubation, the protein was extracted from cells, and the levels of NF-KBp65 (A, B) in the nucleus, IkK $(C$, $D), p-I_{k B}$ and $\operatorname{IKB}(C, E)$ were measured by Western blot. Data were shown as means $\pm S D$. \#\#P<0.01 versus the control group. ${ }^{*} \mathrm{P}<0.05$ and ${ }^{*} * \mathrm{P}<0.01$ versus the LPS-treated group. 

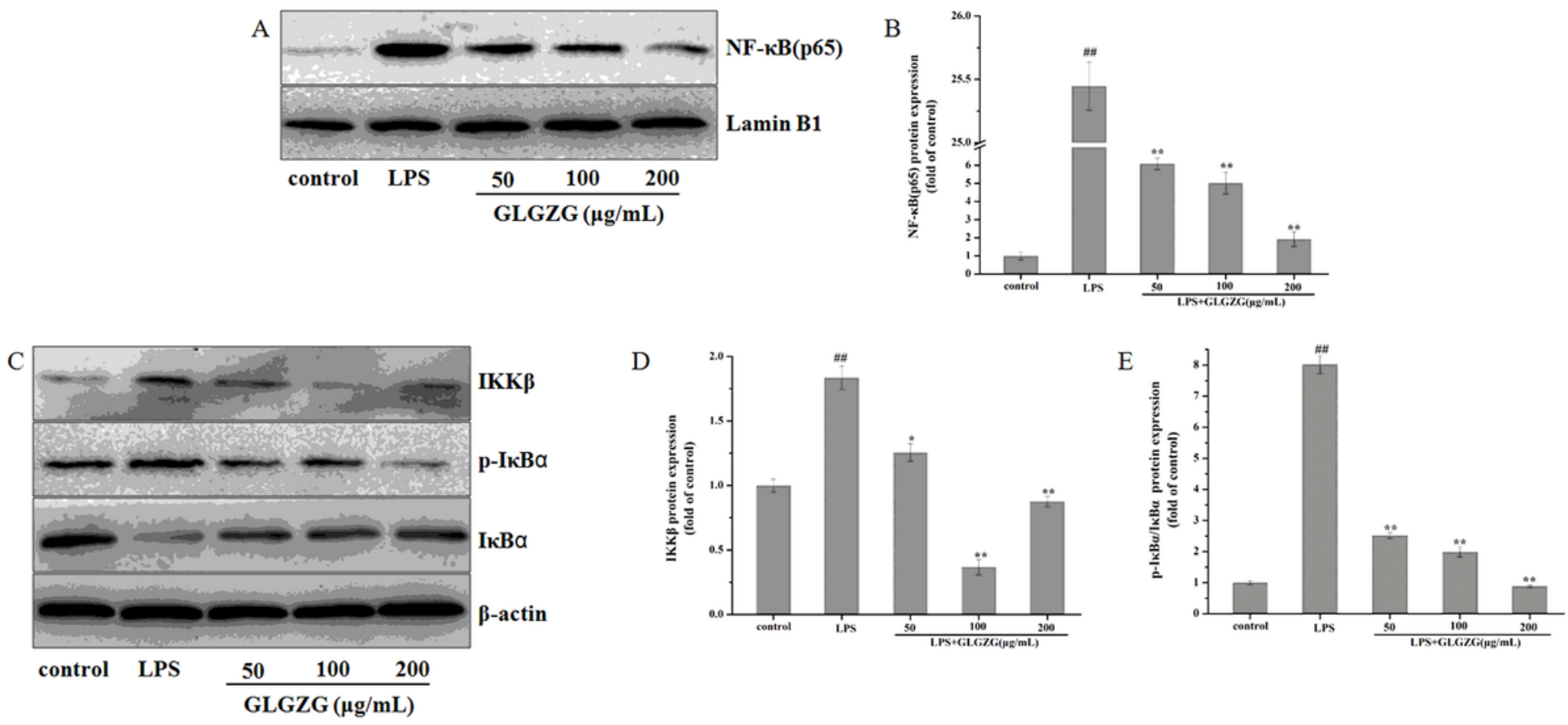

Figure 7

Effect of Gualou Guizhi Granule (GLGZG) on NF-KB signaling in LPS-stimulated BV2. Cells were treated with 50,100 and $200 \mu \mathrm{g} / \mathrm{mL}$ of GLGZG and incubated in the presence of $1 \mu \mathrm{g} / \mathrm{mL}$ LPS for $24 \mathrm{~h}$. After incubation, the protein was extracted from cells, and the levels of NF-KBp65 (A, B) in the nucleus, IkK $3(C$, $D), p-I K B$ and $I K B(C, E)$ were measured by Western blot. Data were shown as means $\pm S D$. \#\#P<0.01 versus the control group. ${ }^{*} \mathrm{P}<0.05$ and ${ }^{*} \mathrm{P}<0.01$ versus the LPS-treated group. 


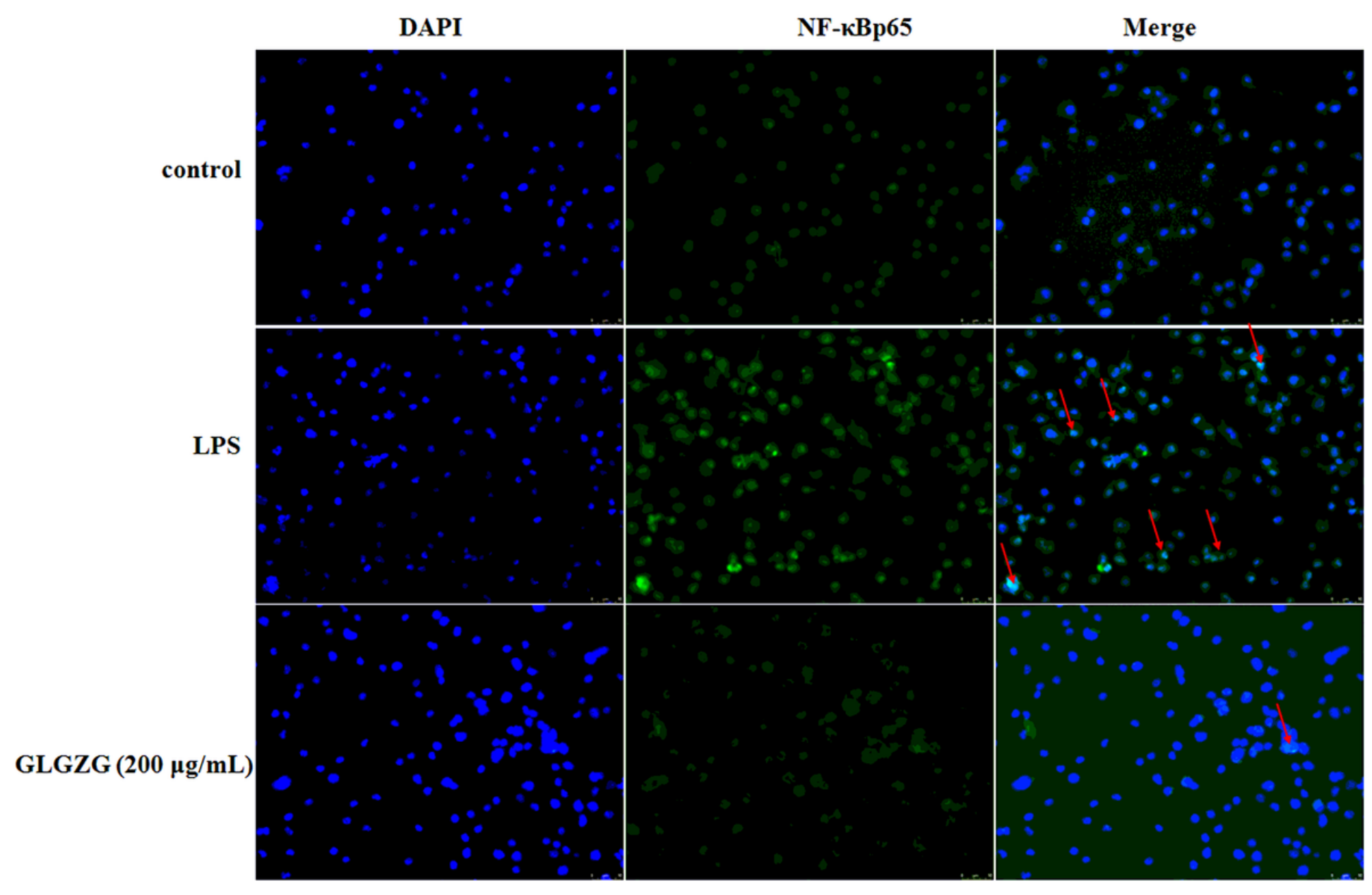

Figure 8

Effect of Gualou Guizhi Granule (GLGZG) on the NF-kB p65 protein localization in LPS-stimulated BV2. Cells were treated with 50,100 and $200 \mu \mathrm{g} / \mathrm{mL}$ of GLGZG and incubated in the presence of $1 \mu \mathrm{g} / \mathrm{mL}$ LPS for $24 \mathrm{~h}$. After incubation, the NF-KB p65 protein localization was detected via immunofluorescence staining. The NF-KB p65 was labelled by FITC-conjugated secondary antibody, and nuclei were counterstained with DAPI. Scale bar, $50 \mu \mathrm{m}$. 


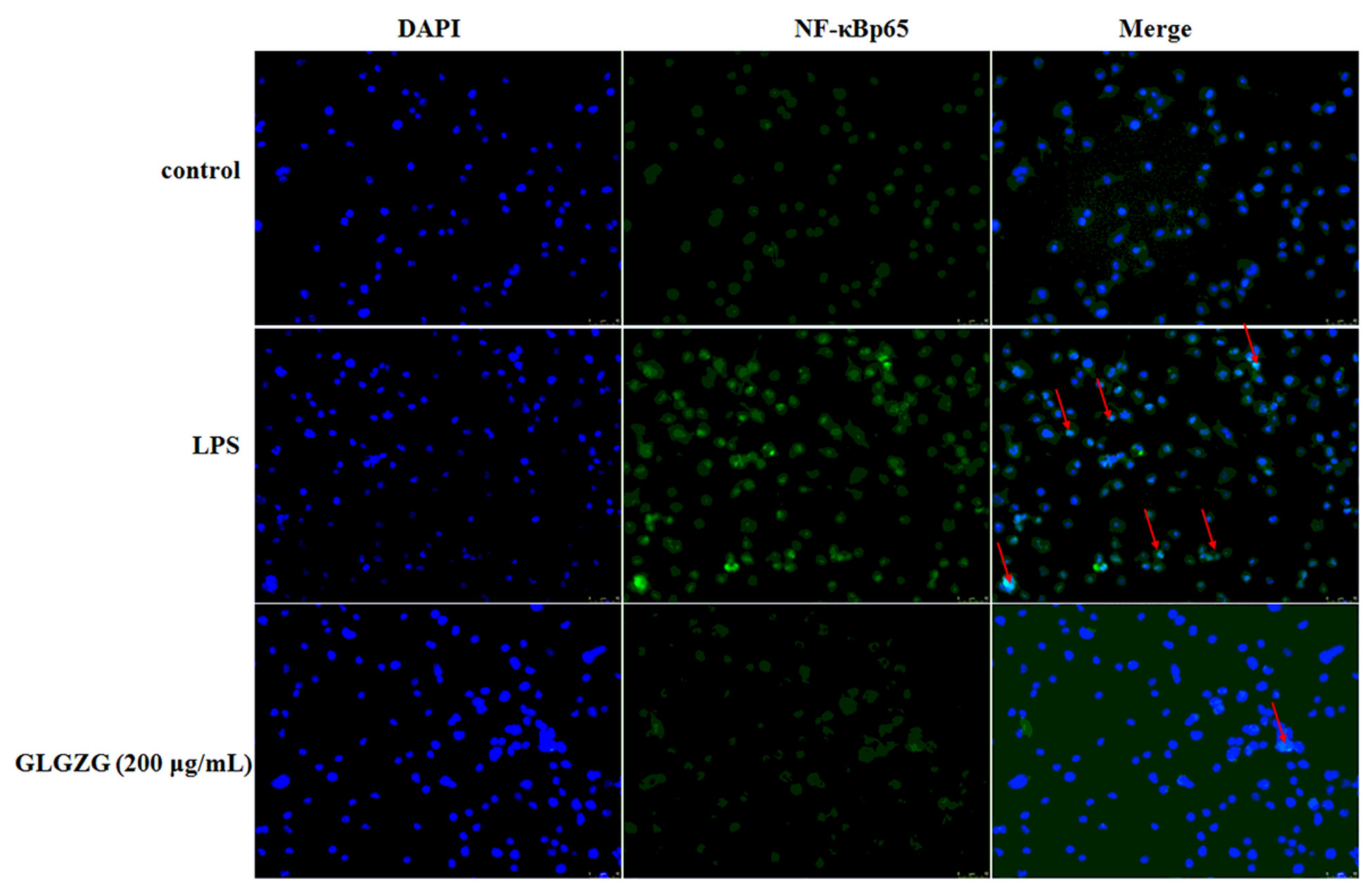

Figure 8

Effect of Gualou Guizhi Granule (GLGZG) on the NF-kB p65 protein localization in LPS-stimulated BV2. Cells were treated with 50,100 and $200 \mu \mathrm{g} / \mathrm{mL}$ of GLGZG and incubated in the presence of $1 \mu \mathrm{g} / \mathrm{mL}$ LPS for $24 \mathrm{~h}$. After incubation, the NF-KB p65 protein localization was detected via immunofluorescence staining. The NF-KB p65 was labelled by FITC-conjugated secondary antibody, and nuclei were counterstained with DAPI. Scale bar, $50 \mu \mathrm{m}$. 


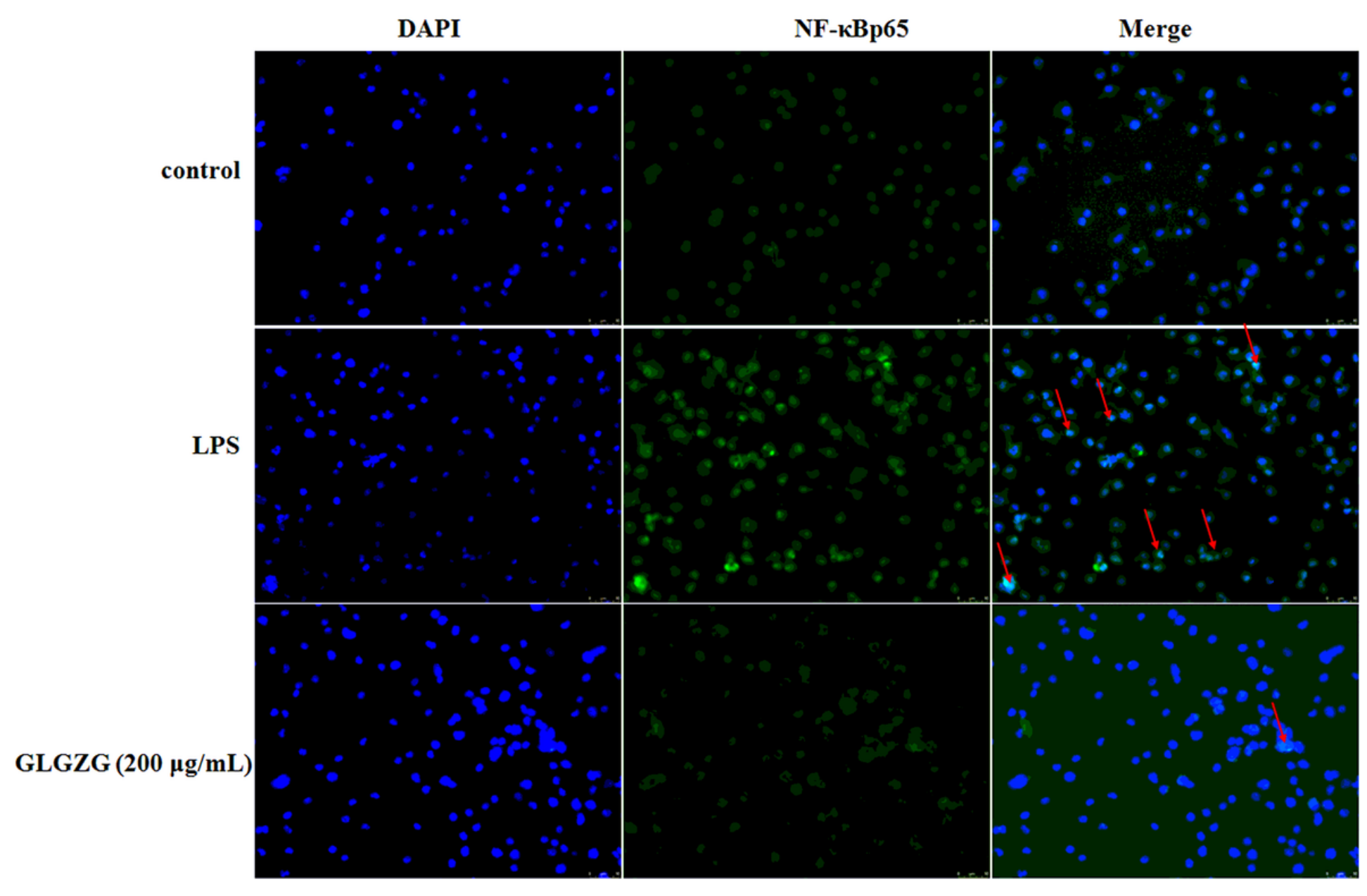

Figure 8

Effect of Gualou Guizhi Granule (GLGZG) on the NF-kB p65 protein localization in LPS-stimulated BV2. Cells were treated with 50,100 and $200 \mu \mathrm{g} / \mathrm{mL}$ of GLGZG and incubated in the presence of $1 \mu \mathrm{g} / \mathrm{mL}$ LPS for $24 \mathrm{~h}$. After incubation, the NF-KB p65 protein localization was detected via immunofluorescence staining. The NF-KB p65 was labelled by FITC-conjugated secondary antibody, and nuclei were counterstained with DAPI. Scale bar, $50 \mu \mathrm{m}$. 


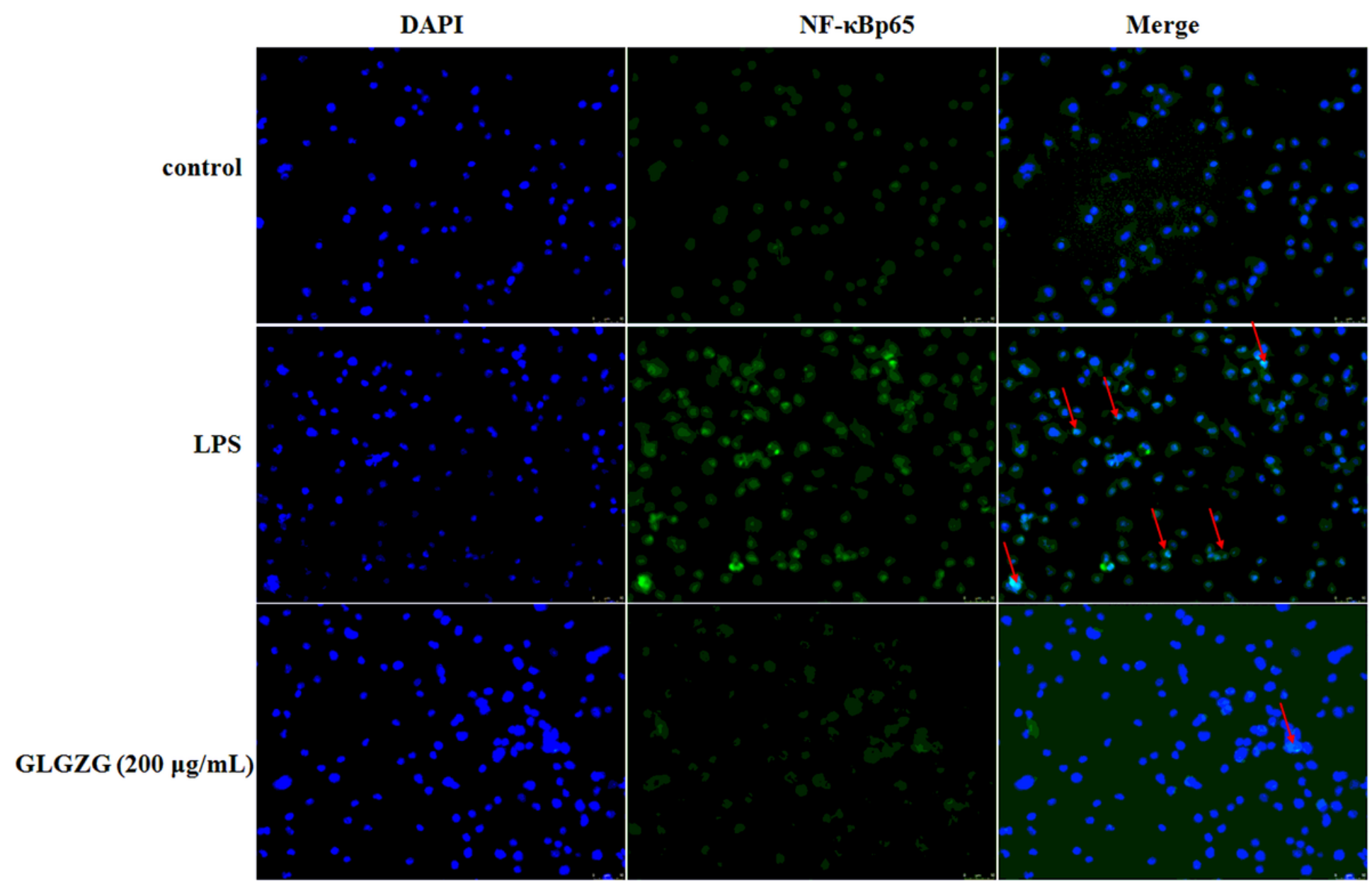

\section{Figure 8}

Effect of Gualou Guizhi Granule (GLGZG) on the NF-KB p65 protein localization in LPS-stimulated BV2. Cells were treated with 50,100 and $200 \mu \mathrm{g} / \mathrm{mL}$ of GLGZG and incubated in the presence of $1 \mu \mathrm{g} / \mathrm{mL}$ LPS for $24 \mathrm{~h}$. After incubation, the NF-KB p65 protein localization was detected via immunofluorescence staining. The NF-kB p65 was labelled by FITC-conjugated secondary antibody, and nuclei were counterstained with DAPI. Scale bar, $50 \mu \mathrm{m}$. 


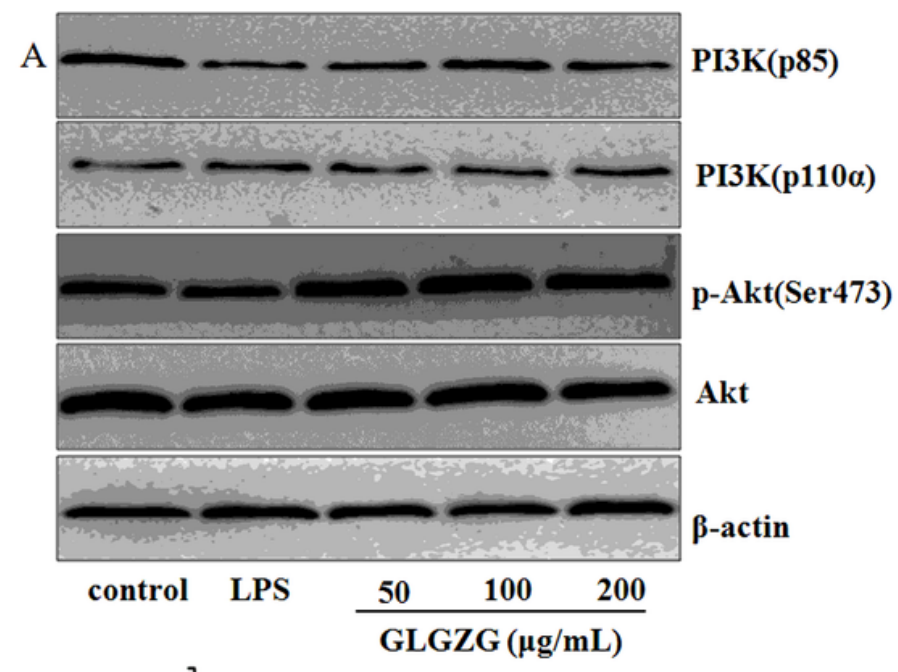

B
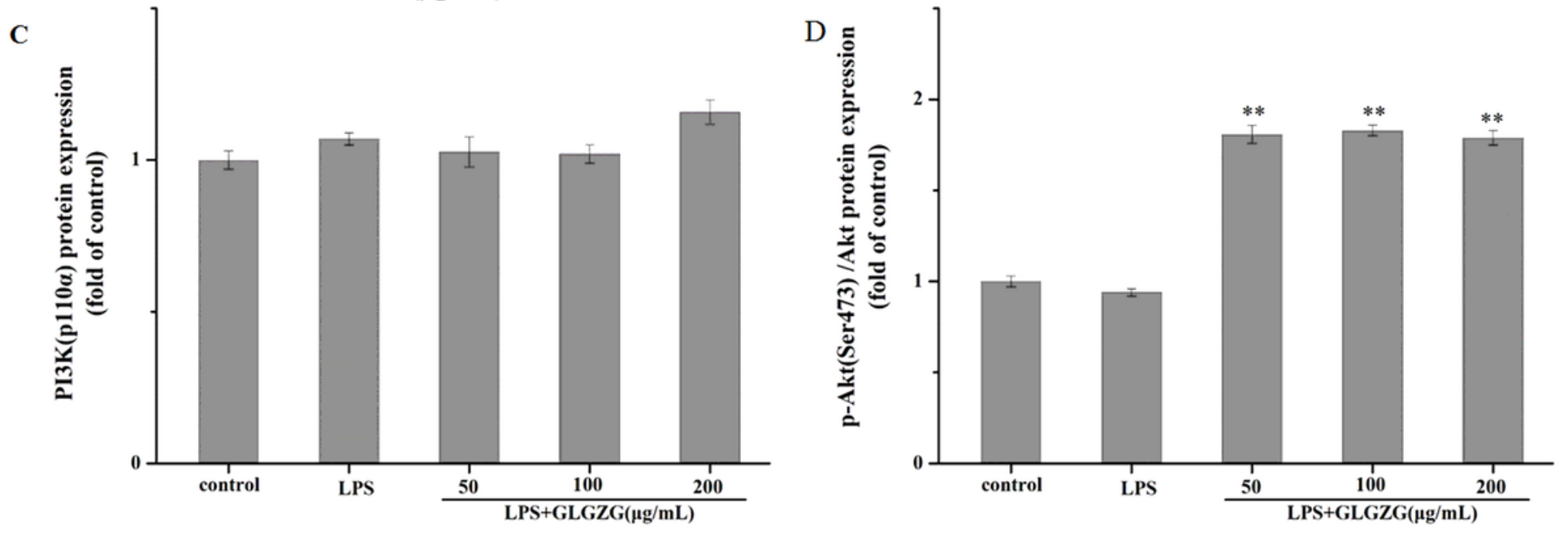

Figure 9

Effect of Gualou Guizhi Granule (GLGZG) on Akt signaling in LPS-stimulated BV2. Cells were treated with 50,100 and $200 \mu \mathrm{g} / \mathrm{mL}$ of GLGZG and incubated in the presence of $1 \mu \mathrm{g} / \mathrm{mL}$ LPS for $24 \mathrm{~h}$. After incubation, the protein was extracted from cells, and the levels of PI3K(p85) (A, B), PI3K(p110a) (A, C), p$\operatorname{Akt}($ Ser473) and Akt $(A, D)$ were measured by Western blot. Data were shown as means $\pm S D$. \#\#P<0.01 versus the control group. ${ }^{*} \mathrm{P}<0.01$ versus the LPS-treated group. 


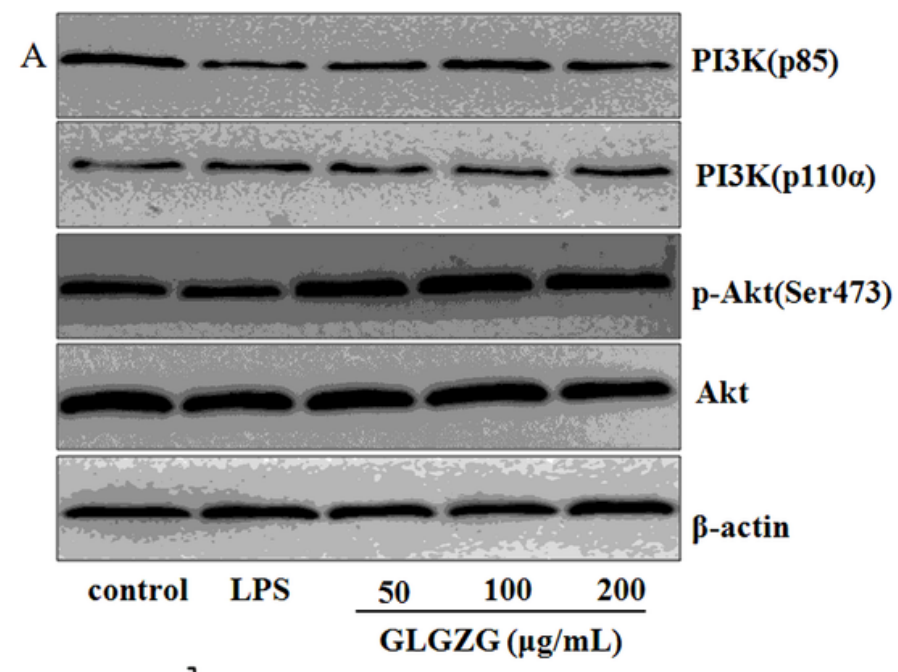

B
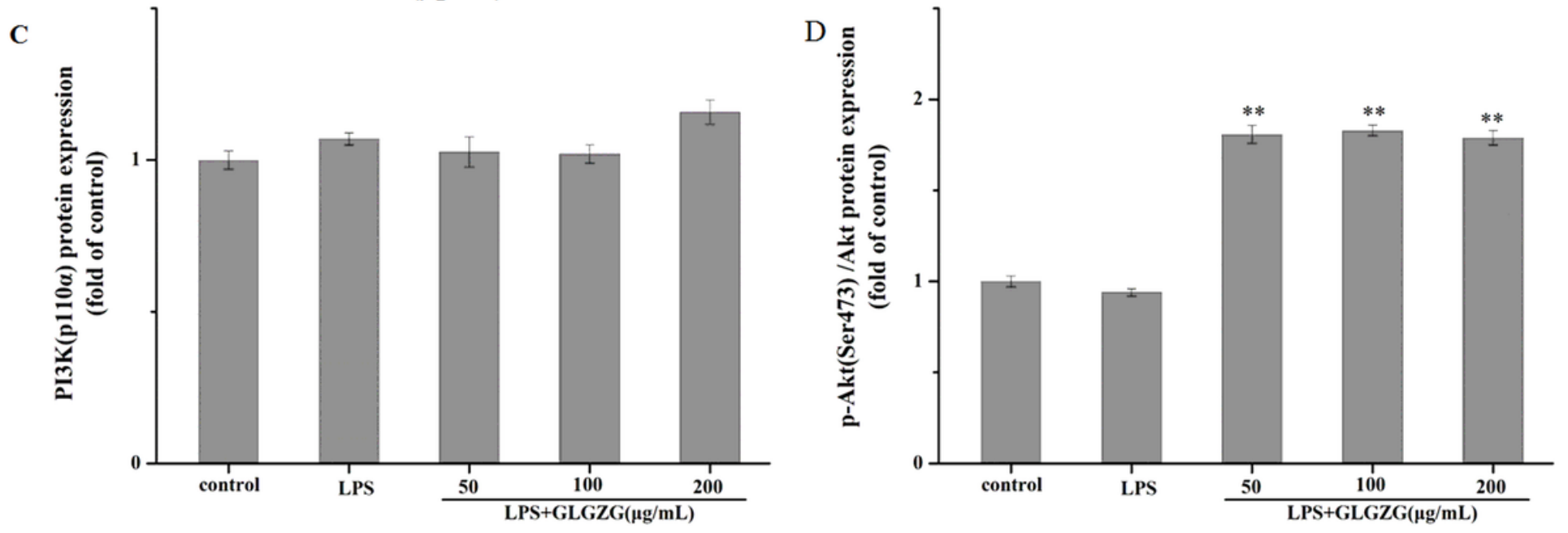

Figure 9

Effect of Gualou Guizhi Granule (GLGZG) on Akt signaling in LPS-stimulated BV2. Cells were treated with 50,100 and $200 \mu \mathrm{g} / \mathrm{mL}$ of GLGZG and incubated in the presence of $1 \mu \mathrm{g} / \mathrm{mL}$ LPS for $24 \mathrm{~h}$. After incubation, the protein was extracted from cells, and the levels of PI3K(p85) (A, B), PI3K(p110a) (A, C), p$\operatorname{Akt}($ Ser473) and Akt $(A, D)$ were measured by Western blot. Data were shown as means $\pm S D$. \#\#P<0.01 versus the control group. ${ }^{*} \mathrm{P}<0.01$ versus the LPS-treated group. 


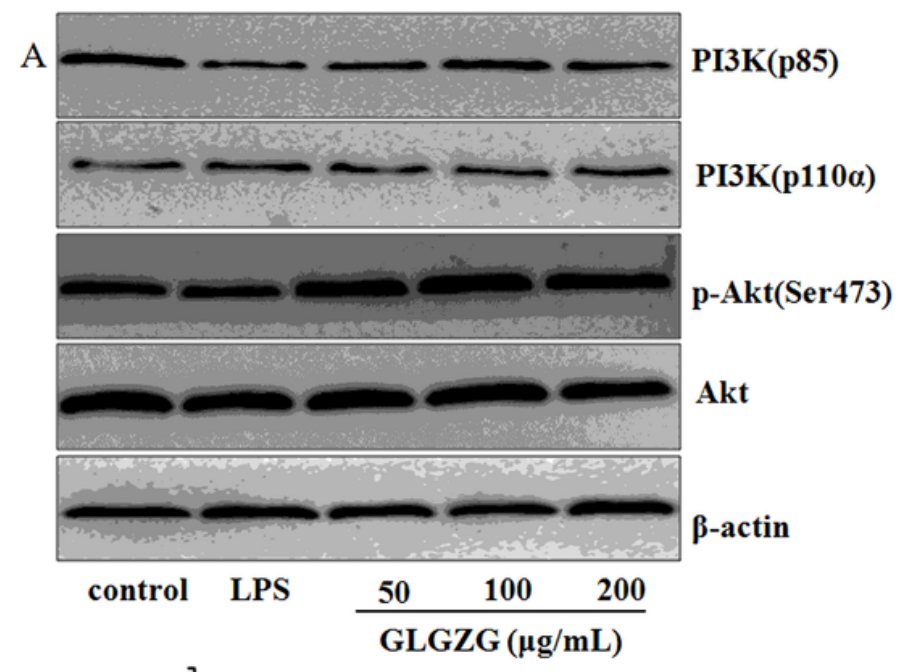

B
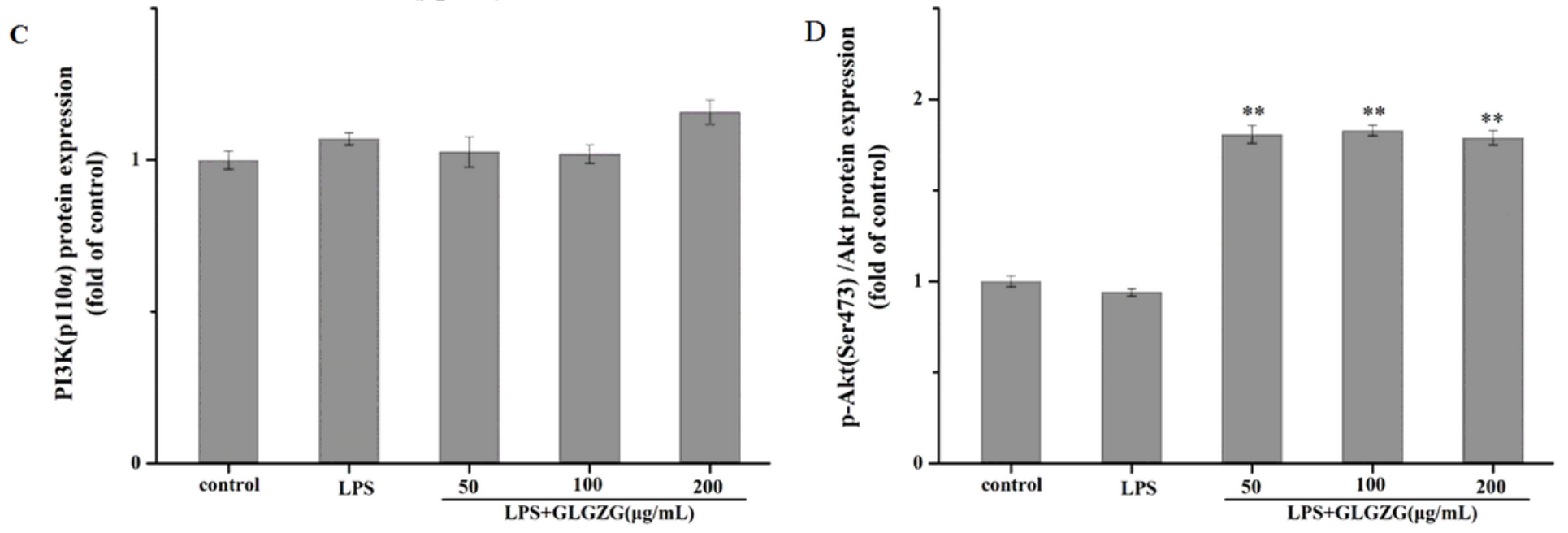

Figure 9

Effect of Gualou Guizhi Granule (GLGZG) on Akt signaling in LPS-stimulated BV2. Cells were treated with 50,100 and $200 \mu \mathrm{g} / \mathrm{mL}$ of GLGZG and incubated in the presence of $1 \mu \mathrm{g} / \mathrm{mL}$ LPS for $24 \mathrm{~h}$. After incubation, the protein was extracted from cells, and the levels of PI3K(p85) (A, B), PI3K(p110a) (A, C), p$\operatorname{Akt}($ Ser473) and Akt $(A, D)$ were measured by Western blot. Data were shown as means $\pm S D$. \#\#P<0.01 versus the control group. ${ }^{*} \mathrm{P}<0.01$ versus the LPS-treated group. 


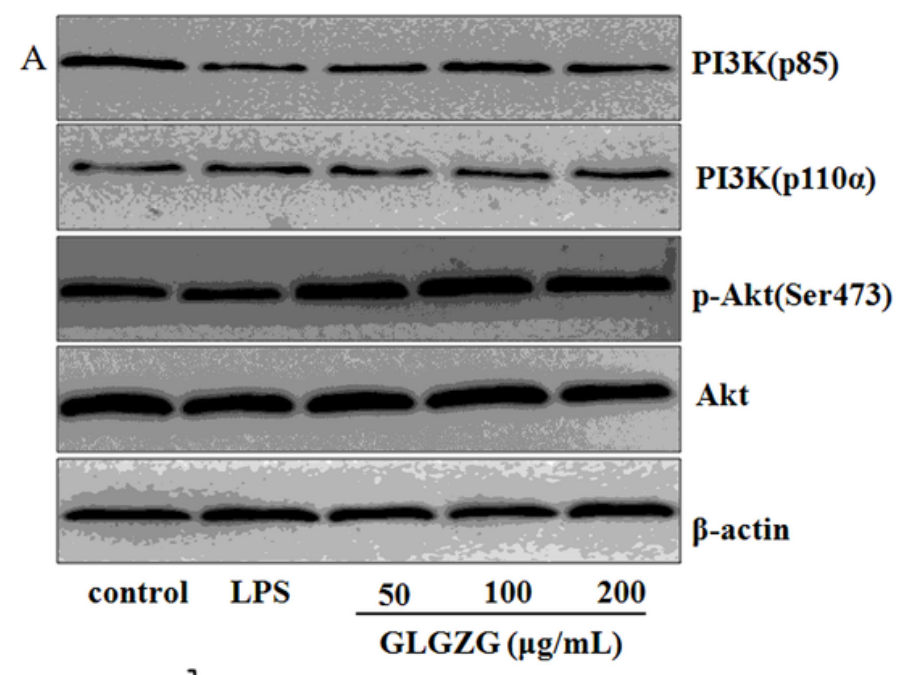

B
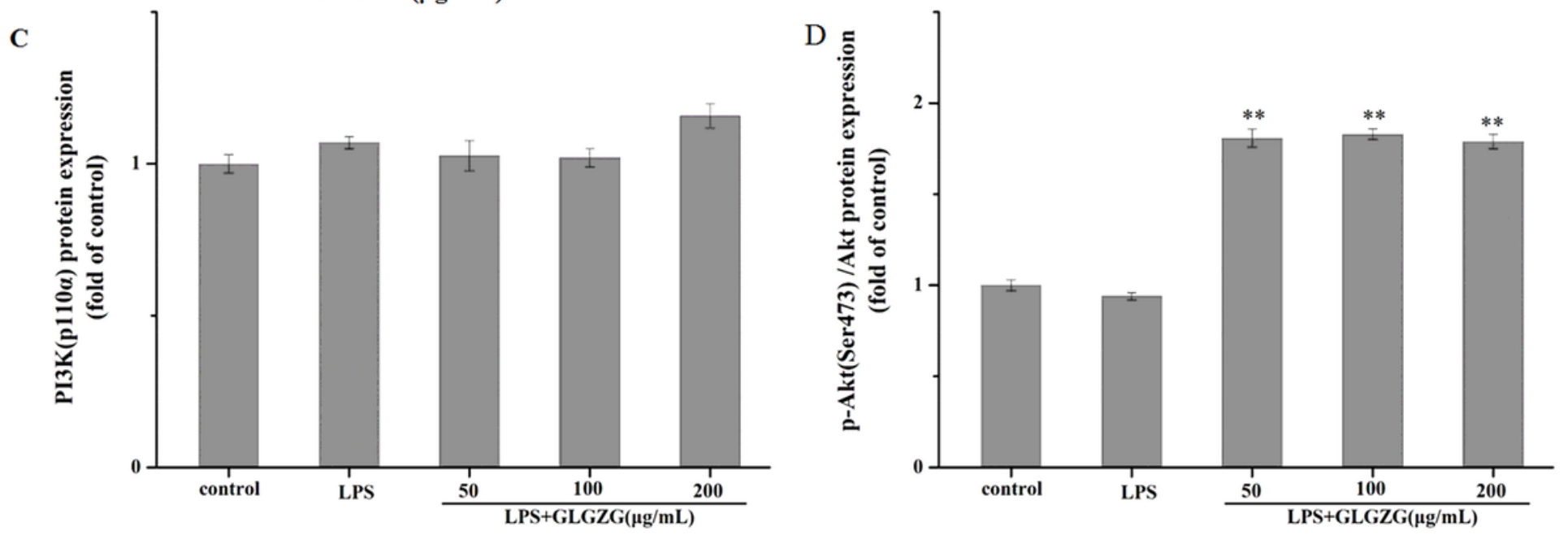

Figure 9

Effect of Gualou Guizhi Granule (GLGZG) on Akt signaling in LPS-stimulated BV2. Cells were treated with 50,100 and $200 \mu \mathrm{g} / \mathrm{mL}$ of GLGZG and incubated in the presence of $1 \mu \mathrm{g} / \mathrm{mL}$ LPS for $24 \mathrm{~h}$. After incubation, the protein was extracted from cells, and the levels of PI3K(p85) (A, B), PI3K(p110a) (A, C), p$\operatorname{Akt}($ Ser473) and Akt $(A, D)$ were measured by Western blot. Data were shown as means $\pm S D$. \#\#P<0.01 versus the control group. ${ }^{*} \mathrm{P}<0.01$ versus the LPS-treated group. 

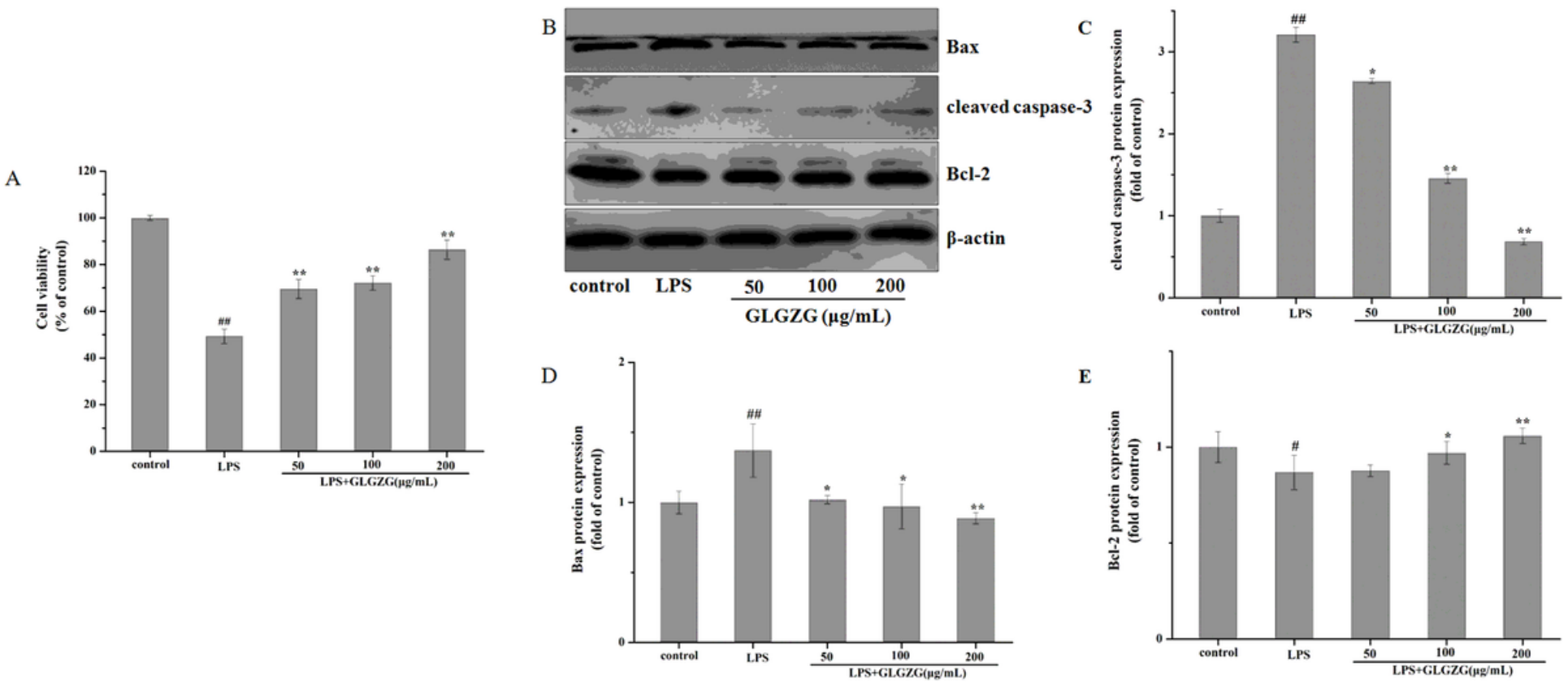

Figure 10

Effect of Gualou Guizhi Granule (GLGZG) on the neurotoxicity of LPS-activated BV2 microglia cells in HT22 cells. BV2 cells were activated by LPS. Conditioned media from control, LPS-activated, and LPS + GLGZG (50, 100 and $200 \mu \mathrm{g} / \mathrm{mL}$ )-treated cells were transferred to neuronal cell line, HT-22 cells. HT-22 cells were treated with these conditioned media, supernatant was harvested for cell viability, and cells were harvested for cleaved-caspase3, Bax and Bcl-2 protein analysis. (A) Cell viability was determined by CellTiter $96 \AA$ AQ ueous one solution. Results were expressed as percentages relative to the control group. Data were shown as means \pm SD of six independent experiments in triplicate. (B-E) the protein was extracted from cells, and the levels of cleaved-caspase3, Bax and Bcl-2 were measured by Western blot. Data were shown as means $\pm S D$. $\# P<0.05$ and $\# \# P<0.01$ versus the control group. ${ }^{*} P<0.05$ and $* * P<0.01$ versus the LPS-treated group.
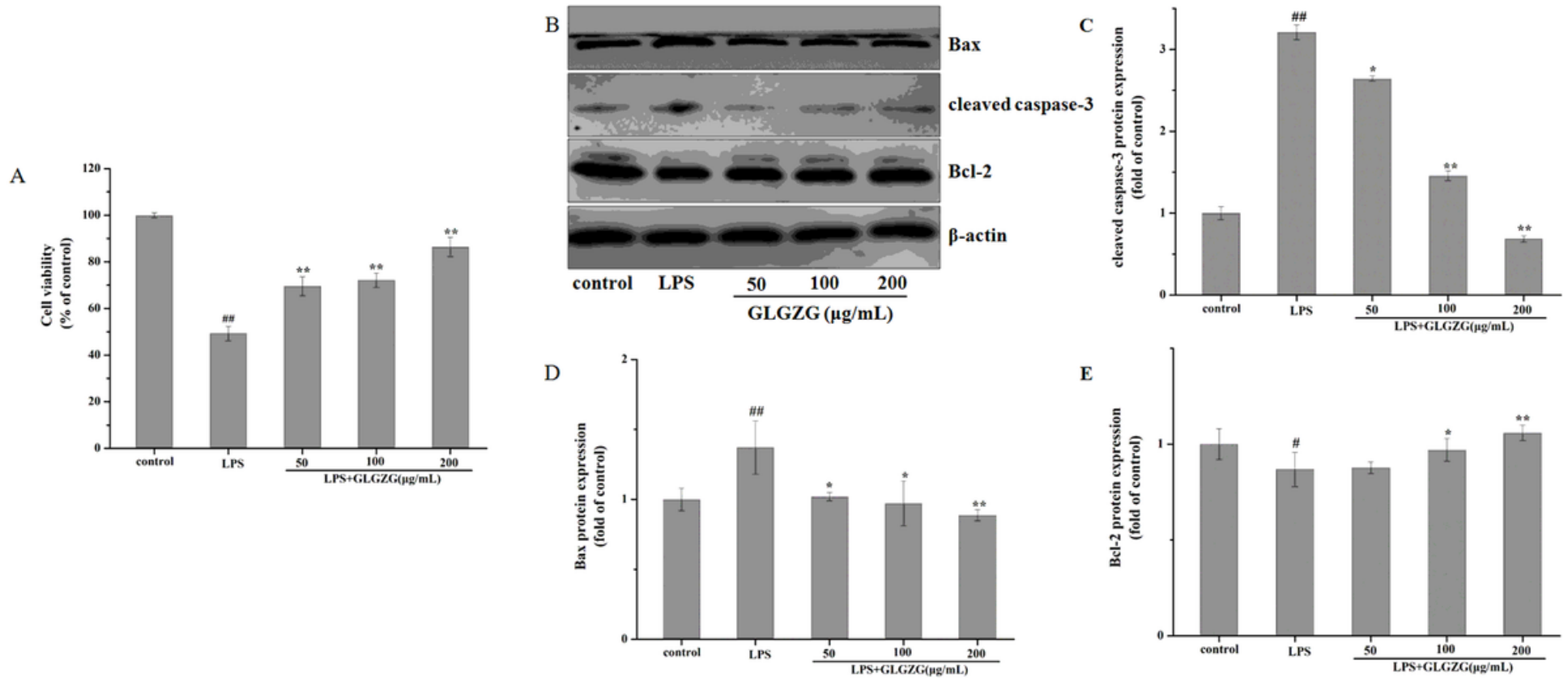
Effect of Gualou Guizhi Granule (GLGZG) on the neurotoxicity of LPS-activated BV2 microglia cells in HT22 cells. BV2 cells were activated by LPS. Conditioned media from control, LPS-activated, and LPS + GLGZG (50, 100 and $200 \mu \mathrm{g} / \mathrm{mL})$-treated cells were transferred to neuronal cell line, HT-22 cells. HT-22 cells were treated with these conditioned media, supernatant was harvested for cell viability, and cells were harvested for cleaved-caspase3, Bax and Bcl-2 protein analysis. (A) Cell viability was determined by CellT iter $96 \AA A Q$ ueous one solution. Results were expressed as percentages relative to the control group. Data were shown as means $\pm S D$ of six independent experiments in triplicate. (B-E) the protein was extracted from cells, and the levels of cleaved-caspase3, Bax and Bcl-2 were measured by Western blot. Data were shown as means $\pm S D$. $\# P<0.05$ and $\# \# P<0.01$ versus the control group. ${ }^{*} P<0.05$ and ${ }^{* * P}<0.01$ versus the LPS-treated group.

A

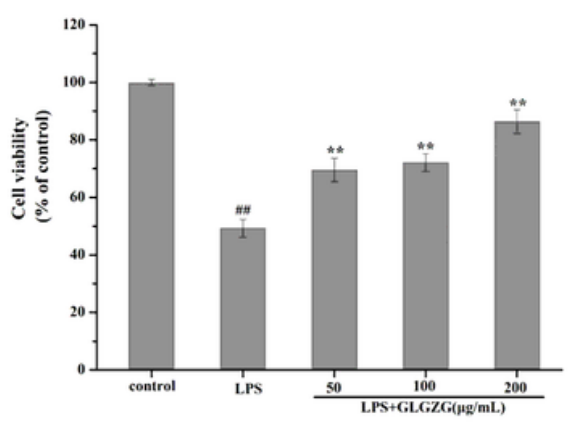

в

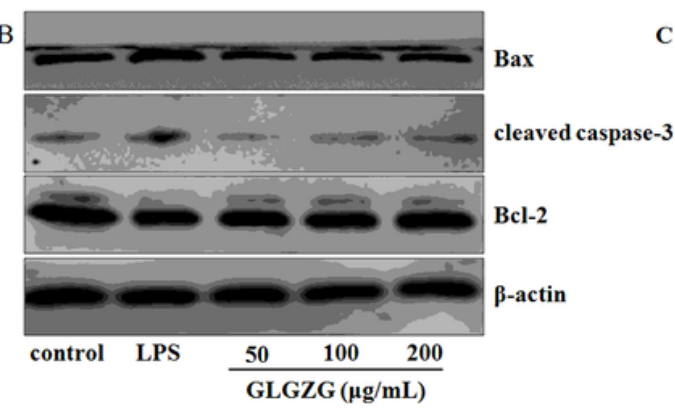

D

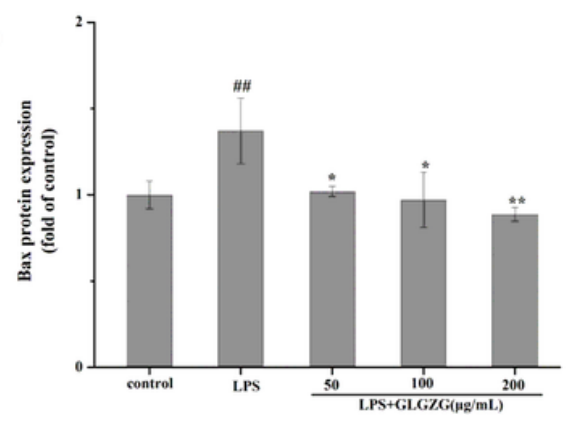

C

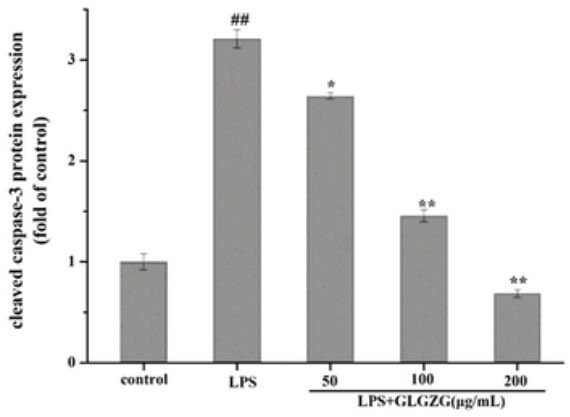

E

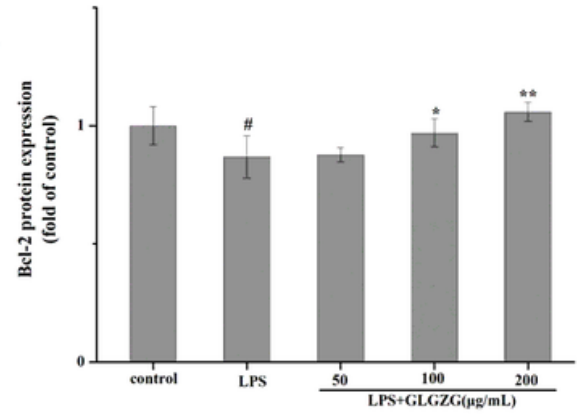

Figure 10

Effect of Gualou Guizhi Granule (GLGZG) on the neurotoxicity of LPS-activated BV2 microglia cells in HT22 cells. BV2 cells were activated by LPS. Conditioned media from control, LPS-activated, and LPS + GLGZG (50, 100 and $200 \mu \mathrm{g} / \mathrm{mL}$ )-treated cells were transferred to neuronal cell line, HT-22 cells. HT-22 cells were treated with these conditioned media, supernatant was harvested for cell viability, and cells were harvested for cleaved-caspase3, Bax and Bcl-2 protein analysis. (A) Cell viability was determined by CellTiter $96 \AA$ AQ ueous one solution. Results were expressed as percentages relative to the control group. Data were shown as means \pm SD of six independent experiments in triplicate. (B-E) the protein was extracted from cells, and the levels of cleaved-caspase3, Bax and $\mathrm{Bcl}-2$ were measured by Western blot. Data were shown as means $\pm S D$. \#P<0.05 and \#\#P<0.01 versus the control group. ${ }^{*} P<0.05$ and ${ }^{\star *} P<0.01$ versus the LPS-treated group. 

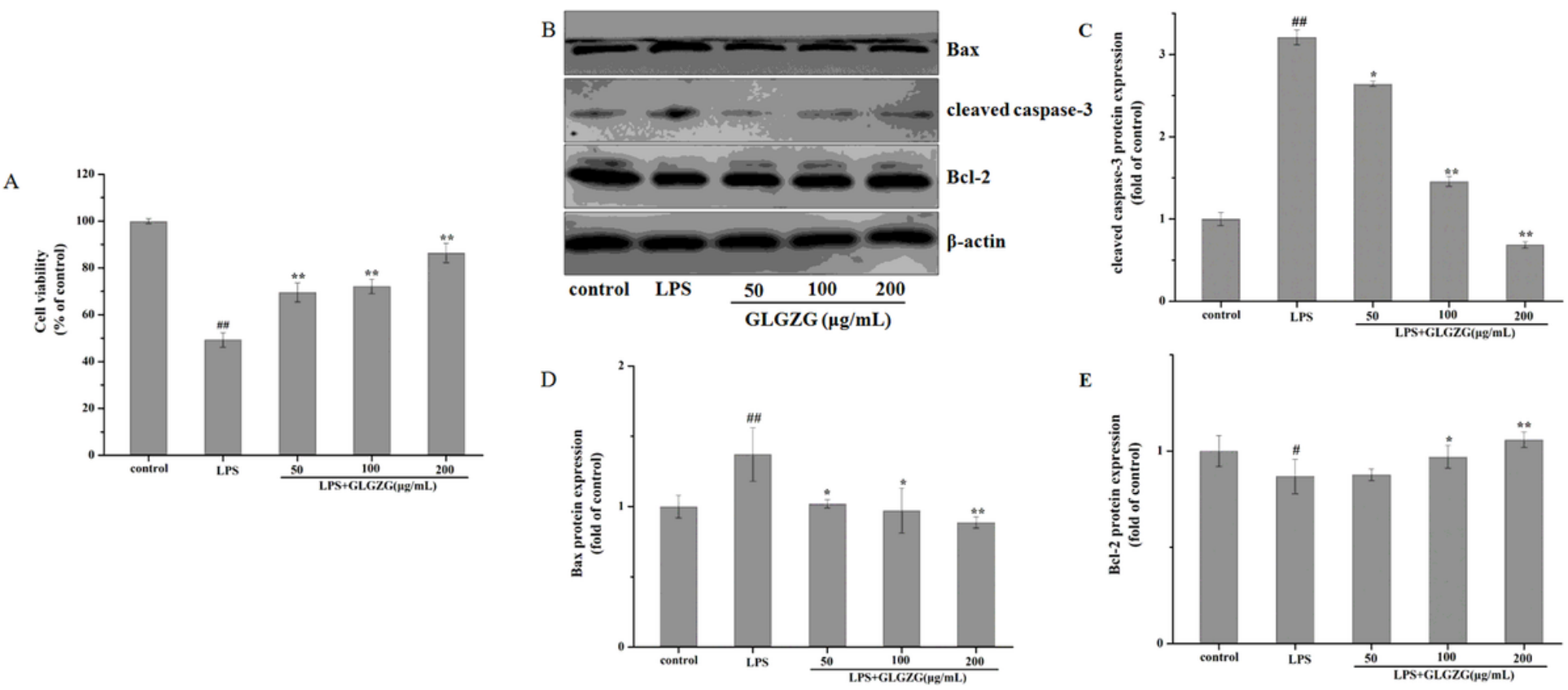

Figure 10

Effect of Gualou Guizhi Granule (GLGZG) on the neurotoxicity of LPS-activated BV2 microglia cells in HT22 cells. BV2 cells were activated by LPS. Conditioned media from control, LPS-activated, and LPS + GLGZG (50, 100 and $200 \mu \mathrm{g} / \mathrm{mL}$ )-treated cells were transferred to neuronal cell line, HT-22 cells. HT-22 cells were treated with these conditioned media, supernatant was harvested for cell viability, and cells were harvested for cleaved-caspase3, Bax and Bcl-2 protein analysis. (A) Cell viability was determined by CellT iter $96 \AA$ AQ ueous one solution. Results were expressed as percentages relative to the control group. Data were shown as means \pm SD of six independent experiments in triplicate. (B-E) the protein was extracted from cells, and the levels of cleaved-caspase3, Bax and Bcl-2 were measured by Western blot. Data were shown as means \pm SD. $\# P<0.05$ and \#\#P<0.01 versus the control group. ${ }^{*} P<0.05$ and ${ }^{*} P<<0.01$ versus the LPS-treated group.

\section{Supplementary Files}

This is a list of supplementary files associated with this preprint. Click to download.

- A.tif

- A.tif

- A.tif

- A.tif 